\title{
Quantization for an elliptic equation with critical exponential growth on compact Riemannian surface without boundary
}

\author{
Yunyan Yang \\ Department of Mathematics, Renmin University of China, Beijing 100872, P. R. China
}

\begin{abstract}
In this paper, using blow-up analysis, we prove a quantization result for an elliptic equation with critical exponential growth on compact Riemannian surface without boundary. Similar results for Euclidean space were obtained by Adimurthi-Struwe [2], Druet [6], Lamm-Robert-Struwe [8], Martinazzi [9], Martinazzi-Struwe [10], and Struwe [13] respectively.
\end{abstract}

Key words: Quantization, Multi-bubble analysis, Trudinger-Moser inequality 2010 MSC: 58J05

\section{Contents}

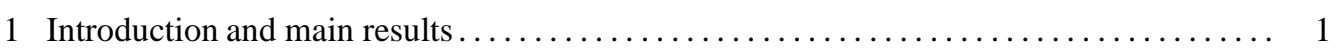

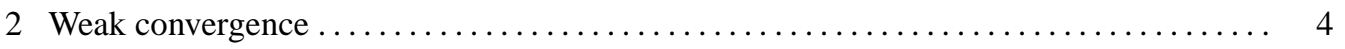

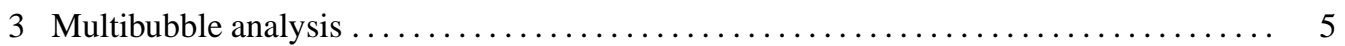

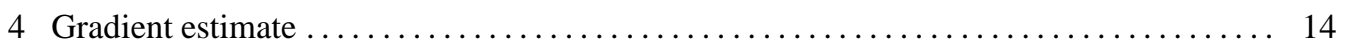

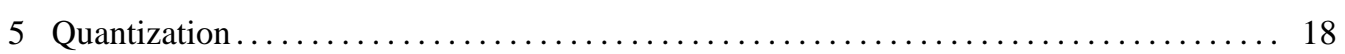

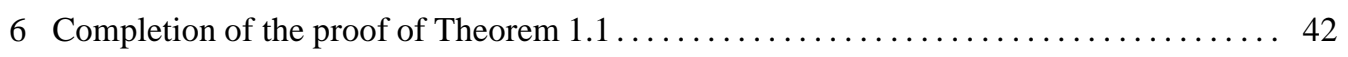

\section{Introduction and main results}

Let $(\Sigma, g)$ be a compact Riemannian surface without boundary, $W^{1,2}(\Sigma, \mathbb{R})$ be the usual Sobolev space, namely the completion of $C^{\infty}(\Sigma, \mathbb{R})$ under the norm

$$
\|u\|_{W^{1,2}(\Sigma, \mathbb{R})}=\left(\int_{\Sigma}\left(\left|\nabla_{g} u\right|^{2}+u^{2}\right) d v_{g}\right)^{1 / 2},
$$

where $\nabla_{g} u$ denotes the gradient of $u$ and $d v_{g}$ denotes the volume element with respect to the Riemannian metric $g$. Let $f_{k}: \Sigma \times[0, \infty) \rightarrow \mathbb{R}$ be a sequence of functions satisfying the following hypotheses:

Email address: yunyanyang@ruc.edu.cn (Yunyan Yang) 
(H1) $f_{k}(x, 0)=0$, and $f_{k}(x, t)>0$ for all $k$, all $x \in \Sigma$, and all $t>0$;

(H2) $f_{k} \in C^{2}(\Sigma \times[0,+\infty))$ for each $k$ and $f_{k} \rightarrow f_{\infty}$ in $C_{\mathrm{loc}}^{1}(\Sigma \times[0,+\infty))$ as $k \rightarrow \infty$;

(H3) for any $v>0$, there exists a constant $C_{v}>0$ such that for all $k$, all $x \in \Sigma$, and all $t>0$,

$$
F_{k}(x, t) \leq v t f_{k}(x, t)+C_{v}
$$

where

$$
F_{k}(x, t)=\int_{0}^{t} f_{k}(x, s) d s
$$

is the primitive of $f_{k}(x, t)$;

(H4) $f_{k}^{\prime}(x, t) /\left(t f_{k}(x, t)\right) \rightarrow 2$ as $t \rightarrow+\infty$ uniformly in $k \in \mathbb{N}$ and in $x \in \Sigma$, where $f_{k}^{\prime}$ is the derivative of $f_{k}$ with respect to $t$, moreover there exists a constant $C$ such that $\left|\nabla_{g} f_{k}(x, t)\right| \leq$ $C\left(1+f_{k}(x, t)\right)$ for all $(x, t) \in \Sigma \times \mathbb{R}$;

(H5) there exist $\psi$, a continuous function with $\psi(0)=0, t_{0}>0$, and $k_{0}>0$, such that

$$
\left|f_{k}(x, t) / f_{k}(y, t)-1\right| \leq \psi\left(d_{g}(x, y)\right)
$$

for all $t \geq t_{0}$, all $k>k_{0}$, and all $x, y \in \Sigma$, where $d_{g}(\cdot, \cdot)$ denotes the geodesic distance between two points of $\Sigma$.

By (H4) we have $f_{k}(x, t)=f_{k}\left(x, t_{0}\right) e^{(1+o(1))\left(t^{2}-t_{0}^{2}\right)}$ for any given $t_{0}>0$, where $o(1) \rightarrow 0$ as $t \rightarrow \infty$ uniformly in $x \in \Sigma$. In view of the Trudinger-Moser embedding [7, 11, 12, 14], we say that $f_{k}(x, t)$ is of critical exponential growth with respect to $t$. A typical example satisfying (H1)-(H5) is

$$
f_{k}(x, t)=\lambda_{k} t e^{t^{2}}
$$

where $\lambda_{k}$ is a sequence of positive real numbers such that $\lambda_{k} \rightarrow \lambda_{\infty}$ as $k \rightarrow \infty$. Suppose that for each $k \in \mathbb{N}$ we have a smooth function $u_{k} \geq 0$ satisfying the equation

$$
\Delta_{g} u_{k}+\tau_{k} u_{k}=f_{k}\left(x, u_{k}\right) \text { in } \Sigma,
$$

where $\Delta_{g}$ is the Laplace-Beltrami operator, $\tau_{k}$ is a sequence of smooth functions such that

$$
\tau_{k} \rightarrow \tau_{\infty} \text { in } C^{0}(\Sigma, \mathbb{R}), \tau_{\infty}(x)>0 \text { for all } x \in \Sigma .
$$

Clearly $u_{k}$ is a critical point of the functional

$$
J_{k}(u)=\frac{1}{2} \int_{\Sigma}\left(\left|\nabla_{g} u\right|^{2}+\tau_{k} u^{2}\right) d v_{g}-\int_{\Sigma} F_{k}(x, u) d v_{g}
$$

on the Sobolev space $W^{1,2}(\Sigma, \mathbb{R})$. The existence of nonnegative solutions to equation (1.2) in case that $\tau_{k}$ is a positive real number was studied by Zhao and the author [16] by using variational methods. More explicitly, assuming that $\lambda_{\tau}=\lambda_{\tau}(\Sigma)$ is the first eigenvalue of the operator $\Delta_{g}+\tau$, where $\tau>0$ is a constant, we proved that the equation $\Delta_{g} u+\tau u=\lambda u e^{u^{2}}$ has a nonnegative solution if $\lambda<\lambda_{\tau}$. The aim of this paper is to study the quantization problem for equation (1.2). Precisely we shall prove the following result.

Theorem 1.1 Let $(\Sigma, g)$ be a compact Riemannian surface without boundary. Suppose that $u_{k} \geq 0$ is a sequence of smooth solutions to equation (1.2), where $\tau_{k}$ is a sequence of smooth functions 
satisfying (1.3), and $f_{k}$ is a sequence of functions satisfying (H1)-(H5). Let $J_{k}$ be as in (1.4). If $J_{k}\left(u_{k}\right) \rightarrow \beta$ as $k \rightarrow \infty$ for some $\beta \in \mathbb{R}$, then there exists a nonnegative solution $u_{\infty} \in C^{1}(\Sigma, \mathbb{R})$ of the equation

$$
\Delta_{g} u_{\infty}+\tau_{\infty} u_{\infty}=f_{\infty}\left(x, u_{\infty}(x)\right) \text { in } \Sigma,
$$

and there exists $N \in \mathbb{N}$ such that $J_{k}\left(u_{k}\right)=J_{\infty}\left(u_{\infty}\right)+2 \pi N+o(1)$, where $o(1) \rightarrow 0$ as $k \rightarrow \infty$. Here $J_{\infty}$ is also as in (1.4), where $\tau_{k}, F_{k}$ are replaced by $\tau_{\infty}$ and $F_{\infty}$ respectively. If $N=0, u_{k} \rightarrow u_{\infty}$ strongly in $W^{1,2}(\Sigma, \mathbb{R})$ and in fact in $C^{1}(\Sigma, \mathbb{R})$.

Several works were devoted to prove analogues of Theorem 1.1. In [2], Adimurthi and Struwe considered a sequence of solutions $u_{k}$ to the equation

$$
\left\{\begin{array}{l}
-\Delta_{\mathbb{R}^{2}} u_{k}=f_{k}\left(x, u_{k}\right) \text { in } \Omega \subset \mathbb{R}^{2} \\
u_{k}>0 \text { in } \Omega, u_{k}=0 \text { on } \partial \Omega,
\end{array}\right.
$$

where $f_{k}(x, t)=t e^{\varphi_{k}(t)}, 0 \leq \varphi_{k}^{\prime \prime}(t) \leq 2$ for $t \geq t_{0}$ and $\varphi_{k}^{\prime}(t) / t \rightarrow 2$ as $t \rightarrow \infty$ uniformly in $k$. Such a sequence of functions $f_{k}$ satisfies (H1)-(H5) in case that the Riemannian surface $(\Sigma, g)$ is replaced by a smooth bounded domain of $\mathbb{R}^{2}$. Assuming that

$$
J_{k}\left(u_{k}\right)=\frac{1}{2} \int_{\Omega}\left|\nabla_{\mathbb{R}^{2}} u_{k}\right|^{2} d x-\int_{\Omega} F_{k}\left(x, u_{k}\right) d x \rightarrow \beta
$$

for $0 \leq \beta<4 \pi$ and that the limit equation does not admit any positive solution with energy less than $2 \pi$, they proved that either $u_{k} \rightarrow u_{\infty}$ strongly in $W_{0}^{1,2}(\Omega)$ and $u_{\infty}$ has energy $\beta$, or $u_{k} \rightarrow 0$ weakly in $W_{0}^{1,2}(\Omega)$ and $u_{k}$ develops one blow-up point carrying the energy $2 \pi$. This quantization result was surprisingly refined by Druet [6] to the case of all $\beta \in \mathbb{R}$ and general nonlinearities of uniform critical growth, analogous to that of the current paper. (Blow-up analysis for equation (1.6) with similar nonlinearity was also considered by Adimuthi and Druet [1].) The key point in [6] is the gradient estimate ([6], Proposition 2), through which Druet studied the energy of $\varphi_{k}$, the spherical average of $u_{k}$ with respect to blow-up points, instead of $u_{k}$ itself. Thus he transformed the quantization problem for $u_{k}$ to the quantization problem for $\varphi_{k}$, which depends only on analysis on certain ordinary differential equation and is comparatively easy to be handled. Shortly after, using similar idea, Struwe [13] succeeded to get a quantization result for a forth order elliptic equation

$$
\left\{\begin{array}{l}
-\Delta_{\mathbb{R}^{4}}^{2} u_{k}=\lambda_{k} u_{k} e^{2 u_{k}^{2}} \text { in } \Omega \subset \mathbb{R}^{4} \\
u_{k}>0 \text { in } \Omega, \quad u_{k}=\Delta_{\mathbb{R}^{4}} u_{k}=0 \text { on } \partial \Omega,
\end{array}\right.
$$

where $0<\lambda_{k} \rightarrow 0$ as $k \rightarrow \infty$, and $u_{k} \rightarrow 0$ weakly in $W^{2,2}(\Omega)$. Also Lamm, Robert and Struwe [8] proved a quantization result for the evolution of equation (1.6), where $f_{k}$ is as in (1.1). A recent inspiring work of Martinazzi and Struwe [10] states the following: Let $\Omega \subset \mathbb{R}^{2 m}$ be a smooth bounded domain, $u_{k}$ be a sequence of positive solutions to the equation $-\Delta_{\mathbb{R}^{2}} u_{k}=\lambda_{k} u_{k} e^{m u_{k}^{2}}$ subject to Dirichlet boundary conditions, where $0<\lambda_{k} \rightarrow 0$ and $u_{k} \rightarrow 0$ weakly in $W^{m, 2}(\Omega)$. Assuming $\Lambda=\lim _{k \rightarrow \infty} \int_{\Omega} u_{k}\left(-\Delta_{\mathbb{R}^{2 m}}\right)^{m} u_{k} d x<\infty$, they proved that $\Lambda$ is an integer multiple of $\Lambda_{1}=(2 m-1) ! \operatorname{vol}\left(\mathbb{S}^{2 m}\right)$, the total $Q$-curvature of the standard $2 m$-dimensional sphere. In view of the Trudinger-Moser embedding for the space $W_{0}^{1, n}(\Omega)$, where $n \geq 3$ and $\Omega \subset \mathbb{R}^{n}$ is a smooth 
bounded domain, one may ask how about the equation

$$
\left\{\begin{array}{l}
-\Delta_{n} u_{k}=\lambda_{k} u_{k}^{\frac{1}{n-1}} e^{u_{k}^{\frac{n}{n-1}}} \text { in } \Omega \\
u_{k} \geq 0 \text { in } \Omega, u_{k}=0 \text { on } \partial \Omega .
\end{array}\right.
$$

Up to now only an energy inequality has been obtained by Adimurthi and the author [3]. Concerning the quantization for equation (1.7), we have a long way to go. For other works related to this kind of quantization problems we refer the reader to [10, 13] and the references therein.

For the proof of Theorem 1.1, we follow the lines of [6, 8, 10, 13]. Firstly we use a pointwise estimate on $u_{k}$ to find all separate blow-up points. Specifically we need to deal carefully with the term $\tau_{k} u_{k}$, which does not appear in the Euclidean case. Secondly we establish a gradient estimate for $u_{k}$. This permits us to compare $u_{k}$ with its spherical average with respect to blow-up points. Finally we get the quantization result, where we should deal with the extra term $\tau_{k} u_{k}$ again. For calculations near blow-up points we prefer to choose isothermal coordinates instead of normal coordinates. The advantage of such coordinates is that both the Laplace-Beltrami operator $\Delta_{g}$ and the gradient operator $\nabla_{g}$ have simple expressions.

The remaining part of this paper is organized as follows. In the next section we prove a simple property of the weak convergence of $u_{k}$. In Section 3, we locate the blow-up points of $u_{k}$ and describe the asymptotic behavior of $u_{k}$ near those points. In Section 4 we derive a gradient estimate on $u_{k}$. We shall prove quantization results for $u_{k}$ near the blow-up points in Section 5 , and complete the proof of Theorem 1.1 in Section 6.

Throughout this paper we often denote various constants independent of $k$ by the same $C$. In addition, we do not distinguish between sequence and subsequence or points and sequence sometimes. The reader can easily recognize it from the context.

\section{Weak convergence}

In this section, we let $u_{k} \geq 0$ be a sequence of solutions to equation (1.2) verifying that

$$
J_{k}\left(u_{k}\right) \rightarrow \beta \text { as } k \rightarrow \infty \text { for some } \beta \in \mathbb{R},
$$

where $J_{k}$ is defined in (1.4). Testing equation (1.2) by $u_{k}$, we have

$$
\int_{\Sigma}\left(\left|\nabla_{g} u_{k}\right|^{2}+\tau_{k} u_{k}^{2}\right) d v_{g}=\int_{\Sigma} u_{k} f_{k}\left(x, u_{k}\right) d v_{g}
$$

It follows from 2.1 that

$$
\int_{\Sigma}\left(\left|\nabla_{g} u_{k}\right|^{2}+\tau_{k} u_{k}^{2}\right) d v_{g}=2 \beta+2 \int_{\Sigma} F_{k}\left(x, u_{k}\right) d v_{g}+o(1) .
$$

Hence

$$
\int_{\Sigma} u_{k} f_{k}\left(x, u_{k}\right) d v_{g}=2 \beta+2 \int_{\Sigma} F_{k}\left(x, u_{k}\right) d v_{g}+o(1)
$$


If $f_{k}$ satisfies the hypotheses (H1)-(H4), then we have

$$
\int_{\Sigma} u_{k} f_{k}\left(x, u_{k}\right) d v_{g} \leq C
$$

for some constant $C$. In view of (1.3), it follows from (2.2) and (2.3) that $u_{k}$ is bounded in $W^{1,2}(\Sigma, \mathbb{R})$. Hence there exists some $u_{\infty} \in W^{1,2}(\Sigma, \mathbb{R})$ such that up to a subsequence, $u_{k} \rightarrow u_{\infty}$ weakly in $W^{1,2}(\Sigma, \mathbb{R}), u_{k} \rightarrow u_{\infty}$ strongly in $L^{2}(\Sigma, \mathbb{R})$, and $u_{k} \rightarrow u_{\infty}$ a.e. in $\Sigma$. Similarly to [6], we then get that

$$
\lim _{k \rightarrow \infty} \int_{\Sigma} F_{k}\left(x, u_{k}\right) d v_{g}=\int_{\Sigma} F_{\infty}\left(x, u_{\infty}\right) d v_{g}
$$

that $u_{\infty}$ is a weak solution of $(1.5)$, and that $u_{\infty} \in C^{1}(\Sigma, \mathbb{R})$. In conclusion we obtained an analogue of ([6], Lemma1), namely

Lemma 2.1 Let $f_{k}$ be a sequence of functions satisfying (H1)-(H4). Let $u_{k} \geq 0$ be a sequence of solutions to (1.2), where $\tau_{k}$ is as defined in (1.3). If (2.1) holds, then $u_{k}$ is bounded in $W^{1,2}(\Sigma, \mathbb{R})$, and thus, up to a subsequence, $u_{k} \rightarrow u_{\infty}$ weakly in $W^{1,2}(\Sigma, \mathbb{R})$, where $u_{\infty} \in C^{1}(\Sigma, \mathbb{R})$ is a solution to (1.5). Also, there holds

$$
\lim _{k \rightarrow \infty} \int_{\Sigma}\left(\left|\nabla_{g} u_{k}\right|^{2}+\tau_{k} u_{k}^{2}\right) d v_{g}=2 \beta+2 \int_{\Sigma} F_{\infty}\left(x, u_{\infty}\right) d v_{g} .
$$

\section{Multibubble analysis}

In this section we shall use point wise estimate to find blow-up points of a sequence of solutions to the equation [1.2). This technique was first used by Druet [6] to deal with blow-up analysis for solutions to the equation (1.6). Assume $u_{k} \geq 0$ is a sequence of solutions to the equation (1.2) and (2.1) holds. From (2.2) and (2.3) we can find some constant $C$ such that

$$
\int_{\Sigma}\left(\left|\nabla_{g} u_{k}\right|^{2}+\tau_{k} u_{k}^{2}\right) d v_{g} \leq C .
$$

Then the Sobolev embedding theorem implies that for any $p>1$ there is some constant $C$ such that

$$
\int_{\Sigma} u_{k}^{p} d v_{g} \leq C
$$

These two properties are very important during the process of exhausting blow-up points. Precisely we have the following proposition which is analogous to ([6], Proposition 1), ([8], Theorem 4.2), ([9], Theorem 1 in the case $m=1)$ and ([3], Proposition 3.1).

Proposition 3.1 Let $(\Sigma, g)$ be a compact Riemannian surface without boundary, $\left(f_{k}\right)$ be a sequence of functions satisfying the hypotheses $(\mathrm{H} 1)-(\mathrm{H} 5)$, and $\left(u_{k}\right)$ be a sequence of smooth nonnegative solutions to (1.2) such that (2.1) holds. Assume that $\max _{\Sigma} u_{k} \rightarrow+\infty$ as $k \rightarrow \infty$. Then there exists $N \in \mathbb{N} \backslash\{0\}$, and up to a subsequence, there exist $N$ sequences of points $x_{i, k} \rightarrow x_{i}^{*} \in \Sigma$ and of positive real numbers $r_{i, k} \rightarrow 0$ as $k \rightarrow \infty$, where $r_{i, k}$ is defined by

$$
r_{i, k}^{-2}=u_{k}\left(x_{i, k}\right) f_{k}\left(x_{i, k}, u_{k}\left(x_{i, k}\right)\right),
$$


such that the following hold:

(i) For any $i=1,2, \cdots, N$, take an isothermal coordinate system $\left(U_{i}, \phi_{i} ;\left\{x^{1}, x^{2}\right\}\right)$ near $x_{i}^{*}$, where $U_{i} \subset \Sigma$ is a neighborhood of $x_{i}^{*}, \phi_{i}: U_{i} \rightarrow \Omega_{i} \subset \mathbb{R}^{2}$ is a diffeomorphism and $\phi_{i}\left(x_{i}^{*}\right)=(0,0)$. If we define

$$
\eta_{i, k}(x)=u_{k}\left(x_{i, k}\right)\left(\widetilde{u}_{k}\left(\widetilde{x}_{i, k}+r_{i, k} x\right)-u_{k}\left(x_{i, k}\right)\right)
$$

for all $x \in \Omega_{i, k}=\left\{x \in \mathbb{R}^{2}: \widetilde{x}_{i, k}+r_{i, k} \in \Omega_{i}\right\}$, where $\widetilde{x}_{i, k}=\phi_{i}\left(x_{i, k}\right)$ and $\widetilde{u}_{k}=u_{k} \circ \phi_{i}^{-1}$, then there holds

$$
\eta_{i, k}(x) \rightarrow \eta_{\infty}(x)=\log \frac{1}{1+|x|^{2} / 4} \quad \text { in } \quad C_{\mathrm{loc}}^{1}\left(\mathbb{R}^{2}\right) ;
$$

(ii) For any $1 \leq i \neq j \leq N$, there holds

$$
\frac{d_{g}\left(x_{i, k}, x_{j, k}\right)}{r_{i, k}} \rightarrow+\infty, \quad \text { as } \quad k \rightarrow \infty,
$$

where $d_{g}(\cdot, \cdot)$ denotes the geodesic distance between two points of $\Sigma$;

(iii) Define $R_{N, k}(x)=\min _{1 \leq i \leq N} d_{g}\left(x, x_{i, k}\right)$ for $x \in \Sigma$, then there exists a constant $C>0$ such that

$$
R_{N, k}^{2}(x) u_{k}(x) f_{k}\left(x, u_{k}(x)\right) \leq C
$$

uniformly in $x \in \Sigma$ and $k \in \mathbb{N}$.

Moreover, given any sequence of points $\left(x_{N+1, k}\right)$, it is impossible to extract a new subsequence from the previous one such that $(i)$ - (iii) hold with the sequences $\left(x_{i, k}\right), i=1, \cdots, N+1$.

Finally, we have $u_{k} \rightarrow u_{\infty}$ in $C_{\mathrm{loc}}^{1}(\Sigma \backslash \mathcal{S})$ as $k \rightarrow \infty$, where $\mathcal{S}=\left\{x_{1}^{*}, \cdots, x_{N}^{*}\right\}$, and $u_{\infty}$ is given in Lemma 2.1.

Proof. Similarly to [6, 8, 9, 3], we prove the proposition by several steps as follows.

Step 1. The first bubble.

Assume $u_{k}\left(x_{k}\right)=\max _{\Sigma} u_{k}$. If $u_{k}\left(x_{k}\right)$ is bounded, applying elliptic estimates to equation (1.2), we then have $u_{k} \rightarrow u_{\infty}$ in $C^{1}(\Sigma, \mathbb{R})$, where $u_{\infty}$ is given by Lemma 2.1 . Hereafter we assume $u_{k}\left(x_{k}\right) \rightarrow+\infty$. Set

$$
r_{k}^{-2}=u_{k}\left(x_{k}\right) f_{k}\left(x_{k}, u_{k}\left(x_{k}\right)\right) .
$$

It is clear that $r_{k} \rightarrow 0$ as $k \rightarrow \infty$.

Assume $x_{k} \rightarrow x^{*}$ as $k \rightarrow \infty$. Take an isothermal coordinate system $\left(U, \phi ;\left\{x^{1}, x^{2}\right\}\right)$ near $x^{*}$, where $U \subset \Sigma$ is a neighborhood of $x^{*}, \phi: U \rightarrow \Omega \subset \mathbb{R}^{2}$ is a diffeomorphism and $\phi\left(x^{*}\right)=(0,0)$. In such a coordinate system, the metric $g$ can be represented by

$$
g=e^{\psi}\left(d x^{1^{2}}+d x^{2^{2}}\right)
$$

for some smooth function $\psi: \Omega \rightarrow \mathbb{R}$ with $\psi(0,0)=0$. It follows that

$$
\nabla_{g}=e^{-\psi} \nabla_{\mathbb{R}^{2}}, \quad \Delta_{g}=-e^{-\psi} \Delta_{\mathbb{R}^{2}},
$$


where $\nabla_{\mathbb{R}^{2}}$ and $\Delta_{\mathbb{R}^{2}}$ denote the usual gradient operator and the Laplace operator of $\mathbb{R}^{2}$ respectively. The existence of isothermal coordinate system on Riemannian surface is a well-known fact in Riemannian geometry, see for example [15]. Define

$$
v_{k}(x)=\frac{\widetilde{u}_{k}\left(\widetilde{x}_{k}+r_{k} x\right)}{u_{k}\left(x_{k}\right)}
$$

for $x \in \Omega_{k}=\left\{x \in \mathbb{R}^{2}: \widetilde{x}_{k}+r_{k} x \in \Omega\right\}$, where $\widetilde{u}_{k}=u_{k} \circ \phi^{-1}, \widetilde{x}_{k}=\phi\left(x_{k}\right)$. It follows from (1.2), (3.5) and (3.6) that $v_{k}$ satisfies the following equation

$$
-\Delta_{\mathbb{R}^{2}} v_{k}(x)=e^{\psi\left(\widetilde{x_{k}}+r_{k} x\right)} \frac{\widetilde{f}_{k}\left(\widetilde{x}_{k}+r_{k} x, \widetilde{u}_{k}\left(\widetilde{x}_{k}+r_{k} x\right)\right)}{u_{k}^{2}\left(x_{k}\right) f_{k}\left(x_{k}, u_{k}\left(x_{k}\right)\right)}-e^{\psi\left(\widetilde{x}_{k}+r_{k} x\right)} r_{k}^{2} \widetilde{\tau}_{k}\left(\widetilde{x}_{k}+r_{k} x\right) v_{k}(x)
$$

on $\Omega_{k}$, where $\widetilde{f}_{k}\left(\widetilde{x}_{k}+r_{k} x, t\right)=f_{k}\left(\phi^{-1}\left(\widetilde{x}_{k}+r_{k} x\right), t\right)$. Note that $u_{k}\left(x_{k}\right)=\max _{\Sigma} u_{k}$ and $\Omega_{k} \rightarrow \mathbb{R}^{2}$ as $k \rightarrow \infty$. It follows from (3.7) that $v_{k}$ is uniformly bounded in $\mathbb{B}_{R}(0)$ for any fixed $R>0$. Since $\psi$ is smooth, $\psi(0,0)=0, \widetilde{x}_{k} \rightarrow(0,0)$ and $r_{k} \rightarrow 0$ as $k \rightarrow \infty, e^{\psi\left(\widetilde{x}_{k}+r_{k} x\right)}$ is also uniformly bounded in $\mathbb{B}_{R}(0)$ for any fixed $R>0$. Furthermore $e^{\psi\left(\widetilde{x}_{k}+r_{k} x\right)} \rightarrow 1$ locally uniformly in $\mathbb{R}^{2}$ as $k \rightarrow \infty$. By (H4) and (H5), we have for all $x \in \Omega_{k}$ and all $k$

$$
\frac{\widetilde{f}_{k}\left(\widetilde{x}_{k}+r_{k} x, \widetilde{u}_{k}\left(\widetilde{x}_{k}+r_{k} x\right)\right)}{f_{k}\left(x_{k}, u_{k}\left(x_{k}\right)\right)} \leq C .
$$

All these estimates together with (1.3) lead to

$$
\left\|-\Delta_{\mathbb{R}^{2}} v_{k}\right\|_{L^{\infty}\left(\mathbb{B}_{R}(0)\right)} \rightarrow 0 \text { as } k \rightarrow \infty, \forall R>0 .
$$

Applying elliptic estimates to (3.8), one gets $v_{k} \rightarrow v_{\infty}$ in $C_{\mathrm{loc}}^{1}\left(\mathbb{R}^{2}\right)$, where $v_{\infty}$ satisfies

$$
\left\{\begin{array}{l}
-\Delta_{\mathbb{R}^{2}} v_{\infty}=0 \text { in } \mathbb{R}^{2} \\
v_{\infty}(0)=1=\max _{\mathbb{R}^{2}} v_{\infty} .
\end{array}\right.
$$

The Liouville theorem for harmonic functions then leads to $v_{\infty} \equiv 1$. Therefore

$$
v_{k} \rightarrow 1 \text { in } C_{\mathrm{loc}}^{1}\left(\mathbb{R}^{2}\right) .
$$

Now we set

$$
\eta_{k}(x)=u_{k}\left(x_{k}\right)\left(\widetilde{u}_{k}\left(\widetilde{x}_{k}+r_{k} x\right)-u_{k}\left(x_{k}\right)\right)
$$

In view of (1.2), $\eta_{k}$ satisfies

$$
\begin{aligned}
-\Delta_{\mathbb{R}^{2}} \eta_{k}(x)= & e^{\psi\left(\widetilde{x_{k}}+r_{k} x\right)} \frac{\widetilde{f}_{k}\left(\widetilde{x}_{k}+r_{k} x, \widetilde{u}_{k}\left(\widetilde{x}_{k}+r_{k} x\right)\right)}{f_{k}\left(x_{k}, u_{k}\left(x_{k}\right)\right)} \\
& -e^{\psi\left(\widetilde{x}_{k}+r_{k} x\right)} \widetilde{\tau}_{k}\left(\widetilde{x}_{k}+r_{k} x\right) r_{k}^{2} u_{k}^{2}\left(x_{k}\right) v_{k}(x), \quad x \in \Omega_{k} .
\end{aligned}
$$

We claim that

$$
r_{k} u_{k}^{p}\left(x_{k}\right) \rightarrow 0 \quad \text { as } \quad k \rightarrow \infty, \quad \forall p>1 .
$$

Actually, it is clear that there exists some constant $c>0$ depending only on the diffeomorphism $\phi$ such that for any fixed $R>0$ and all large $k$

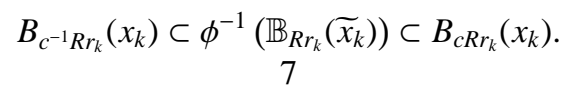


Here and throughout this paper we denote the geodesic ball centered at $x \in \Sigma$ with radius $r$ by $B_{r}(x)$, while the Euclidean ball centered at $x \in \mathbb{R}^{2}$ with radius $r$ by $\mathbb{B}_{r}(x)$. This together with (3.10), the mean value theorem for integral and the Hölder inequality leads to

$$
\begin{aligned}
r_{k} u_{k}^{p}\left(x_{k}\right) & =\frac{r_{k}}{\pi} \int_{\mathbb{B}_{1}(0)} u_{k}^{p}\left(x_{k}\right) d x \\
& =(1+o(1)) \frac{r_{k}}{\pi} \int_{\mathbb{B}_{1}(0)} \widetilde{u}_{k}^{p}\left(\widetilde{x}_{k}+r_{k} x\right) d x \\
& \leq(1+o(1)) \frac{r_{k}}{\pi^{1 / 3}}\left(\int_{\mathbb{B}_{1}(0)} \widetilde{u}_{k}^{3 p}\left(\widetilde{x}_{k}+r_{k} x\right) d x\right)^{1 / 3} \\
& \leq(1+o(1)) \frac{r_{k}^{1 / 3}}{\pi^{1 / 3}}\left(\int_{B_{c_{k}}\left(x_{k}\right)} u_{k}^{3 p} d v_{g}\right)^{1 / 3},
\end{aligned}
$$

where $o(1) \rightarrow 0$ as $k \rightarrow \infty$ for any fixed $p>1$. In view of (3.2), our claim (3.12) follows from (3.14) immediately.

For any fixed $R>0$ we let $\eta_{k}^{(1)}$ be a solution to the equation

$$
\left\{\begin{array}{l}
-\Delta_{\mathbb{R}^{2}} \eta_{k}^{(1)}=-\Delta_{\mathbb{R}^{2}} \eta_{k} \text { in } \mathbb{B}_{R}(0) \\
\eta_{k}^{(1)}=0 \text { on } \partial \mathbb{B}_{R}(0) .
\end{array}\right.
$$

In view of (3.11), we have by (3.9) and (3.12) that $\Delta_{\mathbb{R}^{2}} \eta_{k}$ is bounded in $L_{\text {loc }}^{\infty}\left(\mathbb{R}^{2}\right)$. Applying elliptic estimates to (3.15), we have

$$
\eta_{k}^{(1)} \rightarrow \eta_{\infty}^{(1)} \quad \text { in } \quad C^{1}\left(\mathbb{B}_{R}(0)\right) .
$$

Let $\eta_{k}^{(2)}=\eta_{k}-\eta_{k}^{(1)}$. Then $\eta_{k}^{(2)}$ satisfies

$$
-\Delta_{\mathbb{R}^{2}} \eta_{k}^{(2)}=0 \quad \text { in } \quad \mathbb{B}_{R}(0) .
$$

It follows from (3.16) and $\eta_{k} \leq 0$ that there exists some constant $C$ such that $\eta_{k}^{(2)}(x) \leq C$ for all $k$ and all $x \in \mathbb{B}_{R}(0)$. Applying the Harnack inequality to (3.17), we conclude that $\eta_{k}^{(2)}$ is uniformly bounded on $\mathbb{B}_{R / 2}(0)$. Hence $\eta_{k}$ is also uniformly bounded in $\mathbb{B}_{R / 2}(0)$. Applying elliptic estimates to (3.11), we obtain

$$
\eta_{k} \rightarrow \eta_{\infty} \quad \text { in } \quad C^{1}\left(\mathbb{B}_{R / 4}(0)\right) .
$$

This together with (H4), (H5) and (3.10) gives

$$
\frac{\widetilde{f}_{k}\left(\widetilde{x}_{k}+r_{k} x, \widetilde{u}_{k}\left(\widetilde{x}_{k}+r_{k} x\right)\right)}{f_{k}\left(x_{k}, u_{k}\left(x_{k}\right)\right)}=(1+o(1)) e^{(2+o(1)) \eta_{\infty}}
$$

for all $x \in \mathbb{B}_{R / 4}(0)$, where $o(1) \rightarrow 0$ as $k \rightarrow \infty$ uniformly in $x \in \mathbb{B}_{R / 4}(0)$. Inserting (3.12) and (3.18) into (3.11) and noting that $R>0$ is arbitrary we obtain

$$
\left\{\begin{array}{l}
-\Delta_{\mathbb{R}^{2}} \eta_{\infty}=e^{2 \eta_{\infty}} \text { in } \mathbb{R}^{2} \\
\eta_{\infty}(0)=0=\max _{\mathbb{R}^{2}} \eta_{\infty} .
\end{array}\right.
$$


Moreover, using (2.3), (3.5), (3.10), (3.13) and (3.18), we estimate for any fixed $R>0$

$$
\begin{aligned}
\int_{\mathbb{B}_{R}} e^{2 \eta_{\infty}} d x & =\lim _{k \rightarrow \infty} \int_{\mathbb{B}_{R}(0)} \frac{\widetilde{u}_{k}\left(\widetilde{x}_{k}+r_{k} x\right) \widetilde{f}_{k}\left(\widetilde{x}_{k}+r_{k} x, \widetilde{u}_{k}\left(\widetilde{x}_{k}+r_{k} x\right)\right)}{u_{k}\left(x_{k}\right) f_{k}\left(x_{k}, u_{k}\left(x_{k}\right)\right)} d x \\
& =\lim _{k \rightarrow \infty} \int_{\mathbb{B}_{R_{r_{k}}}\left(\widetilde{\widetilde{k}}_{k}\right)} \widetilde{u}_{k}(x) \widetilde{f}_{k}\left(x, \widetilde{u}_{k}(x)\right) d x \\
& \leq \limsup _{k \rightarrow \infty} \int_{B_{c r_{k}\left(x_{k}\right)}} u_{k} f_{k}\left(x, u_{k}\right) d v_{g} \leq C .
\end{aligned}
$$

It follows that

$$
\int_{\mathbb{R}^{2}} e^{2 \eta_{\infty}(x)} d x<\infty
$$

A result of Chen-Li [5] implies that

$$
\eta_{\infty}(x)=-\log \left(1+|x|^{2} / 4\right), \quad x \in \mathbb{R}^{2} .
$$

It follows from (3.13) that

$$
\int_{\mathbb{B}_{c^{-1} R_{r_{k}}}\left(\widetilde{x}_{k}\right)} \widetilde{u}_{k} \widetilde{f_{k}}\left(x, \widetilde{u}_{k}\right) e^{\psi(x)} d x \leq \int_{B_{R_{k}}\left(x_{k}\right)} u_{k} f_{k}\left(x, u_{k}\right) d v_{g} \leq \int_{\mathbb{B}_{c r_{k}}\left(\widetilde{x}_{k}\right)} \widetilde{u}_{k} \widetilde{f_{k}}\left(x, \widetilde{u}_{k}\right) e^{\psi(x)} d x .
$$

In view of (3.10) and (3.18), we have

$$
\begin{aligned}
\lim _{R \rightarrow \infty} \lim _{k \rightarrow \infty} \int_{\mathbb{B}_{c R_{k}}\left(\widetilde{x}_{k}\right)} \widetilde{u}_{k} \widetilde{f}_{k}\left(x, \widetilde{u}_{k}\right) e^{\psi(x)} d x & =\lim _{R \rightarrow \infty} \lim _{k \rightarrow \infty} \int_{\left.\mathbb{B}_{c^{-1}} \widetilde{R}_{r_{k}} \widetilde{\widetilde{x}}_{k}\right)} \widetilde{u}_{k} \widetilde{f}_{k}\left(x, \widetilde{u}_{k}\right) e^{\psi(x)} d x \\
& =\lim _{R \rightarrow \infty} \int_{\mathbb{B}_{c^{-1} R_{R}}(0)} e^{2 \eta_{\infty}} d x=\int_{\mathbb{R}^{2}} e^{2 \eta_{\infty}} d x .
\end{aligned}
$$

Therefore we obtain by 3.20

$$
\lim _{R \rightarrow \infty} \lim _{k \rightarrow \infty} \int_{B_{R_{r_{k}}}\left(x_{k}\right)} u_{k} f_{k}\left(x, u_{k}\right) d v_{g}=\int_{\mathbb{R}^{2}} e^{2 \eta_{\infty}}(x) d x=4 \pi .
$$

Step 2. Multi-bubble analysis.

In this step, we shall prove that there exists some positive integer $\ell$ such that the properties $\left(\mathcal{B}_{\ell}\right)$ and $\left(\mathcal{G}_{\ell}\right)$ hold. Namely, there exist $\ell$ sequences of points $\left(x_{i, k}\right) \subset \Sigma$ such that $x_{i, k} \rightarrow x_{i}^{*}$ as $k \rightarrow \infty, 1 \leq i \leq \ell$, and the following are satisfied:

$\left(\mathcal{B}_{\ell}^{1}\right)$ For every $i: 1 \leq i \leq \ell$, letting $r_{i, k}>0$ be given by $[3.3),\left(U_{i}, \phi_{i} ;\left\{x^{1}, x^{2}\right\}\right)$ be an isothermal coordinate system near $x_{i}^{*}$, where $U_{i} \subset \Sigma$ is a neighborhood of $x_{i}^{*}, \phi_{i}: U_{i} \rightarrow \Omega_{i} \subset \mathbb{R}^{2}$ is a diffeomorphism with $\phi_{i}\left(x_{i}^{*}\right)=(0,0)$, and letting $\eta_{i, k}$ be given by (3.4), we have that $r_{i, k} \rightarrow 0$ as $k \rightarrow \infty$ and

$$
\eta_{i, k}(x) \rightarrow \eta_{\infty}(x)=-\log \left(1+|x|^{2} / 4\right) \quad \text { in } \quad C_{\mathrm{loc}}^{1}\left(\mathbb{R}^{2}\right) \quad \text { as } \quad k \rightarrow \infty ;
$$

$\left(\mathcal{B}_{\ell}^{2}\right)$ For all $1 \leq i \neq j \leq \ell$,

$$
\frac{d_{g}\left(x_{i, k}, x_{j, k}\right)}{r_{i, k}} \rightarrow \infty \quad \text { as } \quad k \rightarrow \infty ;
$$


$\left(\mathcal{B}_{\ell}^{3}\right)$ The following energy identity holds

$$
\lim _{R \rightarrow \infty} \lim _{k \rightarrow \infty} \int_{\cup_{i=1}^{\ell} B_{R_{i, k}}\left(x_{i, k}\right)} u_{k} f_{k}\left(x, u_{k}\right) d v_{g}=4 \pi \ell ;
$$

$\left(\mathcal{G}_{\ell}\right)$ There exists a constant $C>0$ such that

$$
R_{\ell, k}^{2}(x) u_{k}(x) f_{k}\left(x, u_{k}(x)\right) \leq C
$$

for all $x \in \Sigma$ and all $k \in \mathbb{N}$. Here

$$
R_{\ell, k}(x)=\min _{1 \leq i \leq \ell} d_{g}\left(x, x_{i, k}\right) .
$$

From Step 1 , we know that $\left(\mathcal{B}_{1}\right)$ holds. Suppose for some $\ell \geq 1,\left(\mathcal{B}_{\ell}\right)$ holds but $\left(\mathcal{G}_{\ell}\right)$ does not hold. Choose $x_{\ell+1, k} \in \Sigma$ satisfying

$$
\begin{aligned}
R_{\ell, k}^{2}\left(x_{\ell+1, k}\right) u_{k}\left(x_{\ell+1, k}\right) f_{k}\left(x_{\ell+1, k}, u_{k}\left(x_{\ell+1, k}\right)\right) & =\max _{x \in \Sigma} R_{\ell, k}^{2}(x) u_{k}(x) f_{k}\left(x, u_{k}(x)\right) \\
& \rightarrow \quad+\infty \quad \text { as } k \rightarrow \infty .
\end{aligned}
$$

Let $r_{\ell+1, k}>0$ be as defined in (3.3). It follows from (3.3), (3.22), and (3.23) that $r_{\ell+1, k} \rightarrow 0$ as $k \rightarrow \infty$ and

$$
\lim _{k \rightarrow \infty} \frac{d_{g}\left(x_{\ell+1, k}, x_{i, k}\right)}{r_{\ell+1, k}}=+\infty, \quad \forall 1 \leq i \leq \ell
$$

Also we claim that

$$
\lim _{k \rightarrow \infty} \frac{d_{g}\left(x_{\ell+1, k}, x_{i, k}\right)}{r_{i, k}}=+\infty, \quad \forall 1 \leq i \leq \ell .
$$

Suppose not. There exists some constant $C$ such that for some $1 \leq i \leq \ell$, there holds

$$
d_{g}\left(x_{\ell+1, k}, x_{i, k}\right) \leq C r_{i, k} \text { for all } k \text {. }
$$

Hence we have

$$
R_{\ell, k}^{2}\left(x_{\ell+1, k}\right) u_{k}\left(x_{\ell+1, k}\right) f_{k}\left(x_{\ell+1, k}, u_{k}\left(x_{\ell+1, k}\right)\right) \leq C r_{i, k}^{2} u_{k}\left(x_{\ell+1, k}\right) f_{k}\left(x_{\ell+1, k}, u_{k}\left(x_{\ell+1, k}\right)\right)
$$

By $\left(\mathcal{B}_{\ell}^{1}\right)$, we estimate

$$
\begin{aligned}
r_{i, k}^{2} u_{k}\left(x_{\ell+1, k}\right) f_{k}\left(x_{\ell+1, k}, u_{k}\left(x_{\ell+1, k}\right)\right) & =\frac{1+o(1)}{\pi} \int_{\mathbb{B}_{r_{i, k}}\left(\widetilde{x}_{i, k}\right)} \widetilde{u}_{k}(x) \widetilde{f}_{k}\left(x, \widetilde{u}_{k}(x)\right) e^{\psi_{i}(x)} d x \\
& \leq \frac{1+o(1)}{\pi} \int_{\Sigma} u_{k}(x) f_{k}\left(x, u_{k}(x)\right) d v_{g} .
\end{aligned}
$$

This together with (2.3) implies that $r_{i, k}^{2} u_{k}\left(x_{\ell+1, k}\right) f_{k}\left(x_{\ell+1, k}, u_{k}\left(x_{\ell+1, k}\right)\right)$ is a bounded sequence, and whence (3.26) implies that $R_{\ell, k}^{2}\left(x_{\ell+1, k}\right) u_{k}\left(x_{\ell+1, k}\right) f_{k}\left(x_{\ell+1, k}, u_{k}\left(x_{\ell+1, k}\right)\right)$ is bounded. This contradicts (3.23). Hence our claim (3.25) holds, and thus $\left(\mathcal{B}_{\ell+1}^{2}\right)$ holds.

Assume $x_{\ell+1, k} \rightarrow x_{\ell+1}^{*}$ as $k \rightarrow \infty$. Take an isothermal coordinate system $\left(U_{\ell+1}, \phi_{\ell+1} ;\left\{x^{1}, x^{2}\right\}\right)$ near $x_{\ell+1}^{*}$, where $U_{\ell+1}$ is a neighborhood of $x_{\ell+1}^{*}, \phi_{\ell+1}: U_{\ell+1} \rightarrow \Omega_{\ell+1} \subset \mathbb{R}^{2}$ is a diffeomorphism with $\phi_{\ell+1}\left(x_{\ell+1}^{*}\right)=(0,0)$. In this coordinate system, the metric $g$ can be represented by

$$
g=e^{\psi_{t+1}}\left(d x^{1^{2}}+d x^{2^{2}}\right)
$$


for some smooth function $\psi_{\ell+1}: \Omega_{\ell+1} \rightarrow \mathbb{R}$ with $\psi_{\ell+1}(0,0)=0$. Also we have $\nabla_{g}=e^{-\psi_{\ell+1}} \nabla_{\mathbb{R}^{2}}$ and $\Delta_{g}=-e^{-\psi_{\ell+1}} \Delta_{\mathbb{R}^{2}}$.

Define

$$
v_{\ell+1, k}(x)=\frac{\widetilde{u}_{k}\left(\widetilde{x}_{\ell+1, k}+r_{\ell+1, k} x\right)}{u_{k}\left(x_{\ell+1, k}\right)}
$$

for $x \in \Omega_{\ell+1, k}=\left\{x \in \mathbb{R}^{2}: \widetilde{x}_{\ell+1, k}+r_{\ell+1, k} x \in \Omega_{\ell+1}\right\}$, where $\widetilde{x}_{\ell+1, k}=\phi_{\ell+1}\left(x_{\ell+1, k}\right), \widetilde{u}_{k}=u_{k} \circ \phi_{\ell+1}^{-1}$. Now we prove that

$$
v_{\ell+1, k} \rightarrow 1 \quad \text { in } \quad C_{\mathrm{loc}}^{1}\left(\mathbb{R}^{2}\right) \quad \text { as } \quad k \rightarrow \infty .
$$

In view of (1.2), $v_{\ell+1, k}$ satisfies the equation

$$
\begin{aligned}
-\Delta_{\mathbb{R}^{2}} v_{\ell+1, k}(x)= & e^{\psi_{\ell+1}\left(\widetilde{x}_{\ell+1, k}+r_{\ell+1, k}\right)} \frac{\widetilde{f}_{k}\left(\widetilde{x}_{\ell+1, k}+r_{\ell+1, k} x, \widetilde{u}_{k}\left(\widetilde{x}_{\ell+1, k}+r_{\ell+1, k} x\right)\right)}{u_{k}^{2}\left(x_{\ell+1, k}\right) f_{k}\left(x_{\ell+1, k}, u_{k}\left(x_{\ell+1, k}\right)\right)} \\
& -e^{\psi_{\ell+1}\left(\widetilde{x}_{\ell+1, k}+r_{\ell+1, k} x\right)} r_{\ell+1, k}^{2} \widetilde{\tau}_{k}\left(\widetilde{x}_{\ell+1, k}+r_{\ell+1, k} x\right) v_{\ell+1, k}(x)
\end{aligned}
$$

on $\Omega_{\ell+1, k}$, where $\widetilde{f}_{k}(x, t)=f_{k}\left(\phi_{\ell+1}^{-1}(x), t\right)$. By $(\underline{3.23})$, we have

$$
\begin{aligned}
& \widetilde{R}_{\ell, k}^{2}\left(\widetilde{x}_{\ell+1, k}+r_{\ell+1, k} x\right) \widetilde{u}_{k}\left(\widetilde{x}_{\ell+1, k}+r_{\ell+1, k} x\right) \widetilde{f}_{k}\left(\widetilde{x}_{\ell+1, k}+r_{\ell+1, k} x, \widetilde{u}_{k}\left(\widetilde{x}_{\ell+1, k}+r_{\ell+1, k} x\right)\right) \\
& \leq R_{\ell, k}^{2}\left(x_{\ell+1, k}\right) u_{k}\left(x_{\ell+1, k}\right) f_{k}\left(x_{\ell+1, k}, u_{k}\left(x_{\ell+1, k}\right)\right),
\end{aligned}
$$

where $\widetilde{R}_{\ell, k}=R_{\ell, k} \circ \phi_{\ell+1}^{-1}$. Fix any $i, 1 \leq i \leq \ell$. If $x_{\ell+1}^{*} \neq x_{i}^{*}$, noting that $d_{g}\left(\phi_{\ell+1}^{-1} \widetilde{x}_{\ell+1, k}+\right.$ $\left.\left.r_{\ell+1, k} x\right), x_{i, k}\right) \rightarrow d_{g}\left(x_{\ell+1}^{*}, x_{i}^{*}\right)$ and $d_{g}\left(x_{\ell+1, k}, x_{i, k}\right) \rightarrow d_{g}\left(x_{\ell+1}^{*}, x_{i}^{*}\right)$ as $k \rightarrow \infty$, we then have

$$
d_{g}\left(\phi_{\ell+1}^{-1}\left(\widetilde{x}_{\ell+1, k}+r_{\ell+1, k} x\right), x_{i, k}\right)=(1+o(1)) d_{g}\left(x_{\ell+1, k}, x_{i, k}\right),
$$

where $o(1) \rightarrow 0$ as $k \rightarrow \infty$ uniformly in $x \in \mathbb{B}_{R}(0)$. If $x_{\ell+1}^{*}=x_{i}^{*}$, since the Riemannian distance and the Euclidean distance are equivalent in the same local coordinate system, we then have $\left|\phi_{\ell+1}\left(x_{\ell+1, k}\right)-\phi_{\ell+1}\left(x_{i, k}\right)\right|=(1+o(1)) d_{g}\left(x_{\ell+1, k}, x_{i, k}\right)$. Recalling (3.24), we obtain for all $x \in \mathbb{B}_{R}(0)$

$$
\begin{aligned}
d_{g}\left(\phi_{\ell+1}^{-1}\left(\widetilde{x}_{\ell+1, k}+r_{\ell+1, k} x\right), x_{i, k}\right) & =(1+o(1))\left|\widetilde{x}_{\ell+1, k}+r_{\ell+1, k} x-\phi_{\ell+1}\left(x_{i, k}\right)\right| \\
& =(1+o(1)) d_{g}\left(x_{\ell+1, k}, x_{i, k}\right) .
\end{aligned}
$$

Hence we have (3.30) in any case. Combining (3.29) and (3.30), we obtain for $x \in \mathbb{B}_{R}(0)$

$$
\begin{aligned}
& v_{\ell+1, k}(x) \frac{\widetilde{f}_{k}\left(\widetilde{x}_{\ell+1, k}+r_{\ell+1, k} x, \widetilde{u}_{k}\left(\widetilde{x}_{\ell+1, k}+r_{\ell+1, k} x\right)\right)}{f_{k}\left(x_{\ell+1, k}, u_{k}\left(x_{\ell+1, k}\right)\right)} \\
& \leq \frac{\inf _{1 \leq i \leq \ell} d_{g}\left(x_{\ell+1, k}, x_{i, k}\right)^{2}}{\inf _{1 \leq i \leq \ell} d_{g}\left(\phi_{\ell+1}^{-1}\left(\widetilde{x}_{\ell+1, k}+r_{\ell+1, k} x\right), x_{i, k}\right)^{2}}=1+o(1),
\end{aligned}
$$

where $o(1) \rightarrow 0$ uniformly in $x \in \mathbb{B}_{R}(0)$. From (H4), we know that there exists $t_{0}>0$ such that

$$
\frac{f_{k}\left(x, t_{2}\right)}{f_{k}\left(x, t_{1}\right)} \geq e^{t_{2}^{2}-t_{1}^{2}} \text { for all } t_{1}, t_{2} \geq t_{0} \text {, and all } x \in \Sigma \text {. }
$$

If there exist some $R_{0}>0$ and a sequence of points $\left(z_{k}\right) \subset \mathbb{B}_{R_{0}}(0)$ such that $v_{\ell+1, k}\left(z_{k}\right) \rightarrow \alpha>1$ as $k \rightarrow \infty$, then we conclude by (3.32) and (H5) that

$$
v_{\ell+1, k}\left(z_{k}\right) \frac{\widetilde{f}_{k}\left(\widetilde{x}_{\ell+1, k}+r_{\ell+1, k} z_{k}, \widetilde{u}_{k}\left(\widetilde{x}_{\ell+1, k}+r_{\ell+1, k} z_{k}\right)\right)}{f_{k}\left(x_{\ell+1, k}, u_{k}\left(x_{\ell+1, k}\right)\right)} \geq \frac{\alpha+1}{11}>1
$$


for sufficiently large $k$, which contradicts (3.31). Therefore we obtain

$$
\limsup _{k \rightarrow \infty}\left\|v_{\ell+1, k}\right\|_{L^{\infty}\left(\mathbb{B}_{R}(0)\right)} \leq 1, \quad \forall R>0 .
$$

When $v_{\ell+1, k}(x)>1$, we have by (3.28) and (3.31), $\Delta_{\mathbb{R}^{2}} v_{\ell+1, k}(x)=o(1)$, where $o(1)$ is the same meaning as that of 3.31). When $v_{\ell+1, k}(x) \leq 1$, using (H4) and (H5), we also have $\Delta v_{\ell+1, k}(x)=$ $o(1)$, where $o(1) \rightarrow 0$ as $k \rightarrow \infty$ uniformly in all $x$ satisfying $v_{\ell+1, k}(x) \leq 1$ for sufficiently large $k$. Now applying elliptic estimates to equation (3.28), we obtain

$$
v_{\ell+1, k} \rightarrow v_{\ell+1, \infty} \quad \text { in } \quad C_{\mathrm{loc}}^{1}\left(\mathbb{R}^{2}\right)
$$

where $v_{\ell+1, \infty}$ is a solution to

$$
\left\{\begin{array}{l}
-\Delta_{\mathbb{R}^{2}} v_{\ell+1, \infty}=0 \quad \text { in } \quad \mathbb{R}^{2} \\
0 \leq v_{\ell+1, \infty} \leq 1 .
\end{array}\right.
$$

Note that $v_{\ell+1, \infty}(0)=1$. The Liouville theorem for harmonic functions leads to $v_{\ell+1, \infty} \equiv 1$. Whence (3.27) holds.

Define another sequence of blow-up functions by

$$
\eta_{\ell+1, k}(x)=u_{k}\left(x_{\ell+1, k}\right)\left(\widetilde{u}_{k}\left(\widetilde{x}_{\ell+1, k}+r_{\ell+1, k} x\right)-u_{k}\left(x_{\ell+1, k}\right)\right), \quad x \in \Omega_{\ell+1, k} .
$$

In the following, we will prove that $\left(\mathcal{B}_{\ell+1}^{1}\right)$ and $\left(\mathcal{B}_{\ell+1}^{3}\right)$ hold. By $(1.2), \eta_{\ell+1, k}$ satisfies the equation

$$
\begin{aligned}
-\Delta_{\mathbb{R}^{2}} \eta_{\ell+1, k}(x)= & e^{\psi_{\ell+1}\left(\widetilde{x}_{\ell+1, k}+r_{\ell+1, k}\right)} \frac{\widetilde{f}_{k}\left(\widetilde{x}_{\ell+1, k}+r_{\ell+1, k} x, \widetilde{u}_{k}\left(\widetilde{x}_{\ell+1, k}+r_{\ell+1, k} x\right)\right)}{f_{k}\left(x_{\ell+1, k}, u_{k}\left(x_{\ell+1, k}\right)\right)} \\
& -e^{\psi_{\ell+1}\left(\widetilde{x}_{\ell+1, k}+r_{\ell+1, k} x\right)} \widetilde{\tau}_{k}\left(\widetilde{x}_{\ell+1, k}+r_{\ell+1, k} x\right) r_{\ell+1, k}^{2} u_{k}^{2}\left(x_{\ell+1, k}\right) v_{\ell+1, k}(x)
\end{aligned}
$$

on $\Omega_{\ell+1, k}$. We claim that for any fixed $R>0$,

$$
\limsup _{k \rightarrow \infty} \eta_{\ell+1, k}(x) \leq 0 \text { uniformly in } x \in \mathbb{B}_{R}(0) .
$$

For otherwise, we may take a sequence of points $\left(y_{k}\right) \subset \mathbb{B}_{R}(0)$ such that $\eta_{\ell+1, k}\left(y_{k}\right) \geq \beta>0$ for all sufficiently large $k$. By (H4), (H5) and (3.27), we obtain

$$
\begin{aligned}
\frac{\widetilde{f}_{k}\left(\widetilde{x}_{\ell+1, k}+r_{\ell+1, k} y_{k}, \widetilde{u}_{k}\left(\widetilde{x}_{\ell+1, k}+r_{\ell+1, k} y_{k}\right)\right)}{f_{k}\left(x_{\ell+1, k}, u_{k}\left(x_{\ell+1, k}\right)\right)} & =(1+o(1)) e^{\left.\widetilde{u}_{k}^{2} \widetilde{x_{\ell+1, k}}+r_{\ell+1, k y_{k}}\right)-u_{k}^{2}\left(x_{\ell+1, k}\right)} \\
& =(1+o(1)) e^{(2+o(1)) \eta_{\ell+1, k}\left(y_{k}\right)} \\
& \geq 1+2 \beta+o(1) .
\end{aligned}
$$

This together with (3.31) leads to

$$
1+2 \beta+o(1) \leq 1+o(1)
$$

which is impossible when $k$ is sufficiently large. Hence our claim (3.35) holds. By (3.27), using the same method of deriving (3.12), we conclude

$$
r_{\ell+1, k}^{2} u_{k}^{2}\left(x_{\ell+1, k}\right) \rightarrow 0 \quad \text { as } \quad k \rightarrow \infty .
$$


Combining (3.27) and (3.33)-(3.36), similarly as we did in Step 1, we arrive at

$$
\eta_{\ell+1, k}(x) \rightarrow \eta_{\infty}(x) \text { in } C_{\mathrm{loc}}^{1}\left(\mathbb{R}^{2}\right) \text { as } k \rightarrow \infty,
$$

where $\eta_{\infty}(x)=-\log \left(1+|x|^{2} / 4\right)$ is the unique solution to 3.19$)$. Hence $\left(\mathcal{B}_{\ell+1}^{1}\right)$ holds.

Moreover, using the same method for proving (3.21), we arrive at

$$
\lim _{R \rightarrow \infty} \lim _{k \rightarrow \infty} \int_{B_{R_{\ell+1, k}}\left(x_{\ell+1, k}\right)} u_{k} f_{k}\left(x, u_{k}\right) d v_{g}=\int_{\mathbb{R}^{2}} e^{2 \eta_{\infty}(x)} d x=4 \pi .
$$

Thus $\left(\mathcal{B}_{\ell+1}^{3}\right)$ holds.

Actually, we have proved that if $\left(\mathcal{B}_{\ell}\right)$ holds but $\left(\mathcal{G}_{\ell}\right)$ does not hold, then $\left(\mathcal{B}_{\ell+1}\right)$ holds. Note that

$$
\int_{\Sigma} u_{k} f_{k}\left(x, u_{k}\right) d v_{g} \geq \sum_{i=1}^{\ell+1} \int_{B_{R_{r, k}}\left(x_{i, k}\right)} u_{k} f_{k}\left(x, u_{k}\right) d v_{g}=4(\ell+1) \pi
$$

In view of $(2.3)$, the process must be terminate after finite steps. This ends the proof of Step 2.

\section{Step 3. Exhaustion of blow-up points.}

It follows from Step 2 that there exists some $\ell \in \mathbb{N} \backslash\{0\}$ and $\ell$ sequences of points $\left(x_{i, k}\right)$, $i=1, \cdots, \ell$, such that $\left(\mathcal{B}_{\ell}\right)$ and $\left(\mathcal{G}_{\ell}\right)$ hold. If there exists a sequence of points $\left(x_{\ell+1, k}\right)$ of $\Sigma$ such that after extracting a new subsequence from the previous one, $\left(\mathcal{B}_{\ell+1}\right)$ and $\left(\mathcal{G}_{\ell+1}\right)$ hold, we add this sequence of points, and so on. The process necessarily terminates because of (2.3) and (3.37). Therefore there exists some $N \in \mathbb{N} \backslash\{0\}$ and $N$ sequences of points $\left(x_{i, k}\right), i=1, \cdots, N$, such that $\left(\mathcal{B}_{N}\right)$ and $\left(\mathcal{G}_{N}\right)$ hold and such that, given any sequence of points $\left(x_{N+1, k}\right)$, it is impossible to extract a new subsequence from the previous one such that $\left(\mathcal{B}_{N+1}\right)$ and $\left(\mathcal{G}_{N+1}\right)$ hold with sequences $\left(x_{i, k}\right), i=1, \cdots, N+1$.

Step 4. Convergence away from blow-up points.

Set $\mathcal{S}=\left\{x_{1}^{*}, \cdots, x_{N}^{*}\right\}$. We will prove that $u_{k} \rightarrow u_{\infty}$ in $C_{\text {loc }}^{1}(\Sigma \backslash \mathcal{S})$. In view of $\left(\mathcal{G}_{N}\right)$, given any compact set $K \subset \Sigma \backslash \mathcal{S}$, there exists a constant $C$ such that

$$
u_{k}(x) f_{k}\left(x, u_{k}(x)\right) \leq C \text { for all } x \in K \text { and all } k .
$$

If $u_{k}(x)>1$ for some $x \in K$, then $f_{k}\left(x, u_{k}(x)\right) \leq C_{K}$. If $u_{k}(x) \leq 1$ for some $x \in K$, then (H2) implies that $f_{k}\left(x, u_{k}(x)\right)$ is bounded uniformly in $x$ with $u_{k}(x) \leq 1$. Thus, for all $x \in K, f_{k}\left(x, u_{k}(x)\right)$ is bounded in $L^{\infty}(K)$. In view of (1.3) and (3.2), applying elliptic estimates to the equation

$$
\Delta_{g} u_{k}(x)+\tau_{k}(x) u_{k}(x)=f_{k}\left(x, u_{k}(x)\right), \quad x \in K,
$$

we obtain the convergence $u_{k} \rightarrow u_{0}$ in $C_{\mathrm{loc}}^{1}(\Sigma \backslash \mathcal{S})$.

Combining the above four steps, we complete the proof of Proposition 3.1. 


\section{Gradient estimate}

Let $u_{k} \geq 0$ be a sequence of solutions to (1.2). In this section we shall establish a gradient estimate on $u_{k}$, which can be viewed as a version on manifolds of ([6], Proposition 2). Precisely we have the following result.

Proposition 4.1 Let $(\Sigma, g)$ be a compact Riemannian surface without boundary, $f_{k}$ be a sequence of functions satisfying (H1)-(H5), and $u_{k} \geq 0$ be a sequence of smooth solutions to equation (1.2) such that (2.1) holds. Assume that $\max _{\Sigma} u_{k} \rightarrow+\infty$ as $k \rightarrow \infty$. Let $N \in \mathbb{N} \backslash\{0\}$ and the sequences $x_{i, k}, i=1, \cdots, N$, be given by Proposition 3.1. Then there exists a uniform constant $C$ such that

$$
R_{N, k}(x) u_{k}(x)\left|\nabla_{g} u_{k}(x)\right| \leq C
$$

for all $x \in \Sigma$ and all $k$, where $R_{N, k}(x)$ is defined as in (3.22).

Proof. Choose $y_{k} \in \Sigma$ such that

$$
R_{N, k}\left(y_{k}\right) u_{k}\left(y_{k}\right)\left|\nabla_{g} u_{k}\left(y_{k}\right)\right|=\max _{x \in \Sigma} R_{N, k}(x) u_{k}(x)\left|\nabla_{g} u_{k}(x)\right| .
$$

Suppose by contradiction that

$$
R_{N, k}\left(y_{k}\right) u_{k}\left(y_{k}\right)\left|\nabla_{g} u_{k}\left(y_{k}\right)\right| \rightarrow+\infty \quad \text { as } \quad k \rightarrow \infty .
$$

Set

$$
s_{k}=R_{N, k}\left(y_{k}\right) .
$$

By Proposition 3.1, we have $u_{k} \rightarrow u_{\infty}$ in $C_{\mathrm{loc}}^{1}\left(\Sigma \backslash\left\{x_{1}^{*}, \cdots, x_{N}^{*}\right\}\right)$, which together with (4.2) implies that $s_{k} \rightarrow 0$ as $k \rightarrow \infty$. Without loss of generality, we may assume that $y_{k} \rightarrow x_{1}^{*}$ as $k \rightarrow \infty$, $x_{1}^{*}=\cdots=x_{\ell}^{*}$ for some $1 \leq \ell \leq N$, and $x_{j}^{*} \neq x_{1}^{*}$ for any $j \in\{\ell+1, \cdots, N\}$. Take an isothermal coordinate system $\left(U, \phi ;\left\{x^{1}, x^{2}\right\}\right)$ near $x_{1}^{*}$, where $U$ is a neighborhood of $x_{1}^{*} \in \Sigma, \phi: U \rightarrow \Omega \subset \mathbb{R}^{2}$ is a diffeomorphism with $\phi\left(x_{1}^{*}\right)=(0,0)$. In this coordinate system the metric $g$ can be represented by $g=e^{\psi}\left(d x^{1^{2}}+d x^{2}\right)$, where $\psi: \Omega \rightarrow \mathbb{R}$ is a smooth function with $\psi(0,0)=0$. Denote $\widetilde{y}_{k}=\phi\left(y_{k}\right), \widetilde{u}_{k}=u_{k} \circ \phi^{-1}$. We set

$$
v_{k}(y)=\widetilde{u}_{k}\left(\widetilde{y}_{k}+s_{k} y\right)
$$

for $y \in \Omega_{k}=\left\{y \in \mathbb{R}^{2}: \widetilde{y}_{k}+s_{k} y \in \Omega\right\}$. Define

$$
y_{i, k}=\frac{\widetilde{x}_{i, k}-\widetilde{y}_{k}}{s_{k}} \in \Omega_{k}, \quad i=1, \cdots, \ell,
$$

and

$$
\widetilde{S}_{k}=\left\{y_{1, k}, \cdots, y_{\ell, k}\right\} .
$$

Since $s_{k} \rightarrow 0$, we have $\Omega_{k} \rightarrow \mathbb{R}^{2}$ as $k \rightarrow \infty$. Denote

$$
\widetilde{S}=\lim _{k \rightarrow \infty} \widetilde{S}_{k}
$$

By (4.3) and the fact $\psi(0,0)=0$, we have

$$
\begin{aligned}
d_{\mathbb{R}^{2}}\left(0, \widetilde{S}_{k}\right) & =\inf _{1 \leq i \leq \ell}\left|y_{i, k}\right|=\inf _{1 \leq i \leq \ell} \frac{\left|\widetilde{x}_{i, k}-\widetilde{y}_{k}\right|}{s_{k}} \\
& =\inf _{1 \leq i \leq \ell} \frac{(1+o(1)) d_{g}\left(x_{i, k}, y_{k}\right)}{s_{k}} \\
& =1+o(1),
\end{aligned}
$$


and thus

$$
d_{\mathbb{R}^{2}}(0, \widetilde{S})=1,
$$

where $d_{\mathbb{R}^{2}}(\cdot, \cdot)$ denotes the Euclidean distance of $\mathbb{R}^{2}$. Clearly, $v_{k}(y)$ satisfies

$$
-\Delta_{\mathbb{R}^{2}} v_{k}(y)=e^{\psi\left(\widetilde{y}_{k}+s_{k} y\right)} s_{k}^{2}\left(\widetilde{f}_{k}\left(\widetilde{y}_{k}+s_{k} y, \widetilde{u}_{k}\left(\widetilde{y}_{k}+s_{k} y\right)\right)-\widetilde{\tau}_{k}\left(\widetilde{y}_{k}+s_{k} y\right) v_{k}(y)\right)
$$

for $y \in \Omega_{k}$. By (iii) of Proposition 3.1, we have

$$
\widetilde{R}_{N, k}\left(\widetilde{y}_{k}+s_{k} y\right)^{2} v_{k}(y) \widetilde{f_{k}}\left(\widetilde{y}_{k}+s_{k} y, v_{k}(y)\right) \leq C
$$

for some constant $C$ independent of $k$. Note that

$$
\begin{aligned}
\widetilde{R}_{N, k}\left(\widetilde{y}_{k}+s_{k} y\right) & =R_{N, k}\left(\phi^{-1}\left(\widetilde{y}_{k}+s_{k} y\right)\right) \\
& \left.=\inf _{1 \leq i \leq \ell} d_{g}\left(\phi^{-1} \widetilde{y}_{k}+s_{k} y\right), x_{i, k}\right) \\
& =(1+o(1)) \inf _{1 \leq i \leq \ell} d_{\mathbb{R}^{2}}\left(\widetilde{y}_{k}+s_{k} y, \widetilde{x}_{i, k}\right) \\
& =(1+o(1)) s_{k} d_{\mathbb{R}^{2}}\left(y, \widetilde{S}_{k}\right) .
\end{aligned}
$$

Combining (4.6) and (4.7), we have

$$
s_{k}^{2} v_{k}(y) \widetilde{f_{k}}\left(\widetilde{y}_{k}+s_{k} y, v_{k}(y)\right) \leq \frac{C}{d_{\mathbb{R}^{2}}\left(y, \widetilde{S}_{k}\right)^{2}},
$$

which together with (H1) and (H2) leads to

$$
0 \leq s_{k}^{2} \widetilde{f}_{k}\left(\widetilde{y}_{k}+s_{k} y, v_{k}(y)\right) \leq \frac{C}{d_{\mathbb{R}^{2}}\left(y, \widetilde{S}_{k}\right)^{2}} .
$$

In view of (3.2), we estimate for any $p>1$ and any $R>0$,

$$
\begin{aligned}
\int_{\mathbb{B}_{R}(0)}\left(s_{k}^{2} v_{k}(y)\right)^{p} d y & =s_{k}^{2 p} \int_{\mathbb{B}_{R}(0)} \widetilde{u}_{k}\left(\widetilde{y}_{k}+s_{k} y\right)^{p} d y \\
& \leq C s_{k}^{2 p-2} \int_{\Sigma} u_{k}^{p} d v_{g} \\
& \rightarrow 0 \text { as } \quad k \rightarrow \infty .
\end{aligned}
$$

Denote for any $R>0$

$$
A_{R}=\mathbb{B}_{R}(0) \backslash \cup_{y \in \mathcal{S}} \mathbb{B}_{1 / R}(y) .
$$

Clearly there exists some $R_{0}>0$ such that $A_{R / 4}$ is necessarily smooth bounded domain provided that $R \geq R_{0}$. Now we take $R \geq R_{0}$. In view of (1.3), (4.5), (4.9), and (4.10), we arrive at

$$
\lim _{k \rightarrow \infty}\left\|\Delta_{\mathbb{R}^{2}} v_{k}\right\|_{L^{p}\left(A_{R}\right)}=0, \quad \forall R \geq R_{0}, \quad \forall p>1 .
$$

Let $w_{k}$ satisfy

$$
\left\{\begin{array}{l}
-\Delta_{\mathbb{R}^{2}} w_{k}=-\Delta_{\mathbb{R}^{2}} v_{k} \text { in } A_{R} \\
w_{k}=0 \text { on } \partial A_{R} .
\end{array}\right.
$$


It follows from (4.10) and elliptic estimates that there exists some function $w$ such that

$$
w_{k} \rightarrow w \text { in } C^{1}\left(\overline{A_{R}}\right) .
$$

In particular, $w_{k}$ is uniformly bounded in $A_{R}$. While $v_{k}-w_{k}$ satisfies

$$
\left\{\begin{array}{l}
-\Delta_{\mathbb{R}^{2}}\left(v_{k}-w_{k}\right)=0 \quad \text { in } \quad A_{R} \\
v_{k}-w_{k}=v_{k} \quad \text { on } \quad \partial A_{R}
\end{array}\right.
$$

We claim that

$$
v_{k}(0) \rightarrow+\infty \quad \text { as } \quad k \rightarrow \infty .
$$

For otherwise, $\left(v_{k}(0)-w_{k}(0)\right)$ would be a bounded sequence. Noting that $v_{k}-w_{k}$ has a lower bound in $A_{R}$, applying Harnack's inequality to (4.11), we obtain

$$
\left\|v_{k}-w_{k}\right\|_{L^{\infty}\left(A_{R / 2}\right)} \leq C
$$

for some constant $C$ depending only on $R$, and whence $v_{k}$ is bounded in $C^{1}\left(A_{R / 4}\right)$. In view of (4.4), this leads to

$$
v_{k}(0)\left|\nabla_{\mathbb{R}^{2}} v_{k}(0)\right| \leq C .
$$

While (4.1) and (4.2) implies

$$
v_{k}(0)\left|\nabla_{\mathbb{R}^{2}} v_{k}(0)\right| \rightarrow+\infty \quad \text { as } \quad k \rightarrow \infty
$$

This is a contradiction. Hence our claim (4.12) follows.

Replacing $v_{k}$ by $v_{k} / v_{k}(0)$ in the above estimates, we obtain

$$
\frac{v_{k}}{v_{k}(0)} \rightarrow 1 \quad \text { in } \quad C_{\mathrm{loc}}^{1}\left(\mathbb{R}^{2} \backslash \mathcal{S}\right)
$$

as $k \rightarrow \infty$. For $y \in \Omega_{k}$, we set

$$
\widetilde{v}_{k}(y)=\frac{v_{k}(y)-v_{k}(0)}{\left|\nabla_{\mathbb{R}^{2}} v_{k}(0)\right|} .
$$

It follows from (4.1) and 4.7) that

$$
v_{k}(y)\left|\nabla_{\mathbb{R}^{2}} v_{k}(y)\right| \leq(1+o(1)) \frac{v_{k}(0) \mid \nabla_{\mathbb{R}^{2} v_{k}(0) \mid}}{d_{\mathbb{R}^{2}}\left(y, \widetilde{S}_{k}\right)}, \quad y \in \Omega_{k} \backslash \widetilde{S}_{k} .
$$

This together with 4.14 gives

$$
\left|\nabla_{\mathbb{R}^{2}} \widetilde{v}_{k}(y)\right| \leq \frac{1+o(1)}{d_{\mathbb{R}^{2}}(y, \mathcal{S})},
$$

where $o(1) \rightarrow 0$ as $k \rightarrow \infty$ locally uniformly in $y \in \mathbb{R}^{2} \backslash \mathcal{S}$. Since $\widetilde{v}_{k}(0)=0$, it follows from (4.15) that $\widetilde{v}_{k}$ is uniformly bounded in $C^{1}\left(A_{R}\right)$ for any $R>0$. In view of (4.5) and (4.14), we have

$$
\begin{aligned}
-\Delta_{\mathbb{R}^{2}} \widetilde{v}_{k}(y) & =-(1+o(1)) \frac{v_{k}(y) \Delta_{\mathbb{R}^{2}} v_{k}(y)}{v_{k}(0)\left|\nabla_{\mathbb{R}^{2}} v_{k}(0)\right|} \\
& =\frac{1+o(1)}{v_{k}(0)\left|\nabla_{\mathbb{R}^{2}} v_{k}(0)\right|} e^{\psi\left(\widetilde{y}_{k}+s_{k} y\right)} s_{k}^{2} v_{k}(y)\left\{\widetilde{f}_{k}\left(\widetilde{y}_{k}+s_{k} y, v_{k}(y)\right)-\widetilde{\tau}_{k}\left(\widetilde{y}_{k}+s_{k} y\right) v_{k}(y)\right\}
\end{aligned}
$$


for $y \in \Omega_{k}$. Similarly to (4.10), $s_{k}^{2} v_{k}^{2}$ is bounded in $L_{\text {loc }}^{p}\left(\mathbb{R}^{2}\right)$ for any $p>1$. In view of (4.8) and (4.13), applying elliptic estimates to the equation (4.16), we have

$$
\widetilde{v}_{k} \rightarrow \widetilde{v} \text { in } C_{\mathrm{loc}}^{1}\left(\mathbb{R}^{2} \backslash \mathcal{S}\right) \quad \text { as } \quad k \rightarrow \infty,
$$

where $\widetilde{v}$ satisfies

$$
\Delta_{\mathbb{R}^{2}} \widetilde{v}=0 \quad \text { in } \quad \mathbb{R}^{2} \backslash \mathcal{S}, \quad \widetilde{v}(0)=0, \quad\left|\nabla_{\mathbb{R}^{2}} \widetilde{v}(0)\right|=1,
$$

and

$$
\left|\nabla_{\mathbb{R}^{2}} \widetilde{v}(y)\right| \leq \frac{1}{d_{\mathbb{R}^{2}}(y, \mathcal{S})}, \quad y \in \mathbb{R}^{2} \backslash \mathcal{S} .
$$

Let $\hat{y} \in \mathcal{S}$. For any $0<r<d_{\mathbb{R}^{2}}(\hat{y}, \mathcal{S} \backslash\{\hat{y}\}) / 2$, since

$$
\begin{aligned}
\int_{\mathbb{B}_{r}(\hat{y})} v_{k} \Delta_{\mathbb{R}^{2}} v_{k} d y & =\int_{\mathbb{B}_{r}(\hat{y})} \widetilde{u}_{k}\left(\widetilde{y}_{k}+s_{k} y\right) s_{k}^{2} \Delta_{\mathbb{R}^{2}} \widetilde{u}_{k}\left(\widetilde{y}_{k}+s_{k} y\right) d y \\
& =\int_{\mathbb{B}_{s_{k} r}\left(\widetilde{\widetilde{y}}_{k}+s_{k} \hat{y}\right)} \widetilde{u}_{k}(x) \Delta_{\mathbb{R}^{2}} \widetilde{u}_{k}(x) d x \\
& =-\int_{\phi^{-1}\left(\mathbb{B}_{s_{k} r}\left(\widetilde{\gamma}_{k}+s_{k} \hat{y}\right)\right)} u_{k} \Delta_{g} u_{k} d v_{g},
\end{aligned}
$$

we get by (1.3), (2.3) and (3.2)

$$
\left|\int_{\mathbb{B}_{r}(\hat{y})} v_{k} \Delta_{\mathbb{R}^{2}} v_{k} d y\right| \leq \int_{\Sigma}\left(u_{k} f_{k}\left(x, u_{k}\right)+\tau_{k} u_{k}^{2}\right) d v_{g} \leq C .
$$

Similarly we have by (3.1)

$$
\int_{\mathbb{B}_{r}(\hat{y})}\left|\nabla_{\mathbb{R}^{2}} v_{k}\right|^{2} d y \leq \int_{\Sigma}\left|\nabla_{g} u_{k}\right|^{2} d v_{g} \leq C .
$$

It then follows that

$$
\int_{\partial \mathbb{B}_{r}(\hat{y})} v_{k} \partial_{v} v_{k} d \sigma=\int_{\mathbb{B}_{r}(\hat{y})}\left|\nabla_{\mathbb{R}^{2}} v_{k}\right|^{2} d y-\int_{\mathbb{B}_{r}(\hat{y})} v_{k} \Delta_{\mathbb{R}^{2}} v_{k} d y=O(1) .
$$

While (4.14) and (4.17) lead to

$$
\int_{\partial \mathbb{B}_{r}(\hat{y})} v_{k} \partial_{\nu} v_{k} d \sigma=v_{k}(0)\left|\nabla_{\mathbb{R}^{2}} v_{k}(0)\right|\left(\int_{\partial \mathbb{B}_{r}(\hat{y})} \partial_{\nu} \widetilde{v} d \sigma+o(1)\right) .
$$

This together with (4.13) gives for any $0<r<d_{\mathbb{R}^{2}}(\hat{y}, \mathcal{S} \backslash\{\hat{y}\}) / 2$

$$
\int_{\partial \mathbb{B}_{r}(\hat{y})} \partial_{\nu} \widetilde{v} d \sigma=0
$$

which leads to

$$
\frac{d}{d r}\left(\frac{1}{2 \pi r} \int_{\partial \mathbb{B}_{r}(\hat{y})} \widetilde{v} d \sigma\right)=\frac{1}{2 \pi r} \int_{\partial \mathbb{B}_{r}(\hat{y})} \partial_{\nu} \widetilde{v} d \sigma=0 .
$$

Hence there exists some constant $\alpha$ depending only on $\hat{y}$ such that

$$
\frac{1}{2 \pi r} \int_{\partial \mathbb{B}_{r}(\hat{y})} \tilde{v} d \sigma=\alpha, \quad \forall 0<r<d_{\mathbb{R}^{2}}(\hat{y}, \mathcal{S} \backslash\{\hat{y}\}) / 2 .
$$


Given any $y \in \partial \mathbb{B}_{r}(\hat{y})$. (4.20) permits us to take $y^{*} \in \partial \mathbb{B}_{r}(\hat{y})$ such that $\widetilde{v}\left(y^{*}\right)=\alpha$. It then follows from (4.19) that $|\widetilde{v}(y)-\alpha| \leq \pi$. This indicates that $\widetilde{v}$ is bounded near $\hat{y}$. Since this is true for all $\hat{y} \in \mathcal{S}$, we conclude that $\widetilde{v}$ is a smooth harmonic function in $\mathbb{R}^{2}$. By the mean value equality,

$$
\int_{\partial \mathbb{B}_{R}(0)} \widetilde{v} d \sigma=0, \quad \forall R>0
$$

This together with 4.19) implies that $\widetilde{v}$ is bounded in $L^{\infty}\left(\mathbb{R}^{2}\right)$. Actually we can take $z \in \partial \mathbb{B}_{R}(0)$ such that $\widetilde{v}_{k}(z)=0$, in view of 4.19), we then have for all $y \in \partial \mathbb{B}_{R}(0)$

$$
\left|\widetilde{v}_{k}(y)\right|=\widetilde{v}_{k}(y)-\widetilde{v}_{k}(z)\left|\leq \pi R \sup _{\partial \mathbb{B}_{R}(0)}\right| \nabla_{\mathbb{R}^{2}} \widetilde{v} \mid \leq 2 \pi,
$$

provided that $R>2 \sup _{\hat{y} \in \mathcal{S}}|\hat{y}|$. Note again that $\widetilde{v}(0)=0$. Applying the Liouville theorem to (4.18), we have $\widetilde{v} \equiv 0$, which contradicts the fact that $\left|\nabla_{\mathbb{R}^{2}} \widetilde{v}(0)\right|=1$. This completes the proof of the proposition.

\section{Quantization}

In this section we prove quantization results for equation (1.2). Let $x_{1}^{*}, \cdots, x_{N}^{*}$ be as in Proposition 3.1. For some $1 \leq i \leq N, x_{i}^{*}$ is called a simple blow-up point if $N=1$ or $x_{j} \neq x_{i}$ for all $j \in\{1, \cdots, N\} \backslash\{i\}$; Otherwise we call $x_{i}^{*}$ a non-simple blow-up point. In the following, we distinguish between these two types of points to proceed.

\subsection{Quantization for simple blow-up points}

Let $x_{i}^{*}$ be a simple blow-up point. Take an isothermal coordinate system $\left(U_{i}, \phi_{i} ;\left\{x^{1}, x^{2}\right\}\right)$ near $x_{i}^{*}$, where $U_{i} \subset \Sigma$ is a neighborhood of $x_{i}^{*}$ such that $x_{j}^{*} \notin \bar{U}_{i}$, the closure of $U_{i}$, for all $j \in\{1, \cdots, N\} \backslash\{i\}$. As before $\phi_{i}: U_{i} \rightarrow \Omega \subset \mathbb{R}^{2}$ is a diffeomorphism with $\phi_{i}\left(x_{i}^{*}\right)=(0,0)$. Particularly we can find some $\delta>0$ such that $\mathbb{B}_{2 \delta}(0) \subset \Omega$. In this coordinate system, the metric $g$ writes as $g=e^{\psi_{i}}\left(d x^{1^{2}}+d x_{2}{ }^{2}\right)$ for some smooth function $\psi_{i}: \Omega \rightarrow \mathbb{R}$ with $\psi_{i}(0,0)=0$. In this subsection we prove the following quantization result.

Proposition 5.1 Let $u_{k}, u_{\infty}, \tau_{k}, \tau_{\infty}, x_{i, k}$ and $x_{i}^{*}$ be as in Proposition 3.1. Suppose that $x_{i}^{*}$ is a simple blow-up point. Then up to a subsequence, there exists some positive integer $I^{(i)}$ such that

$$
\lim _{k \rightarrow \infty} \int_{U_{i}}\left(\left|\nabla_{g} u_{k}\right|^{2}+\tau_{k} u_{k}^{2}\right) d v_{g}=\int_{U_{i}}\left(\left|\nabla_{g} u_{\infty}\right|^{2}+\tau_{\infty} u_{\infty}^{2}\right) d v_{g}+4 \pi I^{(i)},
$$

where $U_{i}$ is a neighborhood of $x_{i}^{*}$ as above.

In the coordinate system $\left(U_{i}, \phi_{i} ;\left\{x^{1}, x^{2}\right\}\right)$, we write $\widetilde{x}_{i, k}=\phi_{i}^{-1}\left(x_{i, k}\right), \widetilde{u}_{k}(x)=u_{k}\left(\phi_{i}^{-1}(x)\right)$, $\widetilde{\tau}_{k}(x)=\tau_{k}\left(\phi_{i}^{-1}(x)\right)$ and $\widetilde{f}_{k}\left(x, \widetilde{u}_{k}(x)\right)=f_{k}\left(\phi_{i}^{-1}(x), u_{k}\left(\phi_{i}^{-1}(x)\right)\right)$ for any $x \in \Omega$. Moreover for $0<s<t<\delta$ we define the spherical mean of $\widetilde{u}_{k}$, the total energy and the neck energy of $\widetilde{u}_{k}$ around $\widetilde{x}_{i, k}$ by

$$
\varphi_{k}(t)=\varphi_{k}^{(i)}(t)=\frac{1}{2 \pi t} \int_{\partial \mathbb{B}_{t}\left(\widetilde{x}_{i, k}\right)} \widetilde{u}_{k} d \sigma
$$




$$
\Lambda_{k}(t)=\Lambda_{k}^{(i)}(t)=\int_{\mathbb{B}_{t}\left(\widetilde{x}_{i, k}\right)} \widetilde{u}_{k} \widetilde{f}_{k}\left(x, \widetilde{u}_{k}\right) d x
$$

and

$$
N_{k}(s, t)=N_{k}^{(i)}(s, t)=\int_{\mathbb{B}_{t}\left(\widetilde{x}_{i, k}\right) \backslash \mathbb{B}_{s}\left(\widetilde{x}_{i, k}\right)} \widetilde{u}_{k} \widetilde{f_{k}}\left(x, \widetilde{u}_{k}\right) d x
$$

respectively. We say that the property $\left(\mathcal{H}_{\ell}\right)$ holds if there exist sequences

$$
s_{k}^{(0)}=0<r_{k}^{(1)}<s_{k}^{(1)}<\cdots<r_{k}^{(\ell)}<s_{k}^{(\ell)}=o(1)
$$

such that the following hypotheses are satisfied:

$\left(\mathcal{H}_{\ell, 1}\right) \lim _{k \rightarrow \infty} r_{k}^{(j)} / s_{k}^{(j)}=\lim _{k \rightarrow \infty} s_{k}^{(j-1)} / r_{k}^{(j)}=0$ for all $1 \leq j \leq \ell ;$

$\left(\mathcal{H}_{\ell, 2}\right) \lim _{k \rightarrow \infty} \varphi_{k}\left(s_{k}^{(j)}\right) / \varphi_{k}\left(L r_{k}^{(j)}\right)=0$ for all $1 \leq j \leq \ell$ and all $L>0$;

$\left(\mathcal{H}_{\ell, 3}\right) \quad \lim _{k \rightarrow \infty} \Lambda_{k}\left(s_{k}^{(j)}\right)=4 \pi j$ for all $1 \leq j \leq \ell$;

$\left(\mathcal{H}_{\ell, 4}\right) \lim _{L \rightarrow \infty} \lim _{k \rightarrow \infty}\left(N_{k}\left(s_{k}^{(j-1)}, r_{k}^{(j)} / L\right)+N_{k}\left(L r_{k}^{(j)}, s_{k}^{(j)}\right)\right)=0$ for all $1 \leq j \leq \ell$.

To prove Proposition 5.1, we follow the lines of [8, 10, 13]. Precisely we use induction as follows: $\left(\mathcal{H}_{1}\right)$ holds; if $\left(\mathcal{H}_{\ell}\right)$ holds, then either $\left(\mathcal{H}_{\ell+1}\right)$ holds, or

$$
\lim _{L \rightarrow \infty} \lim _{k \rightarrow \infty} N_{k}\left(s_{k}^{(\ell)}, \delta / L\right)=0 .
$$

In view of (5.3), we have

$$
\begin{aligned}
\Lambda_{k}\left(s_{k}^{(\ell)}\right) & =\int_{\mathbb{B}_{s_{k}^{(\ell)}}\left(\widetilde{x}_{i, k}\right)} \widetilde{u}_{k}(x) \widetilde{f}_{k}\left(x, \widetilde{u}_{k}(x)\right) d x \\
& =(1+o(1)) \int_{\mathbb{B}_{s_{(\ell)}\left(\widetilde{x}_{i, k}\right)}} \widetilde{u}_{k}(x) \widetilde{f}_{k}\left(x, \widetilde{u}_{k}(x)\right) e^{\psi_{i}(x)} d x \\
& =(1+o(1)) \int_{\phi_{i}^{-1}\left(\mathbb{B}_{s_{k}^{(\ell)}}\left(\widetilde{x}_{i, k}\right)\right)} u_{k} f_{k}\left(x, u_{k}\right) d v_{g} \\
& \leq(1+o(1)) \int_{\Sigma} u_{k} f_{k}\left(x, u_{k}\right) d v_{g} .
\end{aligned}
$$

This together with $(2.3)$ and $\left(\mathcal{H}_{\ell, 3}\right)$ implies that the induction terminates after finitely-many steps. Letting $\ell_{0}$ be the largest integer such that $\left(\mathcal{H}_{\ell_{0}}\right)$ holds. Since $\widetilde{x}_{i, k} \rightarrow 0$ as $k \rightarrow \infty$, in view of the last assertion of Proposition 3.1, for any fixed $L>2 / \delta$,

$$
\lim _{k \rightarrow \infty}\left\|\widetilde{u}_{k}-\widetilde{u}_{\infty}\right\|_{C^{1}\left(\Omega \backslash \mathbb{B}_{\delta / L}\left(\widetilde{x}_{i, k}\right)\right)}=0 .
$$

Moreover it follows from $\left(\mathcal{H}_{\ell_{0}, 3}\right)$ and (5.5) (with $\ell$ replaced by $\ell_{0}$ ) that

$$
\lim _{L \rightarrow \infty} \lim _{k \rightarrow \infty} \int_{\phi_{i}^{-1}\left(\mathbb{B}_{\delta / L}\left(\widetilde{x}_{i, k}\right)\right)} u_{k} f_{k}\left(x, u_{k}\right) d v_{g}=\lim _{L \rightarrow \infty} \lim _{k \rightarrow \infty} \int_{\mathbb{B}_{\delta / L}\left(\widetilde{x}_{i, k}\right)} \widetilde{u}_{k} \widetilde{f}_{k}\left(x, \widetilde{u}_{k}\right) d x=4 \pi \ell_{0} .
$$

Recalling equation (1.2), we obtain (5.1) by combining (5.6) and (5.7) with $I^{(i)}=\ell_{0}$, and thus complete the proof of Proposition 5.1. 
The proof of the above induction process will be divided into the two steps below.

Step 1. The property $\left(\mathcal{H}_{1}\right)$ holds.

For any function $h: \Omega=\phi_{i}\left(U_{i}\right) \rightarrow \mathbb{R}$, denote the spherical average of $h$ around $\widetilde{x}_{i, k}$ by

$$
\bar{h}(r)=\frac{1}{2 \pi r} \int_{\partial \mathbb{B}_{r}\left(\widetilde{x}_{i, k}\right)} h d \sigma, \quad \forall 0<r<\delta .
$$

Let $w_{k}$ be the unscaled function with respect to the blow-up sequence $\eta_{i, k}$ as in (3.4), namely

$$
w_{k}(x)=u_{k}\left(x_{i, k}\right)\left(\widetilde{u}_{k}(x)-u_{k}\left(x_{i, k}\right)\right), \quad x \in \Omega .
$$

The decay estimate on $\bar{w}_{k}$ near the point $\widetilde{x}_{i, k}$ is crucial for the property $\left(\mathcal{H}_{1}\right)$. Precisely we have the following result.

Lemma 5.2 Given $0<\epsilon<1$. Let $T_{k}$ be the smallest number such that $\varphi_{k}\left(T_{k}\right)=\epsilon u_{k}\left(x_{i, k}\right)$. Then $r_{i, k} / T_{k} \rightarrow 0$ as $k \rightarrow \infty$, where $r_{i, k}$ is as in (3.3). Moreover, for any $b<2$, there exist some integer $k_{0}$ and a constant $C$ such that when $k \geq k_{0}$, we have

$$
\bar{w}_{k}(r) \leq b \log \frac{r_{i, k}}{r}+C
$$

for all $0 \leq r \leq T_{k}$ and

$$
\lim _{k \rightarrow \infty} \Lambda_{k}\left(T_{k}\right)=4 \pi .
$$

Proof. It follows from Proposition 3.1 and the definition of $T_{k}$ that $r_{i, k}=o\left(T_{k}\right)$ as $k \rightarrow \infty$. In view of $[1.2), \widetilde{u}_{k}$ satisfies the equation

$$
-\Delta_{\mathbb{R}^{2}} \widetilde{u}_{k}=e^{\psi_{i}}\left(\widetilde{f}_{k}\left(x, \widetilde{u}_{k}\right)-\widetilde{\tau}_{k} \widetilde{u}_{k}\right) \quad \text { in } \quad \Omega .
$$

Let $\left(v_{k}\right)$ be a sequence of solutions to

$$
\left\{\begin{array}{lll}
-\Delta_{\mathbb{R}^{2}} v_{k}=e^{\psi_{i}} \widetilde{f}_{k}\left(x, \widetilde{u}_{k}\right) & \text { on } & \mathbb{B}_{T_{k}}\left(\widetilde{x}_{i, k}\right) \\
v_{k}=\widetilde{u}_{k} & \text { on } & \partial \mathbb{B}_{T_{k}}\left(\widetilde{x}_{i, k}\right) .
\end{array}\right.
$$

Then we have by (5.10)

$$
\left\{\begin{array}{lll}
-\Delta_{\mathbb{R}^{2}}\left(v_{k}-\widetilde{u}_{k}\right)=e^{\psi_{i}} \widetilde{\tau}_{k} \widetilde{u}_{k} & \text { on } & \mathbb{B}_{T_{k}}\left(\widetilde{x}_{i, k}\right) \\
v_{k}-\widetilde{u}_{k}=0 & \text { on } & \partial \mathbb{B}_{T_{k}}\left(\widetilde{x}_{i, k}\right) .
\end{array}\right.
$$

Applying elliptic estimates to (5.12), we can find some constant $C$ independent of $k$ such that

$$
\left|v_{k}(x)-\widetilde{u}_{k}(x)\right| \leq C \text { for all } x \in \mathbb{B}_{T_{k}}\left(\widetilde{x}_{i, k}\right) .
$$

Moreover, it follows from Proposition 4.1 that

$$
\inf _{\partial \mathbb{B}_{T_{k}}\left(\widetilde{x}_{i, k}\right)} \widetilde{u}_{k} \geq \varphi_{k}\left(T_{k}\right)-C
$$


for some constant $C$ depending only on the Riemannian metric $g$. Applying the maximum principle to 5.11 , we have by 5.13 .

$$
\widetilde{u}_{k}(x) \geq \varphi_{k}\left(T_{k}\right)-C \text { for all } x \in \mathbb{B}_{T_{k}}\left(\widetilde{x}_{i, k}\right) .
$$

Note that $\varphi_{k}\left(T_{k}\right)=\epsilon u_{k}\left(x_{i, k}\right)$. For any $0 \leq t \leq T_{k}$, we have by (5.14) and the fact that $u_{k} \rightarrow u_{\infty}$ strongly in $L^{2}(\Sigma)$

$$
u_{k}\left(x_{i, k}\right) \int_{\mathbb{B}_{t}\left(\widetilde{x}_{i, k}\right)} e^{\psi_{i}} \widetilde{\tau}_{k} \widetilde{u}_{k} d x \leq \frac{\left\|\tau_{k}\right\|_{L^{\infty}(\Sigma)}}{\epsilon} \int_{\mathbb{B}_{t}\left(\widetilde{x}_{i, k}\right)} e^{\psi_{i}}\left(\widetilde{u}_{k}^{2}+C \widetilde{u}_{k}\right) d x=o(1) .
$$

For any $L r_{i, k} \leq t \leq T_{k}$, we obtain by Proposition 3.1

$$
\begin{aligned}
-u_{k}\left(x_{i, k}\right) \int_{\mathbb{B}_{t}\left(\widetilde{x}_{i, k}\right)} e^{\psi_{i}} \widetilde{f_{k}}\left(x, \widetilde{u}_{k}\right) d x & \leq-u_{k}\left(x_{i, k}\right) \int_{\mathbb{B}_{L_{r, k}}\left(\widetilde{x}_{i, k}\right)} e^{\psi_{i}} \widetilde{f}_{k}\left(x, \widetilde{u}_{k}\right) d x \\
& =-r_{i, k}^{-2} \int_{\mathbb{B}_{L_{r_{i}, k}}\left(\widetilde{x}_{i, k}\right)} e^{\psi_{i}} \frac{\widetilde{f_{k}}\left(x, \widetilde{u}_{k}\right)}{f_{k}\left(x_{i, k}, u_{k}\left(x_{i, k}\right)\right)} d x \\
& =-(1+o(1)) \int_{\mathbb{B}_{L}(0)} e^{(2+o(1)) \eta_{\infty}} d x \\
& =-4 \pi+o(1),
\end{aligned}
$$

where $o(1) \rightarrow 0$ as $k \rightarrow \infty$ first, and then $L \rightarrow \infty$. In view of (5.10), $\bar{w}_{k}$ satisfies

$$
-\Delta_{\mathbb{R}^{2}} \bar{w}_{k}=u_{k}\left(x_{i, k}\right) \overline{e^{\psi_{i}} \widetilde{f}_{k}\left(x, \widetilde{u}_{k}\right)}-u_{k}\left(x_{i, k}\right) \overline{e^{\psi_{i}} \widetilde{\tau}_{k} \widetilde{u}_{k}} .
$$

Then we have for any $L r_{i, k} \leq t \leq T_{k}$

$$
\begin{aligned}
& 2 \pi t \bar{w}_{k}^{\prime}(t)=\int_{\partial \mathbb{B}_{t}\left(\widetilde{x}_{i, k}\right)} \partial_{\nu} \bar{w}_{k} d \sigma=\int_{\mathbb{B}_{r}\left(\widetilde{x}_{i, k}\right)} \Delta_{\mathbb{R}^{2} \bar{w}_{k}} d x \\
& =-u_{k}\left(x_{i, k}\right) \int_{\mathbb{B}_{t}\left(\widetilde{x}_{i k}\right)} \overline{e^{\psi_{i}} \widetilde{f}_{k}\left(x, \widetilde{u}_{k}\right)} d x+u_{k}\left(x_{i, k}\right) \int_{\mathbb{B}_{l}\left(\widetilde{x}_{i, k}\right)} \overline{e^{\psi_{i}} \widetilde{\tau}_{k} \widetilde{u}_{k}} d x \\
& =-u_{k}\left(x_{i, k}\right) \int_{\mathbb{B}_{t}\left(\widetilde{x}_{i, k}\right)} e^{\psi_{i}} \widetilde{f}_{k}\left(x, \widetilde{u}_{k}\right) d x+u_{k}\left(x_{i, k}\right) \int_{\mathbb{B}_{t}\left(\widetilde{x}_{i, k}\right)} e^{\psi_{i}} \widetilde{\tau}_{k} \widetilde{u}_{k} d x \\
& \leq-4 \pi+o(1) .
\end{aligned}
$$

Here we used (5.15) and (5.16) in the last inequality. Thus for any $b<2$, there exists some integer $k_{0}$ such that

$$
\bar{w}_{k}^{\prime}(t) \leq-\frac{b}{t} \text { for all } k \geq k_{0} .
$$

This together with Proposition 3.1 leads to

$$
\begin{aligned}
& \bar{w}_{k}(t) \leq \bar{w}_{k}\left(L r_{i, k}\right)-b \log \frac{t}{L r_{i, k}} \\
& \leq \log \frac{1}{1+L^{2}}-b \log \frac{t}{L r_{i, k}}+o(1) \\
& \leq b \log \frac{r_{i, k}}{t}+C \\
& 21
\end{aligned}
$$


for some constant $C$, all $k \geq k_{0}$, and all $L r_{i, j} \leq t \leq T_{k}$. It follows from Proposition 3.1 again that the above inequality also holds for $0 \leq t \leq L r_{i, k}$. Hence (5.8) holds.

By (5.8) and (5.14) we have

$$
(\epsilon-1) u_{k}^{2}\left(x_{i, k}\right)-C u_{k}\left(x_{i, k}\right) \leq \bar{w}_{k}(r) \leq C, \quad \forall r \in\left[L r_{i, k}, T_{k}\right] .
$$

Hence there holds for $L r_{i, k} \leq r \leq T_{k}$

$$
\begin{aligned}
\varphi_{k}^{2}(r)-u_{k}^{2}\left(x_{i, k}\right) & =\left(1+\frac{\varphi_{k}(r)}{u_{k}\left(x_{i, k}\right)}\right) \bar{w}_{k}(r) \\
& =\left(2+\frac{\bar{w}_{k}}{u_{k}^{2}\left(x_{i, k}\right)}\right) \bar{w}_{k}(r) \\
& \leq(1+\epsilon+o(1)) \bar{w}_{k}(r)+(1-\epsilon+o(1)) C \\
& \leq(1+2 \epsilon / 3) b \log \frac{r_{i, k}}{r}+C,
\end{aligned}
$$

provided that $k$ is sufficiently large. For $0<r<\delta$ we denote

$$
\theta_{k}(r)=\theta_{k}^{(i)}(r)=\frac{1}{2 \pi r} \int_{\partial \mathbb{B}_{r}\left(\widetilde{x}_{i, k}\right)} \widetilde{f}_{k}\left(x, \varphi_{k}(r)\right) d \sigma .
$$

Taking $b$ such that $(1+2 \epsilon / 3) b=2+\epsilon$ in (5.17) and recalling (H4) and (H5), we can find some constant $C$ such that for $L r_{i, k} \leq r \leq T_{k}$

$$
\begin{aligned}
\frac{\theta_{k}(r)}{f_{k}\left(x_{i, k}, u_{k}\left(x_{i, k}\right)\right)} & =\frac{\theta_{k}(r)}{\widetilde{f_{k}}\left(\widetilde{x}_{i, k}, \varphi_{k}(r)\right)} \frac{\widetilde{f}_{k}\left(\widetilde{x}_{i, k}, \varphi_{k}(r)\right)}{f_{k}\left(x_{i, k}, u_{k}\left(x_{i, k}\right)\right)} \\
& =(1+o(1)) \frac{f_{k}\left(x_{i, k}, \varphi_{k}(r)\right)}{f_{k}\left(x_{i, k}, u_{k}\left(x_{i, k}\right)\right)} \\
& =(1+o(1)) e^{(1+o(1))\left(\varphi_{k}^{2}(r)-u_{k}^{2}\left(x_{i, k}\right)\right)} \\
& \leq C\left(\frac{r_{i, k}}{r}\right)^{2+\epsilon}
\end{aligned}
$$

for sufficiently large $k$. For $0<s<t<\delta$, we define next a function analogous to (5.4) as below.

$$
\bar{N}_{k}(s, t)=\bar{N}_{k}^{(i)}(s, t)=2 \pi \int_{s}^{t} r \varphi_{k}(r) \theta_{k}(r) d r .
$$

In view of (5.8) and (5.19), we estimate

$$
\begin{aligned}
\bar{N}_{k}\left(L r_{i, k}, T_{k}\right) & =2 \pi \int_{L r_{i, k}}^{T_{k}} r \varphi_{k}(r) \theta_{k}(r) d r \\
& =2 \pi r_{i, k}^{-2} \int_{L r_{i, k}}^{T_{k}} r \frac{\varphi_{k}(r)}{u_{k}\left(x_{i, k}\right)} \frac{\theta_{k}(r)}{f_{k}\left(x_{i, k}, u_{k}\left(x_{i, k}\right)\right)} d r \\
& \leq 2 \pi(1+o(1)) C r_{i, k}^{\epsilon} \int_{L r_{i, k}}^{T_{k}} \frac{1}{r^{1+\epsilon}} d r \\
& \leq 2 \pi(1+o(1)) C \epsilon^{-1} L^{-\epsilon} .
\end{aligned}
$$


This leads to

$$
\lim _{L \rightarrow \infty} \lim _{k \rightarrow \infty} \bar{N}_{k}\left(L r_{i, k}, T_{k}\right)=0 .
$$

Since Proposition 4.1 implies that

$$
u_{k}^{2}(x)-\varphi_{k}^{2}(r) \leq C \quad \text { for all } \quad x \in \partial \mathbb{B}_{r}\left(\widetilde{x}_{i, k}\right),
$$

there holds

$$
N_{k}\left(L r_{i, k}, T_{k}\right) \leq C \bar{N}_{k}\left(L r_{i, k}, T_{k}\right)+o(1) .
$$

This together with 5.21) leads to

$$
\lim _{L \rightarrow \infty} \lim _{k \rightarrow \infty} N_{k}\left(L r_{i, k}, T_{k}\right)=0 .
$$

By Proposition 3.1,

$$
\Lambda_{k}\left(L r_{i, k}\right)=\int_{\left.\mathbb{B}_{L r_{i, k}} \widetilde{x}_{i, k}\right)} \widetilde{u}_{k} \widetilde{f}_{k}\left(x, \widetilde{u}_{k}\right) d x=(1+o(1)) \int_{\mathbb{B}_{L}(0)} e^{2 \eta_{\infty}} d x .
$$

Hence

$$
\lim _{L \rightarrow \infty} \lim _{k \rightarrow \infty} \Lambda_{k}\left(L r_{i, k}\right)=4 \pi .
$$

Thus (5.9) follows immediately from (5.22) and (5.23).

By Lemma 5.2 we may choose a subsequence $u_{k}$, numbers $\epsilon_{k} \searrow 0$ as $k \rightarrow \infty$ and $s_{k}=T_{k}\left(\epsilon_{k}\right)$ with $r_{i, k} / s_{k} \rightarrow 0, \varphi_{k}\left(s_{k}\right) \rightarrow \infty$ as $k \rightarrow \infty$ and such that

$$
\lim _{k \rightarrow \infty} \Lambda_{k}\left(s_{k}\right)=4 \pi, \quad \lim _{L \rightarrow \infty} \lim _{k \rightarrow \infty} N_{k}\left(L r_{i, k}, s_{k}\right)=0,
$$

while in addition

$$
\lim _{k \rightarrow \infty} \frac{\varphi_{k}\left(s_{k}\right)}{\varphi_{k}\left(L r_{i, k}\right)}=0, \quad \forall L>0 .
$$

Let $r_{k}^{(1)}=r_{i, k}, s_{k}^{(1)}=s_{k}$. Then $\left(\mathcal{H}_{1}\right)$ holds and Step 1 is finished.

Step 2. Suppose that $\left(\mathcal{H}_{\ell}\right)$ already holds for some integer $\ell \geq 1$, namely there exist sequences $s_{k}^{(0)}=0<r_{k}^{(1)}<s_{k}^{(1)}<\cdots<r_{k}^{(\ell)}<s_{k}^{(\ell)}=o(1)$ such that $\left(\mathcal{H}_{\ell, 1}\right)$ up to $\left(\mathcal{H}_{\ell, 4}\right)$ hold. Then we shall prove that either $\lim _{L \rightarrow \infty} \lim _{k \rightarrow \infty} N_{k}\left(s_{k}^{(\ell)}, \delta / L\right)=0$ or $\left(\mathcal{H}_{\ell+1}\right)$ holds.

Setting

$$
P_{k}(t)=P_{k}^{(i)}(t)=t \int_{\partial \mathbb{B}_{t}} \widetilde{u}_{k} \widetilde{f}_{k}\left(x, \widetilde{u}_{k}\right) d \sigma, \quad \bar{P}_{k}(t)=\bar{P}_{k}^{(i)}(t)=2 \pi t^{2} \varphi_{k}(t) \theta_{k}(t)
$$

and assuming $\left(\mathcal{H}_{\ell}\right)$ holds, we have the following result.

Lemma 5.3 There exists a constant $C_{0}$ depending only on the upper bound of the total energy (2.3) and the Riemannian metric $g$ such that for $s_{k}^{(\ell)} \leq t_{k}=o(1)$, there holds

$$
\bar{N}_{k}\left(s_{k}^{(\ell)}, t_{k}\right) \leq \bar{P}_{k}\left(t_{k}\right)+C_{0} \bar{N}_{k}^{2}\left(s_{k}^{(\ell)}, t_{k}\right)+o(1),
$$


where $o(1) \rightarrow 0$ as $k \rightarrow \infty, \bar{N}_{k}$ and $\bar{P}_{k}$ are defined as in (5.20) and (5.24) respectively.

Proof. We first claim that there exists a constant $C$ depending only on $\delta$ and the Riemannian metric $g$ such that

$$
\varphi_{k}(s) \leq \sup _{\partial \mathbb{B}_{s}\left(\widetilde{x}_{i, k}\right)} \widetilde{u}_{k} \leq \inf _{\partial \mathbb{B}_{r}\left(\widetilde{x}_{i, k}\right)} \widetilde{u}_{k}+C \leq \varphi_{k}(r)+C \text { for all } 0<r<s \leq \delta .
$$

To see the last inequality, we set $v_{k}$ be a positive solution of

$$
\left\{\begin{array}{lll}
-\Delta_{\mathbb{R}^{2}} v_{k}=e^{\psi_{i}} \widetilde{f}_{k}\left(x, \widetilde{u}_{k}\right) & \text { in } & \mathbb{B}_{\delta}\left(\widetilde{x}_{i, k}\right) \\
v_{k}=\widetilde{u}_{k} & \text { on } & \partial \mathbb{B}_{\delta}\left(\widetilde{x}_{i, k}\right) .
\end{array}\right.
$$

Thus we have by (1.2)

$$
\left\{\begin{array}{lll}
-\Delta_{\mathbb{R}^{2}}\left(v_{k}-\widetilde{u}_{k}\right)=e^{\psi} \widetilde{\tau}_{k} \widetilde{u}_{k} & \text { in } & \mathbb{B}_{\delta}\left(\widetilde{x}_{i, k}\right) \\
v_{k}-\widetilde{u}_{k}=0 & \text { on } & \partial \mathbb{B}_{\delta}\left(\widetilde{x}_{i, k}\right) .
\end{array}\right.
$$

Noting that $\left\|e^{\psi_{i}} \widetilde{\tau}_{k} \widetilde{u}_{k}\right\|_{L^{p}\left(\mathbb{B}_{\delta}\left(\widetilde{x}_{i, k}\right)\right)}$ is bounded for any $p>1$ and applying elliptic regularity estimates to 5.28 , we then find some constant $C=C(\delta)$ such that

$$
v_{k}(x)-C \leq \widetilde{u}_{k}(x) \leq v_{k}(x)+C \text { for all } x \in \mathbb{B}_{\delta}\left(\widetilde{x}_{i, k}\right) .
$$

By (5.27), we have for $0<r<\delta$

$$
-\left(r \bar{v}_{k}^{\prime}(r)\right)^{\prime}=r \overline{e^{\psi_{i}} \widetilde{f}_{k}\left(x, \widetilde{u}_{k}\right)} .
$$

Integration from 0 to $r$ gives

$$
-r \bar{v}_{k}^{\prime}(r)=\int_{0}^{r} r \overline{e^{\psi_{i}} \widetilde{f}_{k}\left(x, \widetilde{u}_{k}\right)} d r .
$$

Hence

$$
\bar{v}_{k}^{\prime}(r) \leq 0 \text { for all } 0<r<\delta .
$$

Now fix $0<r<s \leq \delta$. There exist two points $\xi \in \partial \mathbb{B}_{r}\left(\widetilde{x}_{i, k}\right)$ and $\zeta \in \partial \mathbb{B}_{s}\left(\widetilde{x}_{i, k}\right)$ such that

$$
v_{k}(\xi)=\bar{v}_{k}(r), \quad v_{k}(\zeta)=\bar{v}_{k}(s) .
$$

This together with the gradient estimate (Proposition 4.1), 5.29), and (5.30) leads to

$$
\begin{aligned}
\sup _{\partial \mathbb{B}_{s}\left(\widetilde{x}_{i, k}\right)} \widetilde{u}_{k} & \leq \widetilde{u}_{k}(\zeta)+C \leq v_{k}(\zeta)+C \\
& \leq v_{k}(\xi)+C \leq \inf _{\partial \mathbb{B}_{r}\left(\widetilde{x}_{i, k}\right)} \widetilde{u}_{k}+C .
\end{aligned}
$$

This confirms our claim (5.26).

Next we calculate

$$
\begin{aligned}
\theta_{k}^{\prime}(r)= & \frac{d}{d r}\left(\frac{1}{2 \pi} \int_{0}^{2 \pi} \widetilde{f}_{k}\left(\widetilde{x}_{i, k}^{1}+r \cos \theta, \widetilde{x}_{i, k}^{2}+r \sin \theta, \varphi_{k}(r)\right) d \theta\right) \\
= & \frac{1}{2 \pi} \int_{0}^{2 \pi} \nabla_{x} \widetilde{f}_{k}\left(\widetilde{x}_{i, k}^{1}+r \cos \theta, \widetilde{x}_{i, k}^{2}+r \sin \theta, \varphi_{k}(r)\right) \cdot(\cos \theta, \sin \theta) d \theta \\
& +\frac{1}{2 \pi} \int_{0}^{2 \pi} \widetilde{f_{k}^{\prime}}\left(\widetilde{x}_{i, k}^{1}+r \cos \theta, \widetilde{x}_{i, k}^{2}+r \sin \theta, \varphi_{k}(r)\right) \varphi_{k}^{\prime}(r) d \theta,
\end{aligned}
$$


where we write $\widetilde{x}_{i, k}=\left(\widetilde{x}_{i, k}^{1}, \widetilde{x}_{i, k}^{2}\right)$. In view of (H4), we obtain

$$
\left|\theta_{k}^{\prime}(r)\right| \leq C\left(1+\theta_{k}(r)+\varphi_{k}(r)\left|\varphi_{k}^{\prime}(r)\right| \theta_{k}(r)\right) .
$$

For $s=s_{k}^{(\ell)} \leq t \leq t_{k}$, we have by equation (1.2).

$$
\begin{aligned}
-2 \pi t \varphi_{k}^{\prime}(t) & =-\int_{\partial \mathbb{B}_{t}\left(\widetilde{x}_{i, k}\right)} \partial_{\nu} \varphi_{k} d \sigma=-\int_{\mathbb{B}_{t}\left(\widetilde{x}_{i, k}\right)} \Delta_{\mathbb{R}^{2}} \varphi_{k} d x \\
& =\int_{\mathbb{B}_{t}\left(\widetilde{x}_{i, k}\right)} \overline{e^{\psi_{i}} \widetilde{f}_{k}\left(x, \widetilde{u}_{k}\right)} d x-\int_{\mathbb{B}_{t}\left(\widetilde{x}_{i, k}\right)} \overline{e^{\psi_{i}} \widetilde{\tau}_{k} \widetilde{u}_{k}} d x \\
& =\int_{\mathbb{B}_{t}\left(\widetilde{x}_{i, k}\right)} e^{\psi_{i}} \widetilde{f_{k}}\left(x, \widetilde{u}_{k}\right) d x-\int_{\mathbb{B}_{t}\left(\widetilde{x}_{i, k}\right)} e^{\psi_{i}} \widetilde{\tau}_{k} \widetilde{u}_{k} d x .
\end{aligned}
$$

It follows from (H4), (H5) and Proposition 4.1 that $\widetilde{f}_{k}\left(x, \widetilde{u}_{k}\right) \leq C\left(1+\widetilde{f}_{k}\left(x, \varphi_{k}(r)\right)\right) \leq C\left(1+\theta_{k}(r)\right)$, where $r=\left|x-\widetilde{x}_{i, k}\right|$. Combining (H4), (H5) and (5.26), we have

$$
\int_{\mathbb{B}_{s}\left(\widetilde{x_{k}}\right)} \varphi_{k}(s) \theta_{k}(r) d x \leq C\left(1+\Lambda_{k}(s)\right)
$$

where we used $r=\left|x-\widetilde{x}_{i, k}\right|$. Note that $\varphi_{k}(s) \rightarrow \infty$ as $k \rightarrow \infty$. We then obtain

$$
\begin{aligned}
-2 \pi t \varphi_{k}^{\prime}(t) & \leq C \int_{\mathbb{B}_{t}\left(\widetilde{x}_{i, k}\right)}\left(1+\theta_{k}(r)\right) d x \\
& \leq C \int_{\mathbb{B}_{t}\left(\widetilde{x}_{i, k}\right)\left(\mathbb{B}_{s}\left(\widetilde{x}_{i, k}\right)\right.} \theta_{k}(r) d x+\frac{C}{\varphi_{k}(s)} \int_{\mathbb{B}_{s}\left(\widetilde{x}_{i, k}\right)} \varphi_{k}(s) \theta_{k}(r) d x+o(1) \\
& \leq C \bar{N}_{k}(s, t)+o(1) .
\end{aligned}
$$

This immediately leads to

$$
-\pi \int_{s}^{t} r^{2} \varphi_{k}^{\prime}(r) \theta_{k}(r) d r \leq C \bar{N}_{k}^{2}(s, t)+o(1) .
$$

Similarly we have

$$
\begin{aligned}
-2 \pi t \varphi_{k}(t) \varphi_{k}^{\prime}(t) & =-\int_{\partial \mathbb{B}_{t}\left(\widetilde{x}_{i, k}\right)} \varphi_{k}(t) \partial_{\nu} \varphi_{k} d \sigma=-\int_{\mathbb{B}_{t}\left(\widetilde{x}_{i, k}\right)} \varphi_{k}(t) \Delta_{\mathbb{R}^{2}} \varphi_{k} d x \\
& =\int_{\mathbb{B}_{t}\left(\widetilde{x}_{i, k}\right)} \varphi_{k}(t) \overline{e^{\psi_{i}} \widetilde{f}_{k}\left(x, \widetilde{u}_{k}\right)} d x-\int_{\mathbb{B}_{t}\left(\widetilde{x}_{i, k}\right)} \varphi_{k}(t) \overline{e^{4} \widetilde{\tau}_{k} \widetilde{u}_{k}} d x \\
& =\int_{\mathbb{B}_{t}\left(\widetilde{x}_{i, k}\right)} \varphi_{k}(t) \overline{e^{\psi_{i}} \widetilde{f_{k}}\left(x, \widetilde{u}_{k}\right)} d x+o(1),
\end{aligned}
$$

where the last equality follows from (5.26) and $u_{k} \rightarrow u_{\infty}$ strongly in $L^{2}(\Sigma)$. Repeatedly using 
(5.26), we obtain

$$
\begin{aligned}
\int_{\mathbb{B}_{t}\left(\widetilde{x}_{i, k}\right)} \varphi(t) \overline{e^{\psi_{i}} \widetilde{f_{k}}\left(x, \widetilde{u}_{k}\right)} d x \leq & C \int_{\mathbb{B}_{t}\left(\widetilde{x}_{i, k}\right)} \varphi_{k}(t)\left(1+\theta_{k}(r)\right) d x \\
\leq & C \int_{\mathbb{B}_{t}\left(\widetilde{x}_{i, k}\right)\left(\mathbb{B}_{s}\left(\widetilde{x}_{i, k}\right)\right.}\left(1+\varphi_{k}(r)\right)\left(1+\theta_{k}(r)\right) d x \\
& +C \int_{\mathbb{B}_{s}\left(\widetilde{x}_{i, k}\right) \backslash \mathbb{B}_{L_{k}^{(r)}}\left(\widetilde{x}_{i, k}\right)}\left(1+\varphi_{k}(r)\right)\left(1+\theta_{k}(r)\right) d x \\
& +C \int_{\mathbb{B}_{L_{k}(\ell)\left(\widetilde{x}_{i, k}\right)}}\left(1+\varphi_{k}(s)\right)\left(1+\theta_{k}(r)\right) d x \\
\leq & C\left(\bar{N}_{k}(s, t)+\bar{N}_{k}\left(L r_{k}^{(\ell)}, s\right)+\frac{\varphi_{k}(s)}{\varphi_{k}\left(L r_{k}^{(\ell)}\right)}\left(\Lambda_{k}\left(L r_{k}^{(\ell)}\right)+o(1)\right)\right) .
\end{aligned}
$$

This together with $(5.33),\left(\mathcal{H}_{\ell, 2}\right)$ and $\left(\mathcal{H}_{\ell, 4}\right)$ implies

$$
2 \pi t \varphi_{k}(t)\left|\varphi_{k}^{\prime}(t)\right| \leq C \bar{N}_{k}(s, t)+o(1)
$$

Obviously

$$
\int_{s}^{t} r^{2} \varphi_{k}(r) d r=o(1), \quad \int_{s}^{t} r^{2} \varphi_{k}(r) \theta_{k}(r) d r=o(1) .
$$

It then follows from (5.31) and (5.34) that

$$
\begin{aligned}
-\pi \int_{s}^{t} r^{2} \varphi_{k}(r) \theta_{k}^{\prime}(r) d r & \leq \pi C \int_{s}^{t} r^{2} \varphi_{k}^{2}(r)\left|\varphi_{k}^{\prime}(r)\right| \theta_{k}(r) d r+o(1) \\
& \leq C \bar{N}_{k}^{2}(s, t)+o(1) .
\end{aligned}
$$

Integration by parts gives

$$
\begin{aligned}
\bar{N}_{k}(s, t) & =\int_{s}^{t} 2 \pi r \varphi_{k}(r) \theta_{k}(r) d r \\
& \leq \pi t^{2} \varphi_{k}(t) \theta_{k}(t)-\pi \int_{s}^{t} r^{2} \varphi_{k}^{\prime}(r) \theta_{k}(r) d r-\pi \int_{s}^{t} r^{2} \varphi_{k}(r) \theta_{k}^{\prime}(r) d r .
\end{aligned}
$$

This together with (5.32) and (5.35) implies (5.25).

Lemma 5.4 Let $C_{0}$ be the constant as in Lemma 5.3. Let $t_{k}$ be such that for a subsequence

$$
s_{k}^{(\ell)}<t_{k}=o(1), \quad 0<\lim _{k \rightarrow \infty} \bar{N}_{k}\left(s_{k}^{(\ell)}, t_{k}\right)=\alpha<\frac{1}{2 C_{0}} .
$$

Then $s_{k}^{(\ell)}=o\left(t_{k}\right)$ as $k \rightarrow \infty, \liminf _{k \rightarrow \infty} \bar{P}_{k}\left(t_{k}\right) \geq \alpha / 2$, and

$$
\lim _{L \rightarrow \infty} \lim _{k \rightarrow \infty} \bar{N}_{k}\left(s_{k}^{(\ell)}, t_{k} / L\right)=0,
$$

where $\bar{N}_{k}$ and $\bar{P}_{k}$ are as defined in (5.20) and (5.24) respectively. 
Proof. We first claim that

$$
\lim _{L \rightarrow \infty} \lim _{k \rightarrow \infty} \bar{N}_{k}\left(s_{k}^{(\ell)}, L s_{k}^{(\ell)}\right)=0 .
$$

Actually, in view of (5.26), we have for $0<t \leq t_{k}$

$$
\bar{P}_{k}(t) \leq C \bar{N}_{k}(t / 2, t)+o(1) \leq C \bar{P}_{k}(t / 2)+o(1),
$$

and

$$
\bar{N}_{k}(t, 2 t) \leq C \bar{N}_{k}(t / 2, t)+o(1) .
$$

In particular, for any $j \in \mathbb{N}$ there holds

$$
\begin{aligned}
\lim _{k \rightarrow \infty} \bar{N}_{k}\left(2^{j-1} s_{k}^{(\ell)}, 2^{j} s_{k}^{(\ell)}\right) & \leq C \lim _{k \rightarrow \infty} \bar{N}_{k}\left(2^{j-2} s_{k}^{(\ell)}, 2^{j-1} s_{k}^{(\ell)}\right) \\
& \leq C^{j} \lim _{k \rightarrow \infty} \bar{N}_{k}\left(s_{k}^{(\ell)} / 2, s_{k}^{(\ell)}\right)=0 .
\end{aligned}
$$

If $L \leq 2^{j}$, we obtain

$$
\lim _{k \rightarrow \infty} \bar{N}_{k}\left(s_{k}^{(\ell)}, L s_{k}^{(\ell)}\right) \leq \lim _{k \rightarrow \infty} \sum_{m=1}^{j} \bar{N}_{k}\left(2^{m-1} s_{k}^{(\ell)}, 2^{m} s_{k}^{(\ell)}\right)=0 .
$$

Thus our claim 5.37 follows immediately. One can see from 5.37) that $s_{k}^{(\ell)} / t_{k} \rightarrow 0$ as $k \rightarrow \infty$. By Lemma 5.3,

$$
\liminf _{k \rightarrow \infty} \bar{P}_{k}\left(t_{k}\right) \geq \frac{1}{2} \lim _{k \rightarrow \infty} \bar{N}_{k}\left(s_{k}^{(\ell)}, t_{k}\right)=\frac{\alpha}{2} .
$$

Now we show (5.36). Assuming the contrary, there holds

$$
\lim _{L \rightarrow \infty} \lim _{k \rightarrow \infty} \bar{N}_{k}\left(s_{k}^{(\ell)}, t_{k} / L\right)=\beta>0 .
$$

Then we have for any fixed $L \geq 1$ and all sufficiently large $k$

$$
\frac{\beta}{2} \leq \bar{N}_{k}\left(s_{k}^{(\ell)}, t_{k} / L\right) \leq \bar{N}_{k}\left(s_{k}^{(\ell)}, t_{k}\right)<\frac{1}{2 C_{0}} .
$$

Applying (5.25) with $t_{k} / L$ instead of $t_{k}$, we get

$$
\lim _{k \rightarrow \infty} \bar{P}_{k}\left(t_{k} / L\right) \geq \frac{\beta}{4}
$$

and then by

$$
C \lim _{k \rightarrow \infty} \bar{N}_{k}\left(t_{k} /(2 L), t_{k} / L\right) \geq \lim _{k \rightarrow \infty} \bar{P}_{k}\left(t_{k} / L\right) \geq \frac{\beta}{4} .
$$

Choosing $L=2^{m}, m=0,1, \cdots, j-1$, we have

$$
\frac{j \beta}{4} \leq C \lim _{k \rightarrow \infty} \bar{N}_{k}\left(2^{-j} t_{k}, t_{k}\right) \leq C\left(1+\limsup _{k \rightarrow \infty} \Lambda_{k}\left(t_{k}\right)\right) \leq C .
$$

We get a contradiction by letting $j \rightarrow \infty$ and obtain (5.36). 
Lemma 5.5 Suppose that

$$
\lim _{k \rightarrow \infty} \sup _{s_{k}^{(\ell)}<t<t_{k}} \bar{P}_{k}(t)=0 \quad \text { for any sequence } t_{k} \rightarrow 0 \text { as } k \rightarrow \infty .
$$

Then we have

$$
\lim _{L \rightarrow \infty} \lim _{k \rightarrow \infty} \bar{N}_{k}\left(s_{k}^{(\ell)}, \delta / L\right)=0 .
$$

Proof. In view of Lemma 5.4, it suffices to prove

$$
\lim _{L \rightarrow \infty} \lim _{k \rightarrow \infty} \sup _{s_{k}^{(\ell)}<t<\delta / L} \bar{P}_{k}(t)=0 .
$$

Indeed, if we take some number $t_{k, L} \in\left(s_{k}^{(\ell)}, \delta / L\right)$ such that

$$
\bar{P}_{k}\left(t_{k, L}\right)=\sup _{s_{k}^{(\ell)}<t<\delta / L} \bar{P}_{k}(t)
$$

then either

$$
\lim _{k \rightarrow \infty} t_{k, L}=0
$$

or

$$
\lim _{k \rightarrow \infty} t_{k, L}=t_{L}^{*}>0 .
$$

In case of (5.42), we already have (5.41) because of (5.40). While in case of (5.43), we have by using (5.26)

$$
\bar{P}_{k}\left(t_{k, L}\right) \leq C t_{k, L}^{2}\left(1+\varphi_{k}\left(t_{L}^{*} / 2\right) \theta_{k}\left(t_{L}^{*} / 2\right)\right)
$$

for sufficiently large $k$. Note that $\partial \mathbb{B}_{t_{L}^{*} / 2}\left(\widetilde{x}_{i, k}\right) \subset \mathbb{B}_{t_{L}^{*}}\left(\widetilde{x}_{i}^{*}\right) \backslash \mathbb{B}_{t_{L}^{*} / 3}\left(\widetilde{x}_{i}^{*}\right)$ for sufficiently large $k$, and that $t_{k, L} \leq \delta / L \rightarrow 0$ as $k \rightarrow \infty$ first and then $L \rightarrow \infty$. Moreover, by Proposition 3.1, we have $u_{k} \rightarrow u_{\infty}$ in $C_{\text {loc }}^{1}\left(\Sigma \backslash \cup_{j=1}^{N}\left\{x_{j}^{*}\right\}, \mathbb{R}\right)$ and $u_{\infty} \in C^{1}(\Sigma, \mathbb{R})$, In particular, $u_{\infty}$ is bounded on $\mathbb{B}_{\delta}\left(x_{i}^{*}\right)$. It then follows from (5.44) that

$$
\lim _{L \rightarrow \infty} \lim _{k \rightarrow \infty} \bar{P}_{k}\left(t_{k, L}\right)=0
$$

Thus (5.41) holds again.

If the assumption (5.40) is not satisfied, then (5.38) implies that there exists a sequence $t_{k} \rightarrow 0$ as $k \rightarrow \infty$ such that

$$
\lim _{k \rightarrow \infty} \bar{N}_{k}\left(s_{k}^{(\ell)}, t_{k}\right)>0 .
$$

We shall show that the property $\left(\mathcal{H}_{\ell+1}\right)$ holds. Take $r_{k}^{(\ell+1)} \in\left(s_{k}^{(\ell)}, t_{k}\right)$ such that up to a subsequence, there holds

$$
0<\lim _{k \rightarrow \infty} \bar{N}_{k}\left(s_{k}^{(\ell)}, r_{k}^{(\ell+1)}\right)<\frac{1}{2 C_{0}}
$$

where $C_{0}$ is as in Lemma 5.3. It then follows from Lemma 5.4 that

$$
\begin{gathered}
\lim _{k \rightarrow \infty} \frac{s_{k}^{(\ell)}}{r_{k}^{(\ell+1)}}=0, \quad \lim _{k \rightarrow \infty} \bar{N}_{k}\left(s_{k}^{(\ell)}, r_{k}^{(\ell+1)}\right)>0, \\
\liminf _{k \rightarrow \infty} \bar{P}_{k}\left(r_{k}^{(\ell+1)}\right)>0, \quad \lim _{k \rightarrow \infty} \varphi_{k}\left(r_{k}^{(\ell+1)}\right)=\infty,
\end{gathered}
$$


and that

$$
\lim _{L \rightarrow \infty} \lim _{k \rightarrow \infty} \bar{N}_{k}\left(s_{k}^{(\ell)}, r_{k}^{(\ell+1)} / L\right)=0 .
$$

Moreover, we have the following result.

Lemma 5.6 Up to a subsequence there holds

$$
\eta_{k}^{(\ell+1)}(x):=\varphi_{k}\left(r_{k}^{(\ell+1)}\right)\left(\widetilde{u}_{k}\left(\widetilde{x}_{i, k}+r_{k}^{(\ell+1)} x\right)-\varphi_{k}\left(r_{k}^{(\ell+1)}\right)\right) \rightarrow \eta^{(\ell+1)}(x)
$$

in $C_{\mathrm{loc}}^{1}\left(\mathbb{R}^{2} \backslash\{0\}\right)$ as $k \rightarrow \infty$, where

$$
\eta^{(\ell+1)}(x)=\log \frac{2}{\sqrt{\alpha_{0}}\left(1+|x|^{2}\right)}
$$

and

$$
\int_{\mathbb{R}^{2}} e^{2 \eta^{(\ell+1)}} d x=\frac{4 \pi}{\alpha_{0}}
$$

for some constant $\alpha_{0}>0$.

Proof. To simplify the notations we write $r_{k}=r_{k}^{(\ell+1)}, \eta_{k}=\eta_{k}^{(\ell+1)}$, and $\eta=\eta^{(\ell+1)}$. For any fixed $L>0$, we set

$$
v_{k}(x)=\widetilde{u}_{k}\left(\widetilde{x}_{i, k}+r_{k} x\right), \quad x \in \mathbb{B}_{L}(0) \backslash \mathbb{B}_{1 / L}(0) .
$$

In view of Proposition 4.1, there exists some constant $C=C(L)$ such that

$$
\left|\widetilde{u}_{k}^{2}\left(\widetilde{x}_{i, k}+r_{k} x\right)-\varphi_{k}^{2}\left(r_{k}\right)\right| \leq C
$$

and thus

$$
\left|\varphi_{k}\left(r_{k}\right)\left(\widetilde{u}_{k}\left(\widetilde{x}_{i, k}+r_{k} x\right)-\varphi_{k}\left(r_{k}\right)\right)\right| \leq C .
$$

Hence

$$
\eta_{k} \quad \text { is bounded in } L_{\mathrm{loc}}^{\infty}\left(\mathbb{R}^{2} \backslash\{0\}\right) .
$$

Combining (5.47) and (5.50), we have

$$
v_{k}-\varphi_{k}\left(r_{k}\right) \rightarrow 0 \quad \text { in } \quad L_{\mathrm{loc}}^{\infty}\left(\mathbb{R}^{2} \backslash\{0\}\right) \quad \text { as } \quad k \rightarrow \infty,
$$

in particular

$$
\frac{v_{k}}{\varphi_{k}\left(r_{k}\right)} \rightarrow 1 \quad \text { in } \quad L_{\mathrm{loc}}^{\infty}\left(\mathbb{R}^{2} \backslash\{0\}\right) \quad \text { as } \quad k \rightarrow \infty .
$$

By the equation (1.2), we write for $x \in \Omega_{k}=\left\{x \in \mathbb{R}^{2}: \widetilde{x}_{i, k}+r_{k} x \in \mathbb{B}_{\delta}(0)\right\}$

$$
-\Delta_{\mathbb{R}^{2}} \eta_{k}(x)=e^{\psi_{i}\left(\widetilde{x}_{i, k}+r_{k} x\right)} \varphi_{k}\left(r_{k}\right) r_{k}^{2} \widetilde{f}\left(\widetilde{x}_{i, k}+r_{k} x, v_{k}(x)\right)-e^{\psi_{i}\left(\widetilde{x}_{i, k}+r_{k} x\right)} \varphi_{k}\left(r_{k}\right) r_{k}^{2} \widetilde{\tau}_{k}\left(\widetilde{x}_{i, k}+r_{k} x\right) v_{k}(x) \text {. (5.53) }
$$

Since $u_{k} \rightarrow u_{\infty}$ strongly in $L^{2}(\Sigma)$, we have by using (5.52)

$$
\begin{aligned}
r_{k}^{2} \varphi_{k}^{2}\left(r_{k}\right) & =\frac{r_{k}^{2}}{3 \pi} \int_{\mathbb{B}_{2}(0) \backslash \mathbb{B}_{1}(0)} \varphi_{k}^{2}\left(r_{k}\right) d x \\
& =(1+o(1)) \frac{r_{k}^{2}}{3 \pi} \int_{\mathbb{B}_{2}(0)\left(\mathbb{B}_{1}(0)\right.} v_{k}^{2}(x) d x \\
& =\frac{1+o(1)}{3 \pi} \int_{\mathbb{B}_{2 r_{k}}\left(\widetilde{x}_{i, k}\right) \backslash \mathbb{B}_{r_{k}}\left(\widetilde{x}_{i, k}\right)} \widetilde{u}_{k}^{2}(y) d y \\
& \rightarrow \quad 0 \quad \text { as } \quad k \rightarrow \infty . \\
& 29
\end{aligned}
$$


By (5.47) we may assume

$$
r_{k}^{2} \varphi_{k}^{2}\left(r_{k}\right) \theta_{k}\left(r_{k}\right) \rightarrow \alpha_{0}>0
$$

Moreover, by (H4) and (H5) we have

$$
\begin{aligned}
\frac{\widetilde{f}_{k}\left(\widetilde{x}_{i, k}+r_{k} x, v_{k}(x)\right)}{\theta_{k}\left(r_{k}\right)} & =(1+o(1)) \frac{\widetilde{f}_{k}\left(\widetilde{x}_{i, k}+r_{k} x, v_{k}(x)\right)}{\widetilde{f}_{k}\left(\widetilde{x}_{i, k}+r_{k} x, \varphi_{k}\left(r_{k}\right)\right)} \\
& =(1+o(1)) e^{(1+o(1))\left(v_{k}^{2}(x)-\varphi_{i, k}^{2}\left(r_{k}\right)\right)} \\
& =(1+o(1)) e^{(2+o(1)) \eta_{k}(x)} .
\end{aligned}
$$

Applying elliptic estimates to 5.53, we conclude from (5.51, 5.54-5.56) that

$$
\eta_{k} \rightarrow \eta \quad \text { in } \quad C_{\mathrm{loc}}^{1}\left(\mathbb{R}^{2} \backslash\{0\}\right) \quad \text { as } \quad k \rightarrow \infty,
$$

where $\eta$ satisfies

$$
-\Delta_{\mathbb{R}^{2}} \eta=\alpha_{0} e^{2 \eta} \text { in } \quad \mathbb{R}^{2} \backslash\{0\} .
$$

For any $L>0,(5.57)$ together with (2.3), 5.52) and (5.55) leads to

$$
\begin{aligned}
\int_{\mathbb{B}_{L}(0) \backslash \mathbb{B}_{1 / L}(0)} e^{2 \eta} d x & =\lim _{k \rightarrow \infty} \int_{\mathbb{B}_{L}(0) \backslash \mathbb{B}_{1 / L}(0)} e^{2 \eta_{k}} d x \\
& =\lim _{k \rightarrow \infty} \int_{\mathbb{B}_{L}(0) \backslash \mathbb{B}_{1 / L}(0)} \frac{v_{k}(x) \widetilde{f_{k}}\left(\widetilde{x}_{i, k}+r_{k} x, v_{k}(x)\right)}{\varphi_{k}\left(r_{k}\right) \theta_{k}\left(r_{k}\right)} d x \\
& =\frac{1}{\alpha_{0}} \lim _{k \rightarrow \infty} \int_{\mathbb{B}_{L_{k}}\left(\widetilde{x}_{i, k}\right) \backslash \mathbb{B}_{r_{k} / L}\left(\widetilde{x}_{i, k}\right)} \widetilde{u}_{k}(y) \widetilde{f}_{k}\left(y, \widetilde{u}_{k}(y)\right) d y \\
& \leq \frac{C}{\alpha_{0}} .
\end{aligned}
$$

Letting $L \rightarrow \infty$, we have

$$
\int_{\mathbb{R}^{2}} e^{2 \eta} d x<\infty
$$

It follows from 5.26$),\left(\mathcal{H}_{\ell, 2}\right)$ and $\left(\mathcal{H}_{\ell, 4}\right)$ that

$$
\begin{aligned}
\int_{\left.\mathbb{B}_{s_{k}^{(\ell)}} \widetilde{x}_{i, k}\right)} \varphi_{k}\left(r_{k}\right) \widetilde{f}_{k}\left(y, \widetilde{u}_{k}(y)\right) d y \leq & \int_{\left.\mathbb{B}_{s_{k}^{(\ell)}}\left(\widetilde{x}_{i, k}\right) \backslash \mathbb{B}_{L r_{k}^{(\ell)}} \widetilde{x}_{i, k}\right)} \varphi_{k}(r) \widetilde{f}_{k}\left(y, \widetilde{u}_{k}(y)\right) d y \\
& +\frac{\varphi_{k}\left(s_{k}^{(\ell)}\right)}{\varphi_{k}\left(L r_{k}^{(\ell)}\right)} \int_{\mathbb{B}_{L r_{k}^{(\ell)}\left(\widetilde{x}_{i, k}\right)}} \varphi_{k}(r) \widetilde{f}_{k}\left(y, \widetilde{u}_{k}(y)\right) d y+o(1) \\
\leq & N_{k}\left(L r_{k}^{(\ell)}, s_{k}^{(\ell)}\right)+\frac{\varphi_{k}\left(s_{k}^{(\ell)}\right)}{\varphi_{k}\left(L r_{k}^{(\ell)}\right)} \Lambda_{k}\left(L r_{k}^{(\ell)}\right)+o(1) \\
& \rightarrow 0
\end{aligned}
$$

as $k \rightarrow \infty$ first then $L \rightarrow \infty$, that

$$
\int_{\mathbb{B}_{r_{k} / L}\left(\widetilde{x}_{i, k}\right)} \varphi_{k}\left(r_{k}\right) \widetilde{f_{k}}\left(y, \widetilde{u}_{k}(y)\right) d y \leq N_{k}\left(s_{k}^{(\ell)}, r_{k} / L\right)+o(1) \rightarrow 0
$$


as $k \rightarrow \infty$ first, then $L \rightarrow \infty$, and that

$$
\int_{\mathbb{B}_{r_{k} / L}\left(\widetilde{x}_{i, k}\right)} \varphi_{k}\left(r_{k}\right) \widetilde{u}_{k}(y) d y \leq \int_{\mathbb{B}_{r_{k} / L}\left(\widetilde{x}_{i, k}\right)} \widetilde{u}_{k}^{2}(y) d y+o(1) \rightarrow 0
$$

as $k \rightarrow \infty$. Therefore we conclude

$$
\begin{aligned}
\lim _{L \rightarrow \infty} \limsup _{k \rightarrow \infty}\left|\int_{\mathbb{B}_{1 / L}(0)}-\Delta \eta_{k} d x\right| \leq & \lim _{L \rightarrow \infty} \limsup _{k \rightarrow \infty} \int_{\left.\mathbb{B}_{r_{k} / L}\left(\widetilde{x}_{i, k}\right)\right)} \varphi_{k}\left(r_{k}\right) \widetilde{f}_{k}\left(y, \widetilde{u}_{k}(y)\right) d y \\
& +\lim _{L \rightarrow \infty} \limsup _{k \rightarrow \infty} \int_{\left.\mathbb{B}_{r_{k} / L}\left(\widetilde{x}_{i, k}\right)\right)} \varphi_{k}\left(r_{k}\right) \widetilde{\tau}_{k}(y) \widetilde{u}_{k}(y) d y \\
= & 0 .
\end{aligned}
$$

Let $\zeta_{k}$ be a sequence of solution to the equation

$$
\left\{\begin{array}{l}
-\Delta_{\mathbb{R}^{2}} \zeta_{k}(x)=e^{\psi_{i}\left(\widetilde{x}_{i, k}+r_{k} x\right)} \varphi_{k}\left(r_{k}\right) r_{k}^{2} \widetilde{f}_{k}\left(\widetilde{x}_{i, k}+r_{k} x, v_{k}(x)\right) \text { in } \mathbb{B}_{1}(0) \\
\zeta_{k}=\eta_{k} \text { on } \partial \mathbb{B}_{1}(0) .
\end{array}\right.
$$

Then in view of (5.53), $\eta_{k}-\zeta_{k}$ satisfies

$$
\left\{\begin{array}{l}
-\Delta_{\mathbb{R}^{2}}\left(\eta_{k}-\zeta_{k}\right)(x)=-e^{\psi_{i}\left(\widetilde{x}_{i, k}+r_{k} x\right)} \varphi_{k}\left(r_{k}\right) r_{k}^{2} \widetilde{\tau}_{k}\left(\widetilde{x}_{i, k}+r_{k} x\right) v_{k}(x) \text { in } \mathbb{B}_{1}(0) \\
\eta_{k}-\zeta_{k}=0 \quad \text { on } \partial \mathbb{B}_{1}(0)
\end{array}\right.
$$

Since $u_{k}$ is bounded in $L^{p}(\Sigma)$ for any $p>1$, applying elliptic estimates to (5.61), we get

$$
\left\|\eta_{k}-\zeta_{k}\right\|_{L^{\infty}\left(\mathbb{B}_{1}(0)\right)} \leq C
$$

for some constant $C$. By (5.57), $\eta_{k}$ is uniformly bounded on $\partial \mathbb{B}_{1}(0)$. In view of (5.60), the maximum principle implies that there exists some constant $C$ such that

$$
\zeta_{k}(x) \geq-C \text { for all } x \in \mathbb{B}_{1}(0) .
$$

Hence

$$
\eta_{k}(x) \geq-C \text { for all } x \in \mathbb{B}_{1}(0) .
$$

By (5.26), $\varphi_{k}\left(r_{k}\right) \leq v_{k}(x)+C$ for all $x \in \mathbb{B}_{1 / L}(0)$ and $L>1$. Note that

$$
\begin{aligned}
\varphi_{k}\left(r_{k}\right) r_{k}^{2} \widetilde{f}_{k}\left(\widetilde{x}_{i, k}+r_{k} x, v_{k}(x)\right) & =\varphi_{k}\left(r_{k}\right) r_{k}^{2} \theta_{k}\left(r_{k}\right) \frac{\widetilde{f_{k}}\left(\widetilde{x}_{i, k}+r_{k} x, v_{k}(x)\right)}{\theta_{k}\left(r_{k}\right)} \\
& =\left(\alpha_{0}+o(1)\right) e^{(1+o(1))\left(v_{k}^{2}(x)-\varphi_{k}^{2}\left(r_{k}\right)\right)} .
\end{aligned}
$$

Using the inequality $a^{2}-b^{2} \geq 2 b(a-b), a, b \geq 0$, we get $v_{k}^{2}(x)-\varphi_{i, k}^{2}\left(r_{k}\right) \geq 2 \eta_{k}(x)$ for all $x \in \mathbb{B}_{1}(0)$. Then (5.63) leads to

$$
\int_{\mathbb{B}_{1 / L}(0)} e^{\eta_{k}} d x \leq \frac{2}{\alpha_{0}} \int_{\mathbb{B}_{1 / L}(0)} \varphi_{k}\left(r_{k}\right) r_{k}^{2} \widetilde{f}_{k}\left(\widetilde{x}_{i, k}+r_{k} x, v_{k}(x)\right) d x
$$

for sufficiently large $k$. Combining (5.53), 5.59, (5.62) and 5.64), we obtain

$$
\lim _{L \rightarrow \infty} \lim _{k \rightarrow \infty} \int_{\substack{\mathbb{B}_{1 / L}(0) \\ 31}} \eta_{k} d x=0
$$


For any $\varphi \in C_{0}^{\infty}\left(\mathbb{R}^{2}\right)$, integration by parts gives

$$
\begin{aligned}
\int_{\mathbb{R}^{2}} \eta \Delta \varphi d x & =\lim _{L \rightarrow \infty} \int_{\mathbb{R}^{2} \backslash \mathbb{B}_{1 / L}(0)} \eta \Delta \varphi d x \\
& =\lim _{L \rightarrow \infty}\left(-\int_{\partial \mathbb{B}_{1 / L}(0)} \eta \partial_{\nu} \varphi d \sigma+\int_{\partial \mathbb{B}_{1 / L}(0)} \varphi \partial_{\nu} \eta d \sigma+\int_{\mathbb{R}^{2} \backslash \mathbb{B}_{1 / L}(0)} \varphi \Delta \eta d x\right) .
\end{aligned}
$$

It is clear that

$$
\begin{aligned}
\int_{\partial \mathbb{B}_{1 / L}(0)} \eta \partial_{\nu} \varphi d \sigma & =\lim _{k \rightarrow \infty} \int_{\partial \mathbb{B}_{1 / L}(0)} \eta_{k} \partial_{\nu} \varphi d \sigma \\
& =\lim _{k \rightarrow \infty}\left(\int_{\mathbb{B}_{1 / L}(0)} \eta_{k} \Delta \varphi d x+\int_{\mathbb{B}_{1 / L}(0)} \nabla \eta_{k} \nabla \varphi d x\right) \\
& =\lim _{k \rightarrow \infty}\left(\int_{\mathbb{B}_{1 / L}(0)} \eta_{k} \Delta \varphi d x+\int_{\partial \mathbb{B}_{1 / L}(0)} \varphi \partial_{v} \eta_{k} d \sigma-\int_{\mathbb{B}_{1 / L}(0)} \varphi \Delta \eta_{k} d x\right)
\end{aligned}
$$

Moreover, by Proposition 4.1 and (5.26), there exists some constant $C$ such that

$$
\left|\nabla \eta_{k}(x)\right|=\varphi_{k}\left(r_{k}\right) r_{k}\left|\nabla u_{k}\left(\widetilde{x}_{i, k}+r_{k} x\right)\right| \leq C /|x|
$$

for all $x \in \mathbb{B}_{1 / L}(0)$. This together with (5.59) leads to

$$
\begin{aligned}
\lim _{L \rightarrow \infty} \lim _{k \rightarrow \infty} \int_{\partial \mathbb{B}_{1 / L}(0)} \varphi \partial_{v} \eta_{k} d \sigma & =\varphi(0) \lim _{L \rightarrow \infty} \lim _{k \rightarrow \infty} \int_{\partial \mathbb{B}_{1 / L}(0)} \partial_{\nu} \eta_{k} d \sigma \\
& =\varphi(0) \lim _{L \rightarrow \infty} \lim _{k \rightarrow \infty} \int_{\mathbb{B}_{1 / L}(0)} \Delta \eta_{k} d x \\
& =0 .
\end{aligned}
$$

As a consequence

$$
\lim _{L \rightarrow \infty} \int_{\partial \mathbb{B}_{1 / L}(0)} \varphi \partial_{\nu} \eta d \sigma=\lim _{L \rightarrow \infty} \lim _{k \rightarrow \infty} \int_{\partial \mathbb{B}_{1 / L}(0)} \varphi \partial_{\nu} \eta_{k} d \sigma=0 .
$$

Inserting (5.58), (5.59), (5.65), (5.67) and (5.68) into (5.66), we obtain

$$
-\int_{\mathbb{R}^{2}} \eta \Delta \varphi d x=\lim _{L \rightarrow \infty} \int_{\mathbb{R}^{2} \mid \mathbb{B}_{1 / L}(0)} \alpha_{0} e^{2 \eta} \varphi d x=\int_{\mathbb{R}^{2}} \alpha_{0} e^{2 \eta} \varphi d x .
$$

Therefore $\eta$ is a distributional solution to the equation

$$
-\Delta_{\mathbb{R}^{2}} \eta=\alpha_{0} e^{2 \eta} \text { in } \mathbb{R}^{2} .
$$

By the regularity theory for elliptic equations, see for example ([4] , Chapter 2$), \eta \in C^{\infty}\left(\mathbb{R}^{2}\right)$. By a result of Chen-Li [5],

$$
\eta(x)=\log \frac{2}{1+|x|^{2}}-\log \sqrt{\alpha_{0}},
$$

and thus

$$
\int_{\mathbb{R}^{2}} e^{2 \eta} d x=\frac{4 \pi}{\alpha_{0}}
$$


This completes the proof of the lemma.

It follows from Lemma 5.6 that

$$
\lim _{L \rightarrow \infty} \lim _{k \rightarrow \infty} N_{k}\left(r_{k}^{(\ell+1)} / L, L r_{k}^{(\ell+1)}\right)=\alpha_{0} \int_{\mathbb{R}^{2}} e^{2 \eta^{(\ell+1)}} d x=4 \pi .
$$

This together with $(5.48)$ gives

$$
\lim _{L \rightarrow \infty} \lim _{k \rightarrow \infty} N_{k}\left(s_{k}^{(\ell)}, L r_{k}^{(\ell+1)}\right)=4 \pi
$$

By the inductive hypothesis $\left(\mathcal{H}_{\ell, 3}\right)$,

$$
\begin{aligned}
\lim _{L \rightarrow \infty} \lim _{k \rightarrow \infty} \Lambda_{k}\left(L r_{k}^{(\ell+1)}\right) & =\lim _{L \rightarrow \infty} \lim _{k \rightarrow \infty}\left(\Lambda_{k}\left(s_{k}^{(\ell)}\right)+N_{k}\left(s_{k}^{(\ell)}, L r_{k}^{(\ell+1)}\right)\right) \\
& =4 \pi(\ell+1) .
\end{aligned}
$$

Now we set $w_{k}^{(\ell+1)}(x)=\varphi_{k}\left(r_{k}^{(\ell+1)}\right)\left(\widetilde{u}_{k}(x)-\varphi_{k}\left(r_{k}^{(\ell+1)}\right)\right)$. Similar to Lemma 5.2, we have

Lemma 5.7 For any $\epsilon>0$, let $T_{k}^{(\ell+1)}=T_{k}^{(\ell+1)}(\epsilon)>r_{k}^{(\ell+1)}$ be the minimal number such that $\varphi_{k}\left(T_{k}^{(\ell+1)}\right)=\epsilon \varphi_{k}\left(r_{k}^{(\ell+1)}\right)$. Then $r_{k}^{(\ell+1)} / T_{k}^{(\ell+1)} \rightarrow 0$ as $k \rightarrow \infty$. Moreover, for any $b<2$ and sufficiently large $k, L$, there holds

$$
\overline{w_{k}^{(\ell+1)}}(r) \leq b \log \frac{r_{k}^{(\ell+1)}}{r}+C \text { for all } \quad L r_{k}^{(\ell+1)} \leq r \leq T_{k}^{(\ell+1)},
$$

where $C$ is a constant depending only on $\alpha_{0}$ and $(\Sigma, g)$, and we have

$$
\lim _{k \rightarrow \infty} N_{k}\left(s_{k}^{(\ell)}, T_{k}^{(\ell+1)}\right)=4 \pi .
$$

Proof. Since the proof is completely analogous to that of Lemma 4.2, except that instead of Proposition 3.1 we shall use Lemma 5.6, the details are omitted here.

For suitable $s_{k}^{(\ell+1)}=T_{k}^{(\ell+1)}\left(\epsilon_{k}\right)$, where $\epsilon_{k} \searrow 0$ is chosen such that $u_{k}\left(s_{k}^{(\ell+1)}\right) \rightarrow \infty$ as $k \rightarrow \infty$ and $r_{k}^{(\ell+1)} / s_{k}^{(\ell+1)} \rightarrow 0$ as $k \rightarrow \infty$. Moreover

$$
\lim _{k \rightarrow \infty} \Lambda_{k}\left(s_{k}^{(\ell+1)}\right)=4 \pi(\ell+1)
$$

and

$$
\lim _{L \rightarrow \infty} \lim _{k \rightarrow \infty} N_{k}\left(L r_{k}^{(\ell+1)}, s_{k}^{(\ell+1)}\right)=0
$$

By the definition of $s_{k}^{(\ell+1)}$,

$$
\lim _{k \rightarrow \infty} \frac{\varphi_{k}\left(s_{k}^{(\ell+1)}\right)}{\varphi_{k}\left(L r_{k}^{(\ell+1)}\right)}=0 \text { for any } L>0 .
$$

Hence $\left(\mathcal{H}_{\ell+1}\right)$ holds. This completes Step 2, and thus the proof of Proposition 5.1. 


\subsection{Quantization for non-simple blow-up points}

In this subsection, we shall prove a quantization result for non-simple blow-up points. We assume that $x_{i}^{*}$ is a non-simple blow-up point of order $m$, namely there exists a subset $\left\{i_{1}, \cdots, i_{m}\right\} \subset$ $\{1, \cdots, N\}$ such that $d_{g}\left(x_{i}^{*}, x_{\ell}^{*}\right)=0$ for all $\ell \in\left\{i_{1}, \cdots, i_{m}\right\}$ and $d_{g}\left(x_{j}^{*}, x_{i}^{*}\right)>0$ for all $j \in$ $\{1, \cdots, N\} \backslash\left\{i_{1}, \cdots, i_{m}\right\}$. In particular, $i \in\left\{i_{1}, \cdots, i_{m}\right\}$. Take an isothermal coordinate system $\left(U, \phi ;\left\{x^{1}, x^{2}\right\}\right)$ near $x_{i}^{*}$, where $U \subset \Sigma$ is a neighborhood of $x_{i}^{*}$ such that $x_{j}^{*} \notin \bar{U}$, the closure of $U$ for all $j \in\{1, \cdots, N\} \backslash\left\{i_{1}, \cdots, i_{m}\right\}, \phi: U \rightarrow \Omega \subset \mathbb{R}^{2}$ is a diffeomorphism with $\phi\left(x_{i}^{*}\right)=(0,0)$. We can find some $\delta>0$ such that $\mathbb{B}_{2 \delta}(0) \subset \Omega$. In this coordinate system, the metric $g=e^{\psi}\left(d x^{1^{2}}+d x_{2}{ }^{2}\right)$ for some smooth function $\psi: \Omega \rightarrow \mathbb{R}$ with $\psi(0,0)=0$. We shall prove the following result.

Proposition 5.8 Let $u_{k}, u_{\infty}, \tau_{k}, \tau_{\infty}, x_{i, k}$ and $x_{i}^{*}$ be as in Proposition 3.1. Suppose that $x_{i}^{*}$ is a non-simple blow-up point of order $m$ as above. Then up to a subsequence, there exists some positive integer I such that

$$
\lim _{k \rightarrow \infty} \int_{U}\left(\left|\nabla_{g} u_{k}\right|^{2}+\tau_{k} u_{k}^{2}\right) d v_{g}=\int_{U}\left(\left|\nabla_{g} u_{\infty}\right|^{2}+\tau_{\infty} u_{\infty}^{2}\right) d v_{g}+4 \pi I,
$$

where $U$ is a neighborhood of $x_{i}^{*}$ chosen as above.

Similarly as before we denote $\widetilde{x}_{j, k}=\phi\left(x_{j, k}\right)$ for $j \in\left\{i_{1}, \cdots, i_{m}\right\}, \widetilde{u}_{k}=u_{k} \circ \phi^{-1}, \widetilde{\tau}_{k}=\tau_{k} \circ \phi^{-1}$, and $\widetilde{f_{k}}\left(x, \widetilde{u}_{k}(x)\right)=f\left(\phi^{-1}(x), u_{k}\left(\phi^{-1}(x)\right)\right)$. Let $\varphi_{k}=\varphi_{k}^{(i)}, \Lambda_{k}=\Lambda_{k}^{(i)}$ and $N_{k}=N_{k}^{(i)}$ be as defined in (5.2), (5.3) and (5.4) respectively. The proof of Proposition 5.8 will be divided into several steps below.

Step 1. Blow-up analysis at the scale o $\left(\rho_{k}\right)$, where

$$
\rho_{k}=\rho_{k}^{(i)}=\frac{1}{2} \inf _{j \in\left\{i_{1}, \cdots, i_{m}\right\} \backslash\{i\}}\left|\widetilde{x}_{j, k}-\widetilde{x}_{i, k}\right| .
$$

By Proposition 3.1 we have $\lim _{L \rightarrow \infty} \lim _{k \rightarrow \infty} \Lambda_{k}\left(L r_{i, k}\right)=4 \pi$. Let $r_{k}^{(1)}=r_{i, k}$. We distinguish the following two cases to proceed.

Case 1 there exists some $0<\epsilon_{0}<1$ such that for all $t \in\left[r_{k}^{(1)}, \rho_{k}\right]$ there holds $\varphi_{k}(t) \geq \epsilon_{0} \varphi_{k}\left(r_{k}^{(1)}\right)$;

Case 2 for any $\epsilon>0$ there exists a minimal $T_{k}=T_{k}(\epsilon) \in\left[r_{k}^{(1)}, \rho_{k}\right]$ such that $\varphi_{k}\left(T_{k}\right)=\epsilon \varphi_{k}\left(r_{k}^{(1)}\right)$.

In Case 1, the decay estimate that we established in Lemma 5.2 remains valid on $\left[r_{k}^{(1)}, \rho_{k}\right]$. Moreover

$$
\lim _{L \rightarrow \infty} \lim _{k \rightarrow \infty} \Lambda_{k}\left(s_{k}\right)=4 \pi
$$

for any sequence $s_{k}$ satisfying $s_{k} / \rho_{k} \rightarrow 0$ and $s_{k} / r_{k}^{(1)} \rightarrow \infty$ as $k \rightarrow \infty$. The concentration analysis at scales up to $o\left(\rho_{k}\right)$ is complete.

In Case 2, as before we can find numbers $s_{k}^{(1)}<\rho_{k}$ with $\varphi_{k}\left(s_{k}^{(1)}\right) \rightarrow \infty$ as $k \rightarrow \infty, \Lambda_{k}\left(s_{k}^{(1)}\right) \rightarrow$ $4 \pi$ as $k \rightarrow \infty$, and $\varphi_{k}\left(s_{k}^{(1)}\right) / \varphi_{k}\left(L r_{k}^{(1)}\right) \rightarrow 0$ for any $L \geq 1$ as $k \rightarrow \infty$. We proceed by iteration up to some maximal index $\ell_{0} \geq 1$ where either Case 1 or (5.40) holds with final radii $r_{k}^{\left(\ell_{0}\right)}, s_{k}^{\left(\ell_{0}\right)}$, respectively. Hence

$$
\lim _{k \rightarrow \infty} \Lambda_{k}\left(s_{k}^{\left(\ell_{0}\right)}\right)=4 \pi \ell_{0}, \quad \lim _{k \rightarrow \infty} \varphi_{k}\left(s_{k}^{\left(\ell_{0}\right)}\right) / \varphi_{k}\left(L r_{k}^{\left(\ell_{0}\right)}\right)=0, \quad \forall L \geq 1
$$


and

$$
\lim _{k \rightarrow \infty} N_{k}\left(s_{k}^{\left(\ell_{0}\right)}, t_{k}\right)=0 \text { for any sequence } t_{k}=o\left(\rho_{k}\right)
$$

This leads to

$$
\lim _{L \rightarrow \infty} \lim _{k \rightarrow \infty} N_{k}\left(s_{k}^{\left(\ell_{0}\right)}, \rho_{k} / L\right)=0 .
$$

For otherwise, we can find some $\mu_{0}>0$ such that up to a subsequence

$$
\lim _{k \rightarrow \infty} N_{k}\left(s_{k}^{\left(\ell_{0}\right)}, \rho_{k}\right) \geq \lim _{k \rightarrow \infty} N_{k}\left(s_{k}^{\left(\ell_{0}\right)}, \rho_{k} / L\right) \geq \mu_{0}
$$

for all $L \geq 1$. Take $t_{k}^{\prime} \in\left(s_{k}^{\left(\ell_{0}\right)}, \rho_{k}\right)$ such that

$$
0<\lim _{k \rightarrow \infty} N_{k}\left(s_{k}^{\left(\ell_{0}\right)}, t_{k}^{\prime}\right)<\frac{1}{2 C_{0}},
$$

where $C_{0}$ is a constant as in Lemma 5.3. Then by Lemma 5.4 we have

$$
\lim _{L \rightarrow \infty} \lim _{k \rightarrow \infty} N_{k}\left(s_{k}^{\left(\ell_{0}\right)}, t_{k}^{\prime} / L\right)=0 .
$$

In view of (5.71) and (5.73), there exists some $v_{0}>0$ such that up to a subsequence, $t_{k}^{\prime} \geq v_{0} \rho_{k}$ for all $k$. This immediately implies (5.72) and completes Step 1.

To proceed, we introduce several terminologies concerning the classification of blow-up points near $x_{i}^{*}$. Define a set

$$
\mathcal{X}=\mathcal{X}^{(i)}=\left\{x_{i_{1}, k}, \cdots, x_{i_{m}, k}\right\}
$$

where each $x_{j, k}, j \in\left\{i_{1}, \cdots, i_{m}\right\}$, denotes a sequence $\left(x_{j, k}\right)$. In the sequel we do not distinguish sequences $\left(x_{j, k}\right)$ and points $x_{j, k}$. Let $t_{k}>0$ be a bounded sequence. For any $j \in\left\{i_{1}, \cdots, i_{m}\right\}$, we define a $t_{k}$-equivalent class associated to the sequence $x_{j, k}$ by

$$
\left[x_{j, k}\right]_{t_{k}}:=\left\{x_{\ell, k}: d_{g}\left(x_{\ell, k}, x_{j, k}\right)=o\left(t_{k}\right), \ell \in\left\{i_{1}, \cdots, i_{m}\right\}\right\} .
$$

The total number of sequences in $\left[x_{j, k}\right]_{t_{k}}$ is called the order of $\left[x_{j, k}\right]_{t_{k}}$. In particular, the order of $\left[x_{j, k}\right]_{\rho_{k}^{(j)}}$ is exactly one, while the order of $\left[x_{j, k}\right]_{\delta}$ is $m$. Actually we have $\left[x_{j, k}\right]_{\delta}=\mathcal{X}$. Moreover, if $x_{\ell, k} \in\left[x_{j, k}\right]_{t_{k}}$, then $x_{j, k} \in\left[x_{\ell, k}\right]_{t_{k}}$. Also, if $\left[x_{j, k}\right]_{t_{k}} \cap\left[x_{\ell, k}\right]_{t_{k}} \neq \varnothing$, then $\left[x_{j, k}\right]_{t_{k}}=\left[x_{\ell, k}\right]_{t_{k}}$. Hence every subset of $\mathcal{X}$ can be divided into several $t_{k}$-equivalent classes, any two of which have no intersection.

For any $1 \leq \ell<m$, we say that the property $\left(\mathcal{A}_{\ell}\right)$ holds for some $t_{k}$-equivalent class $\left[x_{j, k}\right]_{t_{k}}$ of order $\ell$, if either $(a)$ there exist $r_{k}>0$ and integer $I^{(j)}$ such that for some $\epsilon_{0}>0$ and all $t \in\left[r_{k}, t_{k}\right]$ there holds $\varphi_{k}^{(j)}(t) \geq \epsilon_{0} \varphi_{k}^{(j)}\left(r_{k}\right), \Lambda_{k}^{(j)}\left(L r_{k}\right) \rightarrow 4 \pi I^{(j)}$ and $N_{k}^{(j)}\left(L r_{k}, t_{k} / L\right) \rightarrow 0$ as $k \rightarrow \infty$ first, and then $L \rightarrow \infty$; or $(b)$ there exist sequences $r_{k}<s_{k}<t_{k}$ and an integer $I^{(j)}$ such that $\varphi_{k}^{(j)}\left(s_{k}\right) / \varphi_{k}^{(j)}\left(L r_{k}\right) \rightarrow 0$ as $k \rightarrow \infty$ for any $L \geq 1, \Lambda_{k}^{(j)}\left(t_{k} / L\right) \rightarrow 4 \pi I^{(j)}$ and $N_{k}^{(j)}\left(s_{k}, t_{k} / L\right) \rightarrow 0$ as $k \rightarrow \infty$ first, and then $L \rightarrow \infty$. While we say that the property $\left(\mathcal{A}_{m}\right)$ holds, if there exits some $j \in\left\{i_{1}, \cdots, i_{m}\right\}$ and integer $I^{(j)}$ such that $\Lambda_{k}^{(j)}(\delta / L) \rightarrow 4 \pi I^{(j)}$ as $k \rightarrow \infty$ first, and then $L \rightarrow \infty$.

According to Proposition 5.1, when $m=1,\left(\mathcal{A}_{1}\right)$ holds. When $m>1$, we let $\rho_{k, 0}=\rho_{k}$ and $\rho_{k, j}(1 \leq j \leq m-1)$ be defined as in (5.88) and (5.92) below. It follows from Step 1 that $\left(\mathcal{A}_{1}\right)$ holds for any $t_{k}$-equivalent class of order one, where

$$
t_{k} \in\left\{\rho_{k, 0}, \cdots, \rho_{k, m-1}\right\} .
$$


We now we make an induction procedure on both orders of $t_{k}$-equivalent class and $m$. Suppose that for some integer $v \geq 1$, when $m=v$, the property $\left(\mathcal{A}_{v}\right)$ holds; while when $m>v$, the property $\left(\mathcal{A}_{\ell}\right)$ holds for any $t_{k}$-equivalent class of order $1 \leq \ell \leq v$, where $t_{k}$ is as in (5.74). We shall prove the following: When $m=v+1$, the property $\left(\mathcal{A}_{v+1}\right)$ holds; When $m>v+1$, the property $\left(\mathcal{A}_{\ell}\right)$ holds for any $t_{k}$-equivalent class of order $1 \leq \ell \leq v+1$, where $t_{k}$ is as in (5.74). Assuming this induction argument is complete, we conclude that $\left(\mathcal{A}_{m}\right)$ holds for any integer $m$. It is easy to see that (5.69) follows immediately from $\left(\mathcal{A}_{m}\right)$ and the fact that $u_{k} \rightarrow u_{\infty}$ in $C_{\mathrm{loc}}^{1}\left(\Sigma \backslash\left\{x_{1}^{*}, \cdots, x_{i}^{*}\right\}\right)$.

In the next two steps, we shall prove that $\left(\mathcal{A}_{m}\right)$ holds for $m=v+1$. In Step 4, we shall prove that $\left(\mathcal{A}_{\ell}\right)$ holds for any $t_{k}$-equivalent class of order $1 \leq \ell \leq v+1$, where $t_{k}$ is as in 5.74).

Step 2. Blow-up analysis at the scale $\rho_{k}$.

Let $m=v+1$. Now we turn to carry out blow-up analysis at the scale $\rho_{k}$ near $\widetilde{x}_{i, k}$. We first assume that for some $L \geq 1$ there exists some sequence $\left(x_{k}\right)$ such that $\rho_{k} / L \leq R_{k}\left(x_{k}\right) \leq\left|x_{k}-\widetilde{x}_{i, k}\right| \leq$ $L \rho_{k}$ and

$$
\left|x_{k}-\widetilde{x}_{i, k}\right|^{2} \widetilde{u}_{k}\left(x_{k}\right) \widetilde{f}_{k}\left(x_{k}, \widetilde{u}_{k}\left(x_{k}\right)\right) \geq v_{0}>0 .
$$

By Proposition 4.1 we may assume that $\left|x_{k}-\widetilde{x}_{i, k}\right|=\rho_{k}$. The following estimate is important for our subsequent analysis.

Lemma 5.9 Assuming (5.75), we have $\varphi_{k}\left(\rho_{k}\right) / \varphi_{k}\left(r_{k}^{\left(\ell_{0}\right)}\right) \rightarrow 0$ as $k \rightarrow \infty$.

Proof. If we suppose that there exists some $\epsilon_{0}>0$ such that $\varphi_{k}\left(\rho_{k}\right) \geq \epsilon_{0} \varphi_{k}\left(r_{k}^{\left(\ell_{0}\right)}\right)$, then we set

$$
\left.w_{k}(x)=\varphi_{k}\left(r_{k}^{\left(\ell_{0}\right)}\right) \widetilde{u}_{k}(x)-\varphi_{k}\left(r_{k}^{\left(\ell_{0}\right)}\right)\right), \quad x \in \Omega .
$$

Similar to Lemma 5.2, there holds for any $b<2$

$$
\bar{w}_{k}(r) \leq b \log \frac{r_{k}^{\left(\ell_{0}\right)}}{r}+C
$$

for all $r \in\left[r_{k}^{\left(\ell_{0}\right)}, \rho_{k}\right]$. Let $\theta_{k}$ be as defined in (5.18). By (H5) and (iii) of Proposition 3.1, we find some uniform constant $C$ such that

$$
r_{k}^{\left(\ell_{0}\right)} \varphi_{k}\left(r_{k}^{\left(\ell_{0}\right)}\right) \theta_{k}\left(r_{k}^{\left(\ell_{0}\right)}\right) \leq C
$$

Hence we obtain

$$
\begin{aligned}
\left|x_{k}-\widetilde{x}_{i, k}\right|^{2} u_{k}\left(x_{k}\right) \widetilde{f}_{k}\left(x_{k}, \widetilde{u}_{k}\left(x_{k}\right)\right) & \leq C \rho_{k}^{2} \varphi_{k}\left(\rho_{k}\right) \theta_{k}\left(\rho_{k}\right) \\
& =C\left(r_{k}^{\left(\ell_{0}\right)}\right)^{2} \varphi_{k}\left(r_{k}^{\left(\ell_{0}\right)}\right) \theta_{k}\left(r_{k}^{\left(\ell_{0}\right)}\right)\left(\frac{\rho_{k}}{r_{k}^{\left(\ell_{0}\right)}}\right)^{2} \frac{\varphi_{k}\left(\rho_{k}\right)}{\varphi_{k}\left(r_{k}^{\left(\ell_{0}\right)}\right)} \frac{\theta_{k}\left(\rho_{k}\right)}{\theta_{k}\left(r_{k}^{\left(\ell_{0}\right)}\right)} \\
& \leq C\left(\rho_{k} / r_{k}^{\left(\ell_{0}\right)}\right)^{2} e^{(1+o(1))\left(\varphi_{k}^{2}\left(\rho_{k}\right)-\varphi_{k}^{2}\left(r_{k}^{\left(\ell_{0}\right)}\right)\right)} \\
& \leq C\left(\rho_{k} / r_{k}^{\left(\ell_{0}\right)}\right)^{2} e^{(1+o(1))\left(1+\epsilon_{0}\right) \bar{w}_{k}\left(\rho_{k}\right)} \\
& \leq C\left(\rho_{k} / r_{k}^{\left(\ell_{0}\right)}\right)^{2-(1+o(1))\left(1+\epsilon_{0}\right) b} \rightarrow 0
\end{aligned}
$$


as $k \rightarrow \infty$, if we choose $b<2$ such that $\left(1+\epsilon_{0}\right) b>2$. Here the first inequality follows from Proposition 4.1, the second one follows from (H4), (H5) and (5.77), while the third one is a consequence of our assumption $\varphi_{k}\left(\rho_{k}\right) \geq \epsilon_{0} \varphi_{k}\left(r_{k}^{\left(\ell_{0}\right)}\right)$, and the last one is implied by (5.76). The contradiction between (5.78) and (5.75) ends the proof of the lemma.

Lemma 5.9 implies that for any $\epsilon>0$ there exists $T_{k} \in\left[r_{k}^{\left(\ell_{0}\right)}, \rho_{k}\right]$ such that $\varphi_{k}\left(T_{k}\right)=\epsilon \varphi_{k}\left(r_{k}^{\left(\ell_{0}\right)}\right)$. Hence at scales up to order $o\left(\rho_{k}\right)$ we end up with (5.40), where $\ell$ is replaced by $\ell_{0}$. The desired quantization result at the scale $\rho_{k}$ then is a consequence of the following result.

Lemma 5.10 Assuming (5.75), then up to a subsequence we can find some $\alpha_{0} \geq v_{0}$ such that

$$
\lim _{k \rightarrow \infty}\left|x_{k}-\widetilde{x}_{i, k}\right|^{2} \widetilde{u}_{k}\left(x_{k}\right) \widetilde{f}_{k}\left(x_{k}, \widetilde{u}_{k}\left(x_{k}\right)\right)=\alpha_{0} .
$$

Moreover there exist a finite set $\mathcal{S}_{\infty} \subset \mathbb{R}^{2}$ such that

$$
\eta_{k}(x)=\widetilde{u}_{k}\left(x_{k}\right)\left(\widetilde{u}_{k}\left(\widetilde{x}_{i, k}+\rho_{k} x\right)-\widetilde{u}_{k}\left(x_{k}\right)\right) \rightarrow \eta(x)=\log \frac{2}{\sqrt{\alpha_{0}}\left(1+|x|^{2}\right)}
$$

in $C_{\mathrm{loc}}^{1}\left(\mathbb{R}^{2} \backslash \mathcal{S}_{\infty}\right)$ as $k \rightarrow \infty$.

Proof. It is obvious that (5.79) holds for some $\alpha_{0} \geq v_{0}>0$. Define

$$
v_{k}(y)=\widetilde{u}_{k}\left(\widetilde{x}_{i, k}+\rho_{k} y\right)
$$

for $y \in \Omega_{k}=\left\{y \in \mathbb{R}^{2}: \widetilde{x}_{i, k}+\rho_{k} y \in \Omega\right\}$. Let

$$
y_{j, k}=\frac{\widetilde{x}_{j, k}-\widetilde{x}_{i, k}}{\rho_{k}}
$$

and

$$
\mathcal{S}_{k}=\mathcal{S}_{k}^{(i)}=\left\{y_{j, k}: j=i_{1}, \cdots, i_{v+1}\right\} .
$$

Without loss of generality we assume either $\left|y_{j, k}\right| \rightarrow \infty$ or $y_{j, k} \rightarrow y_{j}, j=i_{1}, \cdots, i_{v+1}$, and we let $\mathcal{S}_{\infty}=\mathcal{S}_{\infty}^{(i)}$ be the set of accumulation points of $\mathcal{S}_{k}$. Also we let

$$
y_{0, k}=\frac{x_{k}-\tilde{x}_{i, k}}{\rho_{k}}
$$

be the scaled points of $x_{k}$ for which $\left(5.75\right.$ ) holds and which satisfy $\left|y_{0, k}\right|=1$. Moreover we can assume $y_{0, k} \rightarrow y_{0}$ as $k \rightarrow \infty$.

Since $\widetilde{u}_{k}\left(x_{k}\right) \rightarrow \infty$ by (5.75) and $\mathcal{S}_{\infty}$ is a finite set, we have by using Proposition 4.1 and a standard covering argument that

$$
v_{k}-\widetilde{u}_{k}\left(x_{k}\right) \rightarrow 0 \text { locally uniformly on } \mathbb{R}^{2} \backslash \mathcal{S}_{\infty}
$$

as $k \rightarrow \infty$. Using the same argument as in the proof of Lemma 5.6, we obtain

$$
\eta_{k} \rightarrow \eta \quad \text { in } \quad C_{\mathrm{loc}}^{1}\left(\mathbb{R}^{2} \backslash \mathcal{S}_{\infty}\right),
$$

where $\eta \in C^{\infty}\left(\mathbb{R}^{2} \backslash \mathcal{S}_{\infty}\right)$ satisfies the equation

$$
-\Delta_{\mathbb{R}^{2}} \eta=\alpha_{0} e^{2 \eta} \text { in } \quad \mathbb{R}^{2} \backslash \mathcal{S}_{\infty} .
$$


It follows from (5.80) that $v_{k} / \widetilde{u}_{k}\left(x_{k}\right) \rightarrow 1$ locally uniformly on $\mathbb{R}^{2} \backslash \mathcal{S}_{\infty}$. For any $L \geq 1$ we write

$$
K_{L}=\mathbb{B}_{L}(0) \backslash\left(\cup_{y_{j} \in \mathcal{S}_{\infty}} \mathbb{B}_{\delta / L}\left(y_{j}\right)\right) .
$$

Combining (H4), (H5), (2.3) and (5.80), we can estimate

$$
\begin{aligned}
\int_{\mathbb{R}^{2}} e^{2 \eta} d x & \leq \lim _{L \rightarrow \infty} \lim _{k \rightarrow \infty} \int_{K_{L}} \frac{v_{k}(x)}{\widetilde{u}_{k}\left(x_{k}\right)} e^{(1+o(1)) \eta_{k}\left(1+\frac{v_{k}(x)}{u_{k}\left(x_{k}\right)}\right)} d x \\
& =\lim _{L \rightarrow \infty} \lim _{k \rightarrow \infty} \int_{K_{L}} \frac{\widetilde{u}_{k}\left(\widetilde{x}_{i, k}+\rho_{k} x\right) \widetilde{f_{k}}\left(\widetilde{x}_{i, k}+\rho_{k} x, \widetilde{u}_{k}\left(\widetilde{x}_{i, k}+\rho_{k} x\right)\right)}{\widetilde{u}_{k}\left(x_{k}\right) \widetilde{f}_{k}\left(x_{k}, \widetilde{u}_{k}\left(x_{k}\right)\right)} d x \\
& \leq \frac{C}{v_{0}} \limsup _{k \rightarrow \infty} \int_{\Sigma} u_{k} f_{k}\left(x, u_{k}\right) d v_{g} \leq \frac{C}{v_{0}}
\end{aligned}
$$

Since $y_{j, k} \rightarrow y_{j}$ as $k \rightarrow \infty$, we can take sufficiently large $L$ and $k$ such that $\mathbb{B}_{1 / L}\left(y_{j}\right) \subset$ $\mathbb{B}_{2 / L}\left(y_{j, k}\right)$ and $\mathbb{B}_{2 / L}\left(y_{j, k}\right) \cap \mathbb{B}_{2 / L}\left(y_{\alpha, k}\right)=\varnothing$ for any $\alpha \neq j$. Moreover let $\ell$ be the order of the $\rho_{k}$-equivalent class $\left[x_{j, k}\right]_{\rho_{k}}$. Clearly $\ell \leq v$. By our inductive assumption, $\left(\mathcal{A}_{\ell}\right)$ holds for $\left[x_{j, k}\right]_{\rho_{k}}$. Noting that Lemma 5.9 excludes the possibility of Case 1 with $r_{k}^{(1)}$ replaced by $r_{k}^{\left(\ell_{0}\right)}$, we can find sequences $r_{k}^{(j)}<s_{k}^{(j)}$ such that

$$
\lim _{k \rightarrow \infty} \varphi_{k}\left(s_{k}^{(j)}\right) / \varphi_{k}\left(L r_{k}^{(j)}\right)=0, \quad \forall L \geq 1
$$

and

$$
\lim _{L \rightarrow \infty} \lim _{k \rightarrow \infty} N_{k}^{(j)}\left(s_{k}^{(j)}, \rho_{k} / L\right)=0,
$$

Note again that $y_{j, k} \rightarrow y_{j}$ as $k \rightarrow \infty$. There exists some constant $C$, which may depends on $\left|y_{j}\right|$ but not on $k$, such that $\left|\widetilde{x}_{j, k}-\widetilde{x}_{i, k}\right| \leq C \rho_{k}$. For any $x_{\alpha, k} \notin\left[x_{j, k}\right]_{\rho_{k}}$, we can take some large $L_{0}$ such that $\left|\widetilde{x}_{j, k}-\widetilde{x}_{\alpha, k}\right| \geq \rho_{k} /\left(2 L_{0}\right)$ for all sufficiently large $k$. Recalling that $\left|x_{k}-\widetilde{x}_{i, k}\right|=\rho_{k}$ and applying Proposition 4.1, we obtain

$$
\widetilde{u}_{k}\left(x_{k}\right) \leq \inf _{\partial \mathbb{B}_{2 \rho_{k} / L_{0}}\left(\widetilde{x}_{j, k}\right)} \widetilde{u}_{k}+C
$$

for some uniform constant $C$. As in the proof of Lemma 5.2, we can find another uniform constant $C$ such that for all $x \in \mathbb{B}_{2 \rho_{k} / L_{0}}\left(\widetilde{x}_{j, k}\right)$

$$
\widetilde{u}_{k}(x) \geq \inf _{\partial \mathbb{B}_{2 \rho_{k} / L_{0}}\left(\widetilde{x}_{j, k}\right)} \widetilde{u}_{k}-C .
$$

These two estimates immediately imply the existence of some uniform constant $C$ such that

$$
\widetilde{u}_{k}\left(x_{k}\right) \leq \widetilde{u}_{k}(x)+C \text { for all } x \in \mathbb{B}_{2 \rho_{k} / L}\left(\widetilde{x}_{j, k}\right),
$$

provided that $L \geq L_{0}$. Note that $g=e^{\psi}\left(d x^{1^{2}}+d x^{2^{2}}\right)$ for some smooth function $\psi$ with $\psi(0,0)=0$. 
By the equation (1.2), we have for large $L$

$$
\begin{aligned}
\int_{\mathbb{B}_{1 / L}\left(y_{j}\right)}\left|\Delta_{\mathbb{R}^{2}} \eta_{k}\right| d x \leq & \int_{\mathbb{B}_{1 / L}\left(y_{j}\right)} \rho_{k}^{2} \widetilde{u}_{k}\left(x_{k}\right) \widetilde{f}_{k}\left(\widetilde{x}_{i, k}+\rho_{k} x, v_{k}(x)\right) e^{\psi\left(\widetilde{x}_{i, k}+\rho_{k} x\right)} d x \\
& +\int_{\mathbb{B}_{1 / L}\left(y_{j}\right)} \rho_{k}^{2} \widetilde{u}_{k}\left(x_{k}\right) \widetilde{\tau}_{k}\left(\widetilde{x}_{i, k}+\rho_{k} x\right) v_{k}(x) e^{\psi\left(\widetilde{x}_{i, k}+\rho_{k} x\right)} d x \\
\leq & \int_{\mathbb{B}_{2 / L}\left(y_{j, k}\right)} \rho_{k}^{2} \widetilde{u}_{k}\left(x_{k}\right) \widetilde{f}_{k}\left(\widetilde{x}_{i, k}+\rho_{k} x, v_{k}(x)\right) e^{\psi\left(\widetilde{x}_{i, k}+\rho_{k} x\right)} d x \\
& +\int_{\mathbb{B}_{2 / L}\left(y_{j, k}\right)} \rho_{k}^{2} \widetilde{u}_{k}\left(x_{k}\right) \widetilde{\tau}_{k}\left(\widetilde{x}_{i, k}+\rho_{k} x\right) v_{k}(x) e^{\psi\left(\widetilde{x}_{i, k}+\rho_{k} x\right)} d x \\
= & \int_{\mathbb{B}_{2 \rho_{k} / L}\left(\widetilde{x}_{j, k}\right)} \widetilde{u}_{k}\left(x_{k}\right)\left(\widetilde{f}_{k}\left(y, \widetilde{u}_{k}(y)\right)+\widetilde{\tau}_{k}(y) \widetilde{u}_{k}(y)\right) e^{\psi(y)} d y .
\end{aligned}
$$

With the help of (5.81)-(5.83) and an obvious analogy to (5.26), we obtain

$$
\lim _{L \rightarrow \infty} \lim _{k \rightarrow \infty} \int_{\mathbb{B}_{1 / L}\left(y_{j}\right)}\left|\Delta_{\mathbb{R}^{2}} \eta_{k}\right| d x=0,
$$

analogous to (5.59). In the same way of proving (5.65) we get

$$
\lim _{L \rightarrow \infty} \lim _{k \rightarrow \infty} \int_{\mathbb{B}_{1 / L}\left(y_{j}\right)} \eta_{k} d x=0 .
$$

In view of (5.83), we can find some uniform constant $C$ such that for all $y \in \partial \mathbb{B}_{1 / L}\left(y_{j}\right)$

$$
\widetilde{u}_{k}\left(x_{k}\right) \widetilde{u}_{k}\left(\widetilde{x}_{j, k}+\rho_{k} y\right) \leq C,
$$

which together with Proposition 4.1 leads to

$$
\left|y-y_{j, k}\right|\left|\nabla_{\mathbb{R}^{2}} \eta_{k}(y)\right|=\left|\widetilde{x}_{i, k}+\rho_{k} x-\widetilde{x}_{j, k} \widetilde{u}_{k}\left(x_{k}\right)\right| \nabla_{\mathbb{R}^{2}} \widetilde{u}_{k}\left(\widetilde{x}_{i, k}+\rho_{k} x\right) \mid \leq C .
$$

This gives

$$
\left|\nabla_{\mathbb{R}^{2}} \eta_{k}(y)\right| \leq \frac{C}{\left|y-y_{j}\right|}
$$

for all $y \in \partial \mathbb{B}_{1 / L}\left(y_{j}\right)$, provided that $k$ is sufficiently large. Then we obtain an analogy to $(5.68)$, namely, for any $\varphi \in C_{0}^{\infty}\left(\mathbb{R}^{2}\right)$

$$
\lim _{L \rightarrow \infty} \int_{\partial \mathbb{B}_{1 / L}\left(y_{j}\right)} \varphi \partial_{\nu} \eta d \sigma=\lim _{L \rightarrow \infty} \int_{\partial \mathbb{B}_{1 / L}\left(y_{j}\right)} \eta \partial_{\nu} \varphi d \sigma=0 .
$$

This excludes $y_{j}$ as a singular point of $\eta$ as in Lemma 5.6. Since $y_{j}$ is any point of $\mathcal{S}_{\infty}$, we conclude that $\eta$ is a smooth solution to the equation

$$
-\Delta_{\mathbb{R}^{2}} \eta=\alpha_{0} e^{2 \eta} \text { in } \quad \mathbb{R}^{2} .
$$

The remaining part of the conclusions of the lemma follows from a result of Chen-Li [5]. 
Define a set

$$
A_{L, k}=\left\{x \in \Omega: \rho_{k} / L \leq R_{k}(x) \leq\left|x-\widetilde{x}_{i, k}\right| \leq L \rho_{k}\right\} .
$$

It follows from Proposition 4.1 that $u_{k}(x) / \widetilde{u}_{k}\left(x_{k}\right) \rightarrow 1$ uniformly in $A_{L, k}$ as $k \rightarrow \infty$. Thus by Lemma 5.10, in case of (5.75) there holds

$$
\begin{aligned}
\lim _{L \rightarrow \infty} \lim _{k \rightarrow \infty} \int_{A_{L, k}} \widetilde{u}_{k}(x) \widetilde{f}_{k}\left(x, \widetilde{u}_{k}(x)\right) d x & =\alpha_{0} \lim _{L \rightarrow \infty} \lim _{k \rightarrow \infty} \int_{A_{L, k}} \frac{\widetilde{u}_{k}(x) \widetilde{f}_{k}\left(x, \widetilde{u}_{k}(x)\right)}{\left.\widetilde{u}_{k}\right) \widetilde{f}_{k}\left(x_{k}, \widetilde{u}_{k}\left(x_{k}\right)\right)} d x \\
& =\alpha_{0} \int_{\mathbb{R}^{2}} e^{2 \eta(x)} d x \\
& =\alpha_{0} \frac{4 \pi}{\alpha_{0}}=4 \pi .
\end{aligned}
$$

Let

$$
X_{k, 1}=X_{k, 1}^{(i)}=\left\{\widetilde{x}_{j, k}: \exists C>0 \text { such that }\left|\widetilde{x}_{j, k}-\widetilde{x}_{i, k}\right| \leq C \rho_{k} \text { for all } k\right\} .
$$

We can divide $X_{k, 1}$ into several $\rho_{k}$-equivalent classes with their orders no more than $v$. Recalling our inductive assumption $\left(\mathcal{A}_{\ell}\right)$ with $1 \leq \ell \leq v$ and using (5.85), we can find some integer $I$ such that

$$
\lim _{L \rightarrow \infty} \lim _{k \rightarrow \infty} \Lambda_{k}\left(L \rho_{k}\right)=4 \pi(1+I) .
$$

On the other hand, if (5.75) does not hold, we have

$$
\lim _{L \rightarrow \infty} \lim _{k \rightarrow \infty} \int_{A_{L, k}} \widetilde{u}_{k} \widetilde{f}_{k}\left(x, \widetilde{u}_{k}\right) d x=0 .
$$

The energy estimate at the scale $\rho_{k}$ again is finished.

Step 3. Blow-up analysis at scales exceeding $\rho_{k}$.

Now we deal with blow-up analysis at scales exceeding $\rho_{k}$ near $\widetilde{x}_{i, k}$. Write

$$
X_{k, 0}=\left\{\widetilde{x}_{i_{1}, k}, \cdots, \widetilde{x}_{i_{m}, k}\right\} .
$$

Recalling (5.86), we let

$$
\rho_{k, 1}=\rho_{k, 1}^{(i)}=\left\{\begin{array}{lll}
\inf _{\tilde{x}_{j, k} \in X_{k, 0} \backslash X_{k, 1}} \frac{\left|\widetilde{x}_{j, k}-\widetilde{x}_{i, k}\right|}{2} & \text { if } & X_{k, 0} \backslash X_{k, 1} \neq \varnothing \\
\delta, & \text { if } & X_{k, 0} \backslash X_{k, 1}=\varnothing .
\end{array}\right.
$$

From this definition it follows that $\rho_{k, 1} / \rho_{k} \rightarrow \infty$ as $k \rightarrow \infty$. Then, using the obvious analogy of Lemma 5.4, either we have

$$
\lim _{L \rightarrow \infty} \lim _{k \rightarrow \infty} N_{k}\left(L \rho_{k}, \rho_{k, 1} / L\right)=0,
$$

and we iterate to the next scale; or there exist a sequence $t_{k}$ such that $t_{k} / \rho_{k} \rightarrow \infty, t_{k} / \rho_{k, 1} \rightarrow 0$ as $k \rightarrow \infty$ and up to a subsequence such that

$$
P_{k}\left(t_{k}\right) \geq v_{0}>0 \text { for all large } k .
$$

The argument then depends on whether (5.75) or (5.87) holds. In case of (5.75), as in Lemma 5.9, the bound (5.89) and Lemma 5.10 imply that $\varphi_{k}\left(t_{k}\right) / \varphi_{k}\left(\rho_{k}\right) \rightarrow 0$ as $k \rightarrow \infty$. Then we can 
argue as in (5.40) for $r \in\left[L \rho_{k}, \rho_{k, 1} / L\right]$ for sufficiently large $L$, and we can continue as before to resolve concentrations in this range of scales.

In case of (5.87) we further need to distinguish whether (5.40) or Case 1 holds at the final stage of our analysis at scales $o\left(\rho_{k}\right)$. Recalling that in case of (5.40) we have (5.70) and (5.72), in view of 5.87) for a suitable sequence of numbers $s_{k, 1}^{(0)}$ such that $s_{k, 1}^{(0)} / \rho_{k} \rightarrow \infty, t_{k} / s_{k, 1}^{(0)} \rightarrow \infty$ as $k \rightarrow \infty$ we obtain

$$
\lim _{L \rightarrow \infty} \lim _{k \rightarrow \infty}\left(\Lambda_{k}\left(s_{k, 1}^{(0)}\right)-\sum_{\widetilde{x}_{j, k} \in \widetilde{X}_{k, 1}} \Lambda_{k}^{(j)}\left(L r_{k}^{\left(\ell_{0}^{(j)}\right)}\right)\right)=0,
$$

where $\Lambda_{k}^{(j)}(r)$ and $r_{k}^{\left(e_{0}^{(j)}\right)}$ are computed as above with respect to the blow-up point $x_{j, k}$ and $\widetilde{X}_{k, 1}$ is the modular set containing all $t_{k}$-equivalent classes of $X_{k, 1}$, whence the distance between any two points of $\widetilde{X}_{k, 1}$ is greater than $\widetilde{v} \rho_{k}$ for some constant $\widetilde{v}>0$. In particular, with such a choice of $s_{k, 1}^{(0)}$ we find the immediate quantization result

$$
\lim _{k \rightarrow \infty} \Lambda_{k}\left(s_{k, 1}^{(0)}\right)=4 \pi I
$$

for some positive integer $I$. Here again we use the inductive assumption that $\left(\mathcal{A}_{l}\right)$ holds for all $\rho_{k}$-equivalent classes of order $\ell$ with $1 \leq \ell \leq v$. While in Case 1 if we assume there is some $\epsilon_{0}>0$ such that

$$
\varphi_{k}\left(s_{k, 1}^{(0)}\right) \geq \epsilon_{0} \varphi_{k}\left(\operatorname{Lr}_{k}^{\left(\ell_{0}^{(j)}\right)}\right)
$$

for all $r \in\left[L r_{k}^{\ell_{0}^{(j)}}, s_{k, 1}^{(0)}\right]$, then as before we have

$$
\lim _{L \rightarrow \infty} \lim _{k \rightarrow \infty} N_{k}^{(j)}\left(L r_{k}^{\left(\ell_{0}^{(j)}\right)}, s_{k, 1}^{(0)}\right)=0 .
$$

This contradicts (5.90) since $s_{k, 1}^{(0)} / \rho_{k} \rightarrow \infty$ as $k \rightarrow \infty$ and the modular set $\widetilde{X}_{k, 1}$ has at least two elements. This implies that (5.91) does not hold and up to a subsequence there holds for any $L \geq 1$

$$
\lim _{k \rightarrow \infty} \frac{\varphi_{k}\left(s_{k, 1}^{(0)}\right)}{\varphi_{k}\left(L r_{k}^{\left(\ell_{0}^{(j)}\right)}\right)}=0
$$

for all $x_{j, k} \in \widetilde{X}_{k, 1}$ where Case 1 holds. Then we can continue to resolve concentrations on the range $\left[s_{k, 1}^{(0)}, \rho_{k, 1} / L\right]$ as before.

We then proceed by iteration. For $\ell \geq 2$ we inductively define the sets

$$
X_{k, \ell}=X_{k, \ell}^{(i)}=\left\{\widetilde{x}_{j, k}: \exists C>0 \text { such that }\left|\widetilde{x}_{j, k}-\widetilde{x}_{i, k}\right| \leq C \rho_{k, \ell-1} \text { for all } k\right\}
$$

and let

$$
\rho_{k, \ell}=\rho_{k, \ell}^{(i)}=\left\{\begin{array}{lll}
\inf _{\tilde{x}_{j, k} \in X_{k, 0} \backslash X_{k, \ell}} \frac{\left|\widetilde{x}_{j, k}-\widetilde{x}_{i, k}\right|}{2} & \text { if } & X_{k, 0} \backslash X_{k, \ell} \neq \varnothing \\
\delta, & \text { if } & X_{k, 0} \backslash X_{k, \ell}=\varnothing .
\end{array}\right.
$$

Iteratively carrying out the above analysis at all scales $\rho_{k, \ell}$, exhausting all blow-up points $x_{j, k}$, up to a subsequence we obtain quantization result for $X_{k, 0}$. Then Step 3 is finished. 
It follows from Step 2 and Step 3 that there exists some integer $I$ such that

$$
\lim _{L \rightarrow \infty} \lim _{k \rightarrow \infty} \Lambda_{k}(\delta / L)=4 \pi I,
$$

different analogous to Lemma 5.5. Here and in the sequel, $I$ may denote different integer. Hence the property $\left(\mathcal{A}_{m}\right)$ holds when $m=v+1$.

Step 4. $\left(\mathcal{A}_{\ell}\right)$ holds for $1 \leq \ell \leq v+1$ when $m>v+1$.

When $m>v+1$, by our inductive assumption, $\left(\mathcal{A}_{\ell}\right)$ holds for all $1 \leq \ell \leq v$, it suffices to prove that $\left(\mathcal{A}_{v+1}\right)$ holds for any $t_{k}$-equivalent class $\left[x_{j, k}\right]_{t_{k}}$ of order $v+1$, where $j \in\left\{i_{1}, \cdots, i_{m}\right\}$ and $t_{k}$ is as in (5.74). This is completely analogous to that $\left(\mathcal{A}_{m}\right)$ holds in the case of $m=v+1$, which we proved above, except that (5.93) is replaced by

$$
\lim _{L \rightarrow \infty} \lim _{k \rightarrow \infty} \Lambda_{k}^{(j)}\left(t_{k} / L\right)=4 \pi I
$$

for some integer $I$. We omit the details here. This ends Step 4.

Proposition 5.8 follows from the property $\left(\mathcal{A}_{m}\right)$ and the last assertion of Proposition 3.1.

\section{Completion of the proof of Theorem 1.1}

In this section, we complete the proof of Theorem 1.1. Let $x_{i, k} \rightarrow x_{i}^{*}$ as $k \rightarrow \infty, 1 \leq i \leq N$, be as in Proposition 3.1. In view of possible non-simple blow-up points, without loss of generality, we may assume for some $q \leq N, x_{1}^{*}, \cdots, x_{q}^{*}$ are different from each other and $x_{\ell}^{*} \in\left\{x_{1}^{*}, \cdots, x_{q}^{*}\right\}$ for any $q+1 \leq \ell \leq N$. For any $1 \leq i \leq q$, we take an isothermal coordinate system $\left(U_{i}, \phi_{i} ;\left\{x^{1}, x^{2}\right\}\right)$ near $x_{i}^{*}$ such that $\phi_{i}\left(x_{i}^{*}\right)=(0,0)$ and $U_{i}=\phi_{i}^{-1}\left(\mathbb{B}_{\delta}(0)\right)$, where $\delta$ is chosen sufficiently small such that $\bar{U}_{i}$ does not contain any $x_{j}^{*}$ with $j \in\{1, \cdots, q\} \backslash\{i\}$. It follows from Propositions 5.1 and 5.8 that for some integer $I^{(i)}$ there holds

$$
\lim _{L \rightarrow \infty} \lim _{k \rightarrow \infty} \int_{\phi_{i}^{-1}\left(\mathbb{B}_{\delta / L}(0)\right)} u_{k} f_{k}\left(x, u_{k}\right) d v_{g}=4 \pi I^{(i)} .
$$

By Proposition 3.1, $u_{k} \rightarrow u_{\infty}$ in $C_{\mathrm{loc}}^{1}\left(\Sigma \backslash\left\{x_{1}^{*}, \cdots, x_{q}^{*}\right\}\right)$ as $k \rightarrow \infty$. hence

$$
\lim _{L \rightarrow \infty} \lim _{k \rightarrow \infty} \int_{\Sigma \backslash \cup_{i=1}^{q} \phi_{i}^{-1}\left(\mathbb{B}_{\delta / L}(0)\right)} u_{k} f_{k}\left(x, u_{k}\right) d v_{g}=\int_{\Sigma} u_{\infty} f_{k}\left(x, u_{\infty}\right) d v_{g}
$$

Combining these two estimates, we obtain

$$
\lim _{k \rightarrow \infty} \int_{\Sigma} u_{k} f_{k}\left(x, u_{k}\right) d v_{g}=\int_{\Sigma} u_{\infty} f_{k}\left(x, u_{\infty}\right) d v_{g}+4 \pi \sum_{i=1}^{q} I^{(i)} .
$$

This together with 1.2 leads to

$$
\lim _{k \rightarrow \infty} \int_{\Sigma}\left(\left|\nabla_{g} u_{k}\right|^{2}+\tau_{k} u_{k}^{2}\right) d v_{g}=\int_{\Sigma}\left(\left|\nabla_{g} u_{\infty}\right|^{2}+\tau_{\infty} u_{\infty}^{2}\right) d v_{g}+4 \pi \sum_{i=1}^{q} I^{(i)} .
$$


In view of (2.5), or particularly (2.4), we then have

$$
\lim _{k \rightarrow \infty} J_{k}\left(u_{k}\right)=J_{\infty}\left(u_{\infty}\right)+4 \pi \sum_{i=1}^{q} I^{(i)}
$$

This completes the proof of Theorem 1.1.

Acknowledgements. This work is supported by the NSFC 11171347. The author thanks the referee for his careful reading and valuable suggestions on the first version of this paper.

\section{References}

[1] Adimurthi, O. Druet, Blow-up analysis in dimension 2 and a sharp form of Trudinger-Moser inequality, Comm. Partial Differential Equations 29 (2004) 295-322.

[2] Adimurthi, M. Struwe, Global compactness properties of semilinear elliptic equation with critical exponential growth, J. Functional Analysis 175 (2000) 125-167.

[3] Adimurthi, Y. Yang, Multibubble analysis on $N$-Laplace equation in $\mathbb{R}^{N}$, Calc. Var. Partial Differential Equations 40 (2011) 1-14.

[4] T. Aubin, Nonlinear analysis on manifolds, Springer, 1982.

[5] W. Chen, C. Li, Classification of solutions of some nonlinear elliptic equations, Duke Math. J. 63 (1991) 615-622.

[6] O. Druet, Multibumps analysis in dimension 2: quantification of blow-up levels, Duke Math. J. 132 (2006) $217-269$.

[7] L. Fontana, Sharp borderline Sobolev inequalities on compact Riemannian manifolds, Comm. Math. Helv. 68 (1993) 415-454.

[8] T. Lamm, F. Robert, M. Struwe, The heat flow with a critical exponential nonlinearity, J. Functional Analysis 257 (2009) 2951-2998.

[9] L. Martinazzi, A threshold phenomenon for embeddings of $H_{0}^{m}$ into Orlicz spaces, Calc. Var. Partial Differential Equations 36 (2009) 493-506.

[10] L. Martinazzi, M. Struwe, Quantization for an elliptic equation of order $2 m$ with critical exponential non-linearity, Math Z. 270 (2012) 453-486

[11] J. Moser, A sharp form of an inequality by N.Trudinger, Ind. Univ. Math. J. 20 (1971) 1077-1091.

[12] S. Pohozaev, The Sobolev embedding in the special case $p l=n$, Proceedings of the technical scientific conference on advances of scientific reseach 1964-1965, Mathematics sections, 158-170, Moscov. Energet. Inst., Moscow, 1965.

[13] M. Struwe, Quantization for a fourth order equation with critical exponential growth, Math. Z. 256 (2007) $397-424$

[14] N. Trudinger, On embeddings into Orlicz spaces and some applications, J. Math. Mech. 17 (1967) 473-484.

[15] H. Wu, C. Shen, Y. Yu, Introduction to Riemannian geometry, Peking University Press, 1981.

[16] Y. Yang, L. Zhao, A class of Adams-Fontana type inequalities and related functionals on manifolds, Nonlinear Differ. Equ. Appl. 17 (2010) 119-135. 